\title{
An algebraic model for finite loop spaces
}

\author{
CARLES BROTO \\ RAN LEVI \\ BOB OLIVER
}

\begin{abstract}
A $p$-local compact group consists of a discrete $p$-toral group $S$, together with a fusion system and a linking system over $S$ which define a classifying space having very nice homotopy properties. We prove here that if some finite regular cover of a space $Y$ is the classifying space of a $p$-local compact group, then so is $Y_{p}^{\wedge}$. Together with earlier results by Dwyer and Wilkerson and by the authors, this implies as a special case that a finite loop space determines a $p$-local compact group at each prime $p$.
\end{abstract}

55R35; 20D20, 20E22

The theory of $p$-local compact groups, developed by the authors in [9], is designed to give a unified framework in which to study the $p$-local homotopy theory of classifying spaces of compact Lie groups and $p$-compact groups, as well as some other families of a similar nature. It also includes, and in many aspects generalizes, the earlier theory of $p$-local finite groups.

A finite loop space is a pair of spaces $(\Omega X, X)$, where $X$ is a connected CW complex and its loop space $\Omega X$ has the homotopy type of a finite CW complex. For example, for any compact Lie group $G$, the pair $(\Omega B G, B G)$ is a finite loop space, where $\Omega B G \simeq G$. A $p$-compact group, as defined by Dwyer and Wilkerson [12], is a $p$-local analog of a finite loop space. For each finite loop space $(\Omega X, X)$, the group of components of $\Omega X$ is finite, the component of the constant map $\Omega_{0} X$ has the homotopy type of $\Omega \tilde{X}$, where $\tilde{X}$ is the universal cover of $X$, and thus $(\Omega \tilde{X}, \tilde{X})$ is a connected finite loop space. Hence for each prime $p, p$-completion in the sense of Bousfield and Kan [4] yields a $p$-compact group $\left(\Omega \tilde{X}_{p}^{\wedge}, \tilde{X}_{p}^{\wedge}\right)$. Thus $\tilde{X}_{p}^{\wedge}$ is the classifying space of a $p$-local compact group: an algebraic object modelled on compact Lie groups and $p$-compact groups [9].

This motivated the following question: Let $B$ be a space with a finite regular covering $E \rightarrow B$, such that $E$ is the classifying space of a $p$-local compact group. Is $B$ itself the classifying space of a $p$-local compact group? Here, by a regular covering space, we mean one whose group of deck transformations acts transitively on fibres. 
Once we know that a $p$-complete space $X$ is the classifying space of a $p$-local compact group, several properties of $X$ then follow from our earlier work. For example, $X$ has a homotopy decomposition via classifying spaces of discrete $p-$ toral subgroups [9, Proposition 4.6], and the homotopy groups of the monoid of self homotopy equivalences of $X$ can be described in terms of automorphisms of the linking system associated to $X$ [9, Theorem 7.1]. The rational cohomology of $X$ is described in terms of the action of its "Weyl group" on the cohomology of its maximal torus (see the authors [10]), by analogy to the case of compact Lie groups. Also, using this linking system and results in Junod, the second author and Libman [15, Theorem A], we can construct certain "unstable Adams operations": self maps of $X$ characterized by their action on rational cohomology. It is these properties of the classifying spaces which provide our main motivation for studying and searching for examples of $p$-local compact groups.

Before stating our main theorem, we describe in a little more detail the objects of study. Let $\mathbb{Z} / p^{\infty}$ denote the union of all $\mathbb{Z} / p^{n}$ under the obvious inclusions. A discrete $p$-toral group is a group $S$ containing a normal subgroup of the form $\left(\mathbb{Z} / p^{\infty}\right)^{r}$ $(r \geq 0)$ with $p$-power index. A (saturated) fusion system over $S$ is a category whose objects are the subgroups of $S$, and whose morphisms are monomorphisms of groups which are required to satisfy certain axioms. We refer the reader to Section 1 (especially Definition 1.4 and Corollary 1.8) for details.

Given a saturated fusion system $\mathcal{F}$ over $S$, a centric linking system associated to $\mathcal{F}$ is a category $\mathcal{L}$ whose objects are those subgroups of $S$ which are $\mathcal{F}$-centric (Definition 1.5), and whose morphism sets are, in an appropriate sense, extensions of the corresponding morphism sets between the same objects in $\mathcal{F}$. This extra structure allows us to associate a "classifying space" to the fusion system in question. Thus a $p$-local compact group is a triple $(S, \mathcal{F}, \mathcal{L})$, where $S$ is a discrete $p$-toral group, $\mathcal{F}$ is a saturated fusion system over $S$, and $\mathcal{L}$ is a centric linking system associated to $\mathcal{F}$. The classifying space of a $p$-local compact group is the $p$-completion of the geometric realization of its linking system.

By [9, Theorem 10.7], the classifying space of each $p$-compact group is also the classifying space of a $p$-local compact group. However, for a compact Lie group $G$, $B G_{p}^{\wedge}$ is always the classifying space of a $p$-local compact group [9, Theorem 9.10], while the pair $\left(G_{p}^{\wedge}, B G_{p}^{\wedge}\right)$ is a $p$-compact group only when $\pi_{0}(G)$ is a $p$-group. Other examples of groups which give rise to $p$-local compact groups (but not necessarily $p$-compact groups) include semidirect products of $p$-completed tori by finite groups, and "torsion linear groups" (subgroups of $\mathrm{GL}_{n}(K)$ all of whose elements have finite order, where $\operatorname{char}(K) \neq p)$ [9, Theorem 8.10]. 
The main theorem in this paper says that if $X$ is the classifying space of a $p$-local compact group and $\pi$ is a finite group, then from any "extension" $Y$ of $X$ by $B \pi$ (ie $Y \rightarrow B \pi$ is a fibration with fibre $X$, or $X \rightarrow Y$ is a regular cover with group of deck transformations $\pi$ ), we can extract extensions of fusion and linking systems, and through that show that $Y_{p}^{\wedge}$ is the classifying space of a $p$-local compact group.

Theorem A Assume that $f: X \rightarrow Y$ is a finite regular covering space, where $X$ is homotopy equivalent to the classifying space of a $p$-local compact group. Then $Y_{p}^{\wedge}$ is also homotopy equivalent to the classifying space of a $p$-local compact group.

The proof of Theorem A (restated as Theorem 7.3) goes roughly as follows. Algebraic extensions of $p$-local finite groups were described and classified by the third author in [16], and this construction is generalized here (in Section 5) to the $p$-local compact case. In Section 6, we apply the theory of simplicial fibre bundles to construct an equivalence between the data needed to define an extension of a centric linking system $\mathcal{L}$ by a finite group $G$, and that needed to define a bundle over $B G$ with fibre the geometric realization $|\mathcal{L}|$ of $\mathcal{L}$. Finally, in Section 7, this equivalence is used to prove that any finite regular covering, as in the hypotheses of the theorem, can be realized by an extension of $p$-local compact groups as constructed in Section 5.

Special cases of Theorem A (those where the group of deck transformations is a $p-$ group or has order prime to $p$ ) are proven by Castellana, Grodal and the authors in [6]. But the arguments there cannot be applied to extensions by arbitrary finite groups, not even in the $p$-local finite case.

Let $p$ be a prime. A space $Y$ is $\mathbb{F}_{p}$-finite if $H_{*}\left(Y, \mathbb{F}_{p}\right)$ is a finite dimensional $\mathbb{F}_{p}-$ vector space. In [12], Dwyer and Wilkerson define a $p$-compact group to be an $\mathbb{F}_{p}$-finite loop space $\Omega X$ whose classifying space $X(=B(\Omega X))$ is $p$-complete. In [9], we show that every $p$-compact group $\Omega X$ gives rise to a $p$-local compact group $(S, \mathcal{F}, \mathcal{L})$ whose classifying space has the homotopy type of $X$.

Theorem B Let $X$ be any path connected space. Then for each prime $p$ such that $\Omega X$ is $\mathbb{F}_{p}$-finite, the space $X_{p}^{\wedge}$ has the homotopy type of the classifying space of a $p$-local compact group. In particular, this holds for each prime $p$ if $\Omega X$ has the homotopy type of a finite complex.

Proof Fix a prime $p$ such that $\Omega X$ is $\mathbb{F}_{p}$-finite. In particular, $H_{0}\left(\Omega X, \mathbb{F}_{p}\right)$ is finite, so $\pi_{1}(X)$ is a finite group, and hence $X_{p}^{\wedge}$ is $p$-complete by [4, Proposition I.5.2 and Section VII.5]. Note that if $\pi_{1}(X)$ is not a $p$-group, then $\Omega\left(X_{p}^{\wedge}\right)$ need not be equivalent to $(\Omega X)_{p}^{\wedge}$, and need not be $\mathbb{F}_{p}$-finite. 
Set $\pi=\pi_{1}(X)$, let $\tilde{X}$ be the universal cover of $X$ and consider the fibration

$$
\tilde{X} \rightarrow X \rightarrow B \pi \text {. }
$$

Then $\Omega \tilde{X}$ is a connected component of $\Omega X$, and hence is also $\mathbb{F}_{p}$-finite. Applying fibrewise $p$-completion, we obtain a fibration

$$
\tilde{X}_{p}^{\wedge} \rightarrow \bar{X} \rightarrow B \pi
$$

where $\tilde{X}_{p}^{\wedge}$ is $p$-complete and $\Omega \tilde{X}_{p}^{\wedge}$ is $\mathbb{F}_{p}$-finite. Thus $\tilde{X}_{p}^{\wedge}$ is the classifying space of a $p$-compact group. By [9, Theorem 10.7], $\tilde{X}_{p}^{\wedge}$ is the classifying space of a $p$-local compact group. So by Theorem $\mathrm{A}, \bar{X}_{p}^{\wedge} \simeq X_{p}^{\wedge}$ is the classifying space of a $p$-local compact group.

In particular, if $X$ is a path connected space such that $\Omega X$ has the homotopy type of a finite CW complex, then this holds for $X$ at all primes.

In the course of this work, we had to generalize several results already known for $p-$ local finite groups (or fusion systems over finite $p$-groups) to the $p$-local compact case.

- If $\mathcal{F}$ is a saturated fusion system over a discrete $p$-toral group $S$, then for each $Q \leq S$ and each $K \leq \operatorname{Aut}(Q)$ such that $Q$ is "fully $K$-normalized," the normalizer $N_{\mathcal{F}}^{K}(Q)$ is also a saturated fusion system (Definition 2.1 and Theorem 2.3).

- If $\mathcal{F}$ is a fusion system over a discrete $p$-toral group which is saturated after restriction to a certain family of subgroups $\mathcal{H}$, and certain other conditions are satisfied, then $\mathcal{F}$ is saturated (Theorem 4.2).

- A general theorem is shown for constructing extensions of $p$-local compact groups by finite groups, analogous to that in [16] (Theorem 5.4).

- We state and prove basic properties of transporter systems over discrete $p$-toral groups (Definition A.1) in the Appendix.

The proofs of these results have added considerably to the length of this paper, but we hope that they will also be useful in other future developments of the theory of $p$-local compact groups.

The paper is organized as follows. We start in Section 1 with the basic definitions and general background on $p$-local compact groups. Sections 2-5 consist mostly of the generalizations from the finite case to the $p$-local compact case just described. The equivalence between extensions of $\mathcal{L}$ by $G$ and bundles over $B G$ with fibre $|\mathcal{L}|$ is shown in Section 6. All of this is then combined in Section 7 to prove Theorem A. We end the paper with an Appendix where we collect the results we need on transporter systems over discrete $p$-toral groups. 
Notation When $G$ and $H$ are groups, $H<G$ always means that $H$ is a proper subgroup of $G$. Whenever $F: \mathcal{C} \rightarrow \mathcal{D}$ is a functor and $c, c^{\prime} \in \operatorname{Ob}(\mathcal{C})$, we write $F_{c, c^{\prime}}$ for the restriction of $F$ to $\operatorname{Mor}_{\mathcal{C}}\left(c, c^{\prime}\right)$, and write $F_{c}=F_{c, c}$.

The authors wish to thank the Universitat Autònoma de Barcelona, the Institute of Mathematics in Aberdeen and the University of Copenhagen for allowing the authors to meet and providing support at various times while this project was developing.

Acknowledgements Carles Broto is partially supported by FEDER-MICINN grant MTM 2010-20692. Ran Levi is partially supported by EPSRC grant EP/J014524/1. Bob Oliver is partially supported by UMR 7539 of the CNRS, and by project ANR BLAN08-2_338236, HGRT.

\section{Background on fusion and linking systems over discrete $p-$ toral groups}

In this section, we collect the definitions and some basic facts on $p$-local compact groups which will be useful throughout the paper. We refer to [9] for more details on many of the results described here.

Definition 1.1 A discrete $p$-torus is a group which is isomorphic to $\left(\mathbb{Z} / p^{\infty}\right)^{n}$ for some finite $n$. A discrete $p$-toral group is a group $P$ with a normal subgroup $P_{0} \unlhd P$ such that $P_{0}$ is a discrete $p$-torus and $P / P_{0}$ is a finite $p$-group. The subgroup $P_{0}$ will be called the identity component of $P$, and $P$ will be called connected if $P=P_{0}$. Set $\pi_{0}(P)=P / P_{0}$, and set $\operatorname{rk}(P)=n$ (the rank of $P$ ) if $P_{0} \cong\left(\mathbb{Z} / p^{\infty}\right)^{n}$. The order of $P$ is the pair $|P|:=\left(\operatorname{rk}(P),\left|\pi_{0}(P)\right|\right)$, regarded as an element of $\mathbb{N}^{2}$ ordered lexicographically.

Let $\operatorname{Sub}(S)$ be the set of all subgroups of a (discrete $p$-toral) group $S$. For any group $G$ and any $H, K \leq G, \operatorname{Hom}_{G}(H, K) \subseteq \operatorname{Hom}(H, K)$ denotes the set of homomorphisms induced by conjugation in $G$.

Definition 1.2 A fusion system $\mathcal{F}$ over a discrete $p$-toral group $S$ is a category with $\operatorname{Ob}(\mathcal{F})=\operatorname{Sub}(S)$, whose morphism sets $\operatorname{Hom}_{\mathcal{F}}(P, Q)$ satisfy the following conditions:

(a) $\operatorname{Hom}_{S}(P, Q) \subseteq \operatorname{Hom}_{\mathcal{F}}(P, Q) \subseteq \operatorname{Inj}(P, Q)$ for all $P, Q \in \operatorname{Sub}(S)$.

(b) Every morphism in $\mathcal{F}$ factors as an isomorphism in $\mathcal{F}$ followed by an inclusion.

With motivation from group theory, we make the following definition. 
Definition 1.3 Let $\mathcal{F}$ be a fusion system over a discrete $p$-toral group $S$. Two subgroups $P, Q \in \operatorname{Sub}(S)$ are $\mathcal{F}$-conjugate if they are isomorphic as objects of the category $\mathcal{F}$. Let $P^{\mathcal{F}}$ denote the set of all subgroups of $S$ which are $\mathcal{F}$-conjugate to $P$.

We are now ready to recall the definition of saturation of a fusion system.

Definition 1.4 Let $\mathcal{F}$ be a fusion system over a discrete $p$-toral group $S$.

- A subgroup $P \leq S$ is fully centralized in $\mathcal{F}$ if $\left|C_{S}(P)\right| \geq\left|C_{S}(Q)\right|$ for all $Q \in P^{\mathcal{F}}$.

- A subgroup $P \leq S$ is fully normalized in $\mathcal{F}$ if $\left|N_{S}(P)\right| \geq\left|N_{S}(Q)\right|$ for all $Q \in P^{\mathcal{F}}$.

- We say $\mathcal{F}$ is a saturated fusion system if the following three conditions hold:

(I) For each $P \leq S$ which is fully normalized in $\mathcal{F}, P$ is fully centralized in $\mathcal{F}, \operatorname{Out}_{\mathcal{F}}(P)$ is finite and $\operatorname{Out}_{S}(P) \in \operatorname{Syl}_{p}\left(\operatorname{Out}_{\mathcal{F}}(P)\right)$.

(II) If $P \leq S$ and $\varphi \in \operatorname{Hom}_{\mathcal{F}}(P, S)$ are such that $\varphi(P)$ is fully centralized, and if we set

$$
N_{\varphi}=\left\{g \in N_{S}(P) \mid{ }^{\varphi} c_{g} \in \operatorname{Aut}_{S}(\varphi(P))\right\},
$$

then there is $\bar{\varphi} \in \operatorname{Hom}_{\mathcal{F}}\left(N_{\varphi}, S\right)$ such that $\left.\bar{\varphi}\right|_{P}=\varphi$.

(III) If $P_{1} \leq P_{2} \leq P_{3} \leq \cdots$ is an increasing sequence of subgroups of $S$, with $P_{\infty}=\bigcup_{n=1}^{\infty} P_{n}$, and if $\varphi \in \operatorname{Hom}\left(P_{\infty}, S\right)$ is any homomorphism such that $\left.\varphi\right|_{P_{n}} \in \operatorname{Hom}_{\mathcal{F}}\left(P_{n}, S\right)$ for all $n$, then $\varphi \in \operatorname{Hom}_{\mathcal{F}}\left(P_{\infty}, S\right)$.

We next define certain classes of subgroups which play an important role in generating the morphisms in a fusion system.

Definition 1.5 Let $\mathcal{F}$ be a fusion system over a discrete $p$-toral group $S$. A subgroup $P \in \operatorname{Sub}(S)$ is $\mathcal{F}$-centric if $C_{S}(Q)=Z(Q)$ for all $Q \in P^{\mathcal{F}}$. A subgroup $P \in \operatorname{Sub}(S)$ is $\mathcal{F}$-radical if $\operatorname{Out}_{\mathcal{F}}(P):=\operatorname{Aut}_{\mathcal{F}}(P) / \operatorname{Inn}(P)$ is $p$-reduced, ie contains no nontrivial normal $p$-subgroup. Let $\mathcal{F}^{c}$ denote the full subcategory of $\mathcal{F}$ whose objects are the $\mathcal{F}$-centric subgroups of $S$.

In this paper it will be convenient to also use a different but equivalent definition of saturation, based on that due to Roberts and Shpectorov [18] in the finite case. We recall their definitions. 
Definition 1.6 Let $\mathcal{F}$ be a fusion system over a $p$-group $S$.

- A subgroup $P \leq S$ is fully automized in $\mathcal{F}$ if the index of $\operatorname{Aut}_{S}(P)$ in $\operatorname{Aut}_{\mathcal{F}}(P)$ is finite and prime to $p$.

- A subgroup $P \leq S$ is receptive in $\mathcal{F}$ if it has the following property: for each $Q \leq S$ and each $\varphi \in \operatorname{Iso}_{\mathcal{F}}(Q, P)$, if we set $N_{\varphi}=\left\{g \in N_{S}(Q) \mid{ }^{\varphi} c_{g} \in \operatorname{Aut}_{S}(P)\right\}$, then there is $\bar{\varphi} \in \operatorname{Hom}_{\mathcal{F}}\left(N_{\varphi}, S\right)$ such that $\left.\bar{\varphi}\right|_{P}=\varphi$.

In this terminology, axioms (I) and (II) in Definition 1.4 say that each fully normalized subgroup is fully centralized and fully automized, and each fully centralized subgroup is receptive.

By definition, if $P$ is fully automized in $\mathcal{F}$, then $\operatorname{Aut}_{\mathcal{F}}(P)$ is an extension of a discrete $p$-torus by a finite group. Hence it does contain maximal discrete $p$-toral subgroups, unique up to conjugation, which we regard as its Sylow $p$-subgroups. As usual, we let $\operatorname{Syl}_{p}\left(\operatorname{Aut}_{\mathcal{F}}(P)\right)$ denote the set of its Sylow $p$-subgroups.

The next lemma describes the relation between these concepts and those already defined.

Lemma 1.7 The following hold for any fusion system $\mathcal{F}$ over a discrete $p$-toral group $S$.

(a) Every receptive subgroup of $S$ is fully centralized.

(b) If $P \leq S$ is fully automized and receptive in $\mathcal{F}$, then it is fully normalized.

(c) If $P \leq S$ is fully automized and receptive in $\mathcal{F}$, and $Q \in P^{\mathcal{F}}$, then there is a morphism $\varphi \in \operatorname{Hom}_{\mathcal{F}}\left(N_{S}(Q), N_{S}(P)\right)$ such that $\varphi(Q)=P$.

Proof The proofs are identical to those given in [18] and Aschbacher, Kessar and the third author [2, Lemma I.2.6] in the finite case. A generalization of these statements will be proven in Lemma 2.2 below.

The following is an immediate consequence of Lemma 1.7.

Corollary 1.8 A fusion system $\mathcal{F}$ over a discrete $p$-toral group $S$ is saturated if and only if:

- Each subgroup of $S$ is $\mathcal{F}$-conjugate to one which is fully automized and receptive in $\mathcal{F}$.

- Axiom (III) holds for $\mathcal{F}$ : if $P_{1}<P_{2}<\cdots$ are subgroups of $S, P=\bigcup_{i=1} P_{i}$, and $\varphi \in \operatorname{Hom}(P, S)$ is such that $\left.\varphi\right|_{P_{i}} \in \operatorname{Hom}_{\mathcal{F}}\left(P_{i}, S\right)$ for each $i$, then $\varphi \in$ $\operatorname{Hom}_{\mathcal{F}}(P, S)$. 
When $G$ is a finite group and $\mathcal{H}$ is a set of subgroups of $G, \mathcal{T}_{\mathcal{H}}(G)$ denotes the $\mathcal{H}$-transporter category for $G: \operatorname{Ob}\left(\mathcal{T}_{\mathcal{H}}(G)\right)=\mathcal{H}$, and for $H, K \in \mathcal{H}$,

$$
\operatorname{Mor}_{\mathcal{T}_{\mathcal{H}}(G)}(H, K)=\left\{g \in G \mid{ }^{g} H \leq K\right\} .
$$

Definition 1.9 Let $\mathcal{F}$ be a fusion system over a discrete $p$-toral group $S$. Let $\mathcal{H}$ be a set of subgroups of $S$ which is closed under $\mathcal{F}$-conjugacy and overgroups, and includes all subgroups which are $\mathcal{F}$-centric and $\mathcal{F}$-radical. An $\mathcal{H}$-linking system associated to $\mathcal{F}$ is a category $\mathcal{L}$ with object set $\mathcal{H}$, together with a pair of functors

$$
\mathcal{T}_{\mathcal{H}}(S) \stackrel{\delta}{\longrightarrow} \mathcal{L} \stackrel{\pi}{\longrightarrow} \mathcal{F}
$$

such that each object is isomorphic (in $\mathcal{L}$ ) to one which is fully centralized in $\mathcal{F}$, and such that the following conditions are satisfied.

(A) The functor $\delta$ is the identity on objects, and $\pi$ is the inclusion on objects. For each $P, Q \in \mathcal{H}$ such that $P$ is fully centralized in $\mathcal{F}, C_{S}(P)$ acts freely on $\operatorname{Mor}_{\mathcal{L}}(P, Q)$ via $\delta_{P}$ and right composition, and $\pi_{P, Q}$ induces a bijection

$$
\operatorname{Mor}_{\mathcal{L}}(P, Q) / C_{S}(P) \stackrel{\cong}{\longrightarrow} \operatorname{Hom}_{\mathcal{F}}(P, Q) .
$$

(B) For each $P, Q \in \mathcal{H}$ and each $g \in N_{S}(P, Q), \pi_{P, Q}$ sends $\delta_{P, Q}(g) \in \operatorname{Mor}_{\mathcal{L}}(P, Q)$ to $c_{g} \in \operatorname{Hom}_{\mathcal{F}}(P, Q)$.

(C) For all $\psi \in \operatorname{Mor}_{\mathcal{L}}(P, Q)$ and all $g \in P, \psi \circ \delta_{P}(g)=\delta_{Q}(\pi(\psi)(g)) \circ \psi$.

A centric linking system is an $\mathrm{Ob}\left(\mathcal{F}^{c}\right)$-linking system, ie a linking system whose objects are the $\mathcal{F}$-centric subgroups of $S$.

When $P \leq Q$, we set $\iota_{P, Q}=\delta_{P, Q}(1)$. The morphisms $\iota_{P, Q}$ are regarded as the inclusions in $\mathcal{L}$.

Definition 1.10 A linking triple is a triple of the form $(S, \mathcal{F}, \mathcal{L})$, where $S$ is a discrete $p$-toral group, $\mathcal{F}$ is a saturated fusion system over $S$, and $\mathcal{L}$ is an $\mathcal{H}$-linking system for some family $\mathcal{H}$. A $p$-local compact group is a linking triple where $\mathcal{L}$ is a centric linking system.

Definition 1.11 Let $\mathcal{F}$ be a fusion system over a discrete $p$-toral group $S$, and let $\mathcal{H} \subseteq \operatorname{Sub}(S)$ be a family of subgroups. Then $\mathcal{H}$ is closed in $\operatorname{Sub}(S)$ if for each increasing sequence $P_{1}<P_{2}<P_{3}<\cdots$ of subgroups in $\mathcal{H}, \bigcup_{i=1} P_{i}$ is also in $\mathcal{H}$. 
Let $\mathcal{H} \subseteq \operatorname{Sub}(S)$ be a closed family. Then:

(a) $\mathcal{F}$ is $\mathcal{H}$-closed if for each sequence $P_{1}<P_{2}<\cdots$ in $\mathcal{H}$ with $P=\bigcup_{i=1} P_{i}$, and each homomorphism $\varphi \in \operatorname{Hom}(P, S)$ such that $\left.\varphi\right|_{P_{i}} \in \operatorname{Hom}_{\mathcal{F}}\left(P_{i}, S\right)$ for each $i, \varphi \in \operatorname{Hom}_{\mathcal{F}}(P, S)$.

(b) $\mathcal{F}$ is $\mathcal{H}$-generated if every morphism in $\mathcal{F}$ is a composite of restrictions of morphisms in $\mathcal{F}$ between subgroups in $\mathcal{H}$.

(c) $\mathcal{F}$ is $\mathcal{H}$-saturated if it is $\mathcal{H}$-closed and if every subgroup of $\mathcal{H}$ is $\mathcal{F}$-conjugate to a subgroup which is fully automized and receptive.

The following two results, both generalizations to discrete $p$-toral groups of wellknown properties of $p$-groups, will be useful.

Lemma 1.12 [9, Lemma 1.8] If $P<Q$ are discrete $p$-toral groups, $P<N_{Q}(P)$.

Lemma 1.13 Let $Q \unlhd P$ be discrete $p$-toral groups, where $|P / Q|<\infty$. Then the group

$$
\left\{\alpha \in \operatorname{Aut}(P)|\alpha|_{Q}=\mathrm{Id},[\alpha, P] \leq Q\right\}
$$

is discrete $p$-toral.

Proof Let $A$ denote this group of automorphisms. For each $\alpha \in A$ and each $g \in P$, $\alpha(g)=g \chi(g)$ for some $\chi(g) \in Q$ since $[\alpha, P] \leq Q, c_{g}=c_{\alpha(g)} \in \operatorname{Aut}(Q)$ since $\left.\alpha\right|_{Q}=\mathrm{Id}$, and hence $\chi(g) \in Z(Q)$. Also, for $a \in Q, \alpha(g a)=g \chi(g) a=(g a) \chi(g)$ since $\alpha(a)=a$ and $[a, \chi(g)]=1$, so $\chi(g a)=\chi(g)$. Thus $\alpha$ is determined by the map $\chi: P / Q \rightarrow Z(Q)$. The resulting injection $A \rightarrow \operatorname{Map}(P / Q, Z(Q))$ is a homomorphism, and so $A$ is discrete $p$-toral since $\operatorname{Map}(P / Q, Z(Q)) \cong Z(Q)^{|P / Q|}$ is so.

\section{Normalizer fusion subsystems}

Let $\mathcal{F}$ be a fusion system over a discrete $p$-toral group $S$. For each $Q \leq S$ and each $K \leq \operatorname{Aut}(Q)$, define

$$
\begin{aligned}
\operatorname{Aut}_{\mathcal{F}}^{K}(Q) & =K \cap \operatorname{Aut}_{\mathcal{F}}(Q), \\
\operatorname{Aut}_{S}^{K}(Q) & =K \cap \operatorname{Aut}_{S}(Q), \\
N_{S}^{K}(Q) & =\left\{x \in N_{S}(Q) \mid c_{x} \in K\right\} \quad \text { (the } K \text {-normalizer of } Q \text { in } S \text { ). }
\end{aligned}
$$


Definition 2.1 Let $\mathcal{F}$ be a fusion system over a discrete $p$-toral group $S$. Fix a subgroup $Q \leq S$ and a group of automorphisms $K \leq \operatorname{Aut}(Q)$. Then:

- $Q$ is fully $K$-automized in $\mathcal{F}$ if $\operatorname{Aut}_{S}^{K}(Q) \in \operatorname{Syl}_{p}\left(\operatorname{Aut}_{\mathcal{F}}^{K}(Q)\right)$.

- $Q$ is fully $K$-normalized in $\mathcal{F}$ if for each $\varphi \in \operatorname{Hom}_{\mathcal{F}}(Q, S)$,

$$
\left|N_{S}^{K}(Q)\right| \geq\left|N_{S}^{\varphi} K(\varphi(Q))\right|,
$$

where ${ }^{\varphi} K=\left\{\varphi \alpha \varphi^{-1} \mid \alpha \in K\right\} \leq \operatorname{Aut}(\varphi(Q))$.

- $N_{\mathcal{F}}^{K}(Q) \subseteq \mathcal{F}$ is the fusion system over $N_{S}^{K}(Q)$ where for $P, R \leq N_{S}^{K}(Q)$,

$\operatorname{Hom}_{N_{\mathcal{F}}^{K}(Q)}(P, R)=\left\{\varphi \in \operatorname{Hom}_{\mathcal{F}}(P, R) \mid\right.$ there exists $\bar{\varphi} \in \operatorname{Hom}_{\mathcal{F}}(P Q, R Q)$

$$
\text { with } \left.\left.\bar{\varphi}\right|_{P}=\varphi, \bar{\varphi}(Q)=Q \text { and }\left.\bar{\varphi}\right|_{Q} \in K\right\} \text {. }
$$

As special cases of the above definition, we set $N_{\mathcal{F}}(Q)=N_{\mathcal{F}}^{\operatorname{Aut}(Q)}(Q)$ and set $C_{\mathcal{F}}(Q)=N_{\mathcal{F}}^{\{1\}}(Q)$ : the normalizer and centralizer fusion systems, respectively, of $Q$.

The next lemma is a generalization of results in [18] to fusion systems over discrete $p$-toral groups.

Lemma 2.2 Let $\mathcal{F}$ be a fusion system over a discrete $p$-toral group $S$, and let $\mathcal{P}$ be an $\mathcal{F}$-conjugacy class of subgroups of $S$. Assume either that $\mathcal{F}$ is saturated, or (more generally) that $\mathcal{P}$ contains a subgroup which is fully automized and receptive in $\mathcal{F}$. Then the following hold for each $P \in \mathcal{P}$ and each $K \leq \operatorname{Aut}(P)$.

(a) The subgroup $P$ is fully centralized if and only if it is receptive.

(b) The subgroup $P$ is fully $K$-normalized if and only if it is fully $K$-automized and receptive. In this case, for each $Q \in \mathcal{P}$ and each $\varphi \in \operatorname{Iso}_{\mathcal{F}}(Q, P)$, there are $\chi \in \operatorname{Aut}_{\mathcal{F}}^{K}(P)$ and $\bar{\varphi} \in \operatorname{Hom}_{\mathcal{F}}\left(N_{S}^{K^{\varphi}}(Q) \cdot Q, N_{S}^{K}(P) \cdot P\right)$ such that $\left.\bar{\varphi}\right|_{Q}=\chi \circ \varphi$.

Proof (a) For fusion systems over finite $p$-groups, this is shown in [18, Propositions 3.7 and 4.6] and in [2, Lemma I.2.6(c)]. Those proofs carry over unchanged to the discrete $p$-toral case.

(b) Assume that $P$ is fully $K$-automized and receptive in $\mathcal{F}$. Fix $Q \in \mathcal{P}$ and $\varphi \in \operatorname{Iso}_{\mathcal{F}}(Q, P)$. Then ${ }^{\varphi} \operatorname{Aut}_{S}^{K^{\varphi}}(Q)$ is a discrete $p$-toral subgroup of $\operatorname{Aut}_{\mathcal{F}}^{K}(P)$, and since $P$ is fully $K$-automized, there is $\chi \in \operatorname{Aut}_{\mathcal{F}}^{K}(P)$ such that ${ }^{\varphi} \operatorname{Aut}_{S}^{K^{\varphi}}(Q) \leq$ $\operatorname{Aut}_{S}^{K}(P)$. Note that $K^{\varphi}=K^{\chi \varphi}$ since $\chi \in K$.

Set $\psi=\chi \circ \varphi$. Then ${ }^{\psi} \operatorname{Aut}_{S}^{K}{ }^{\psi}(Q) \leq \operatorname{Aut}_{S}^{K}(P)$, and since $P$ is receptive, there is a morphism

$$
\bar{\psi} \in \operatorname{Hom}_{\mathcal{F}}\left(N_{S}^{K^{\psi}}(Q) \cdot Q, N_{S}^{K}(P) \cdot P\right)
$$


which extends $\psi$. In particular, $\left|N_{S}^{K^{\varphi}}(Q)\right|=\left|N_{S}^{K^{\psi}}(Q)\right| \leq\left|N_{S}^{K}(P)\right|$. Since $Q$ and $\varphi$ were arbitrary, this proves that $P$ is fully $K$-normalized in $\mathcal{F}$.

Now assume $P$ is fully $K$-normalized. Fix $R \in \mathcal{P}$ and $\psi \in \operatorname{Iso}_{\mathcal{F}}(P, R)$ such that $R$ is fully automized and receptive in $\mathcal{F}$. Fix $T \in \operatorname{Syl}_{p}\left(\operatorname{Aut}_{\mathcal{F}}^{\psi} K(R)\right)$ such that $T \geq$ ${ }^{\psi}$ Aut $_{S}^{K}(P)$. Since $R$ is fully automized, there is $\alpha \in \operatorname{Aut}_{\mathcal{F}}(R)$ such that ${ }^{\alpha} T \leq \operatorname{Aut}_{S}(R)$. Set $\varphi=\alpha \circ \psi$. Then ${ }^{\varphi} \operatorname{Aut}_{S}^{K}(P) \leq{ }^{\alpha} T \leq \operatorname{Aut}_{S}(R)$, and since $R$ is receptive, $\varphi$ extends to $\bar{\varphi} \in \operatorname{Hom}_{\mathcal{F}}\left(N_{S}^{K}(P) \cdot P, N_{S}(R)\right)$, where $\operatorname{Im}(\bar{\varphi}) \leq N_{S}^{\varphi} K(R)$.

Consider the commutative diagram

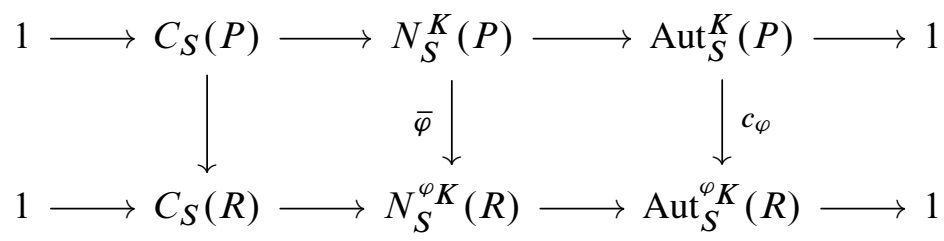

where the rows are exact and all vertical maps are monomorphisms. Since $P$ is fully $K$-normalized, $\bar{\varphi}\left(N_{S}^{K}(P)\right)=N_{S}^{\varphi}(R)$, and hence $\bar{\varphi}\left(C_{S}(P)\right)=C_{S}(R)$ and ${ }^{\varphi} \operatorname{Aut}_{S}^{K}(P)=\operatorname{Aut}_{S}^{\varphi} K^{K}(R)$. Thus $P$ is receptive by (a) (and since $R$ is receptive).

Now, $T \in \operatorname{Syl}_{p}\left(\operatorname{Aut}_{\mathcal{F}}^{\psi} K(R)\right)$ by assumption, so ${ }^{\alpha} T \in \operatorname{Syl}_{p}\left(\operatorname{Aut}_{\mathcal{F}}{ }^{\prime}(R)\right)$. Also, ${ }^{\alpha} T \leq$ $\operatorname{Aut}_{S}(R)$, and hence ${ }^{\alpha} T=\operatorname{Aut}_{S}^{\varphi} K(R)$. Thus $\operatorname{Aut}_{S}^{K}(P) \in \operatorname{Syl}_{p}\left(\operatorname{Aut}_{\mathcal{F}}^{K}(P)\right)$, so $P$ is fully $K$-automized, and this finishes the proof of (b).

The main result in this section is that normalizer fusion subsystems over discrete $p$-toral groups are saturated. The proof given here is modelled on that of [2, Theorem I.5.5].

Theorem 2.3 Fix a saturated fusion system $\mathcal{F}$ over a discrete $p$-toral group $S$. Assume $Q \leq S$ and $K \leq \operatorname{Aut}(Q)$ are such that $Q$ is fully $K$-normalized in $\mathcal{F}$. Then $N_{\mathcal{F}}^{K}(Q)$ is a saturated fusion system over $N_{S}^{K}(Q)$.

Proof Set $S_{+}=N_{S}^{K}(Q)$ and $\mathcal{F}_{+}=N_{\mathcal{F}}^{K}(Q)$ for short. For each $P \leq S_{+}$, set

$$
K_{P}=\left\{\alpha \in \operatorname{Aut}(P Q)|\alpha(P)=P, \alpha(Q)=Q, \alpha|_{Q} \in K\right\} .
$$

We need to show the following statements.

(a) Each subgroup of $S_{+}$is $\mathcal{F}_{+}-$conjugate to a subgroup $P$ such that $P Q$ is fully $K_{P}$-normalized in $\mathcal{F}$.

(b) If $P \leq S_{+}$and $P Q$ is fully $K_{P}$-normalized in $\mathcal{F}$, then $P$ is fully automized in $\mathcal{F}_{+}$.

(c) If $P \leq S_{+}$and $P Q$ is fully $K_{P}$-normalized in $\mathcal{F}$, then $P$ is receptive in $\mathcal{F}_{+}$.

(d) Axiom (III) holds for $N_{\mathcal{F}}^{K}(Q)$. 
The theorem will then follow immediately from Corollary 1.8 .

The proofs of (a), (b), and (c) are identical to the corresponding proofs in [2, Theorem I.5.5] (which is stated for fusion systems over finite $p$-groups). It remains to prove (d).

Fix subgroups $P_{1} \leq P_{2} \leq P_{3} \leq \cdots \leq S_{+}$, and set $P=\bigcup_{i=1}^{\infty} P_{i}$. Assume $\varphi \in$ $\operatorname{Hom}\left(P, S_{+}\right)$is such that $\varphi_{i}:=\left.\varphi\right|_{P_{i}} \in \operatorname{Hom}_{\mathcal{F}_{+}}\left(P_{i}, S_{+}\right)$for each $i$.

For each $i$, set

$$
X_{i}=\left\{\psi \in \operatorname{Hom}_{\mathcal{F}}\left(P_{i} Q, S\right)|\psi|_{P_{i}}=\varphi_{i}, \psi(Q)=Q,\left.\psi\right|_{Q} \in K\right\} \neq \varnothing,
$$

and let $\bar{X}_{i}$ be the image of $X_{i}$ in $\operatorname{Rep}_{\mathcal{F}}\left(P_{i} Q, S\right)$ (equivalently, the set of $C_{S}\left(P_{i}\right)$-conjugacy classes of morphisms in $\left.X_{i}\right)$. Since $\operatorname{Rep}_{\mathcal{F}}\left(P_{i} Q, S\right)$ is finite by [9, Lemma 2.5], so is $\bar{X}_{i}$. There are natural restriction maps $\bar{X}_{i} \rightarrow \bar{X}_{i-1}$, and since the sets are finite and nonempty, the inverse limit is nonempty. Fix an element $\left(\left[\psi_{i}\right]\right)_{i=1}^{\infty}$ in the inverse limit. Thus $\psi_{i} \in \operatorname{Hom}_{\mathcal{F}}\left(P_{i} Q, S\right),\left.\psi_{i}\right|_{P_{i}}=\varphi_{i},\left.\psi_{i}\right|_{Q} \in K$, and $\left.c_{g_{i-1}} \circ \psi_{i}\right|_{P_{i-1}} Q=\psi_{i-1}$ for some $g_{i-1} \in C_{S}\left(P_{i-1}\right)$.

Since $S$ is Artinian (cf [9, Proposition 1.2]), there is $N$ such that $C_{S}\left(P_{i}\right)=C_{S}(P)$ for each $i \geq N$. For each $i \geq N$, set $\psi_{i}^{\prime}=c_{g_{N}} \circ c_{g_{N+1}} \circ \cdots \circ c_{g_{i-1}} \circ \psi_{i}$. Then $\left.\psi_{i}^{\prime}\right|_{P_{i-1}}=\psi_{i-1}^{\prime}$, and $\left.\psi_{i}^{\prime}\right|_{P_{i}}=\varphi_{i}$. Set $\psi^{\prime}=\bigcup_{i=1}^{\infty} \psi_{i}^{\prime}$. Then $\psi^{\prime} \in \operatorname{Hom}_{\mathcal{F}}(P Q, S)$ by axiom (III) for $\mathcal{F},\left.\psi^{\prime}\right|_{P}=\varphi,\left.\psi^{\prime}\right|_{Q}=\left.\psi_{N}\right|_{Q} \in K$, and so $\varphi \in \operatorname{Hom}_{\mathcal{F}_{+}}(P, S)$.

The following is one easy application of Theorem 2.3.

Lemma 2.4 Let $\mathcal{F}$ be a saturated fusion system over a discrete $p$-toral group $S$. Assume $Q \unlhd P \leq S$, where $Q$ is $\mathcal{F}$-centric. Let $\varphi, \varphi^{\prime} \in \operatorname{Hom}_{\mathcal{F}}(P, S)$ be such that $\left.\varphi\right|_{Q}=\left.\varphi^{\prime}\right|_{Q}$. Then there is $x \in Z(Q)$ such that $\varphi^{\prime}=\varphi \circ c_{x}$.

Proof Since $\varphi \circ c_{g}=c_{\varphi(g)} \circ \varphi$ for each $g \in Q$, it suffices to show that $\varphi^{\prime}=c_{y} \circ \varphi$ for some $y \in Z(\varphi(Q))$. Upon replacing $P$ by $\varphi^{\prime}(P), Q$ by $\varphi(Q)=\varphi^{\prime}(Q)$, and $\varphi$ by $\varphi \circ\left(\varphi^{\prime}\right)^{-1}$, we can assume that $\varphi^{\prime}=\operatorname{incl}_{P}^{S}$ and $\left.\varphi\right|_{Q}=\operatorname{Id}_{Q}$. We must show that $\varphi=c_{x}$ for some $x \in Z(Q)$.

Set $K=\operatorname{Aut}_{P}(Q)$. Since $Q$ is $\mathcal{F}$-centric, it is fully centralized. Since $\operatorname{Aut}_{\mathcal{F}}^{K}(Q)=$ Aut $_{S}^{K}(Q)=K, Q$ is fully $K$-automized, and hence it is fully $K$-normalized by Lemma 2.2(a,b). Hence by Theorem 2.3, the normalizer subsystem $N_{\mathcal{F}}^{K}(Q)$ over $N_{S}^{K}(Q)=P \cdot C_{S}(Q)=P$ is saturated. Also, since $\left.\varphi\right|_{Q}=\operatorname{Id}, \operatorname{Aut}_{\varphi(P)}(Q)=$ $\operatorname{Aut}_{P}(Q)=K$. Thus $\varphi(P) \leq N_{S}^{K}(Q)$, and $\varphi \in \operatorname{Mor}\left(N_{\mathcal{F}}^{K}(Q)\right)$. Set $\mathcal{F}_{0}=N_{\mathcal{F}}^{K}(Q)$ for short. 
It thus suffices to prove that $\mathcal{F}_{0}=\mathcal{F}_{P}(P)$. Assume otherwise: then by [9, Theorem 3.6], there is $R \leq P$ such that $O_{p}\left(\operatorname{Aut}_{\mathcal{F}_{0}}(R)\right)=1$, and in particular, such that $\operatorname{Aut}_{\mathcal{F}_{0}}(R)$ is not a $p$-group. By definition of $\mathcal{F}_{0}$, we can assume that $R \geq Q$. Hence there is $\mathrm{Id} \neq \alpha \in \operatorname{Aut}_{\mathcal{F}_{0}}(R)$ which has finite order prime to $p$. Since $K$ is a discrete $p$-toral group and $\left.\alpha\right|_{Q} \in K,\left.\alpha\right|_{Q}=\operatorname{Id}_{Q}$. Hence for $g \in R, g$ and $\alpha(g)$ have the same conjugation action on $Q$, and $g^{-1} \alpha(g) \in C_{R}(Q) \leq Q$. Thus $\alpha$ induces the identity on $R / Q$, so by [9, Lemma 1.7(a)], each $\alpha$-orbit in $R$ has $p$-power order. This contradicts the assumption that $\alpha \neq$ Id has order prime to $p$, so $\mathcal{F}_{0}=\mathcal{F}_{P}(P)$, and this finishes the proof.

We will need the following application of Lemma 2.4.

Lemma 2.5 Let $\mathcal{F}$ be a saturated fusion system over a discrete $p$-toral group $S$. Then for each $P \leq S$, the set of $S$-conjugacy classes of fully normalized subgroups $\mathcal{F}$-conjugate to $P$ is finite of order prime to $p$.

Proof Since the conclusion depends only on the $\mathcal{F}$-conjugacy class of $P$, we can assume that $P$ is fully normalized.

Let $\operatorname{Rep}_{\mathcal{F}}(P, S)_{\mathrm{fc}} \subseteq \operatorname{Rep}_{\mathcal{F}}(P, S)$ be the subset consisting of all classes of homomorphisms whose image is fully centralized. $\operatorname{Recall} \operatorname{Rep}_{\mathcal{F}}(P, S)$ is finite [9, Lemma 2.5].

Step 1 Assume first $P$ is $\mathcal{F}$-centric. We first $\operatorname{prove}^{\operatorname{Rep}_{\mathcal{F}}}(P, S)_{\mathrm{fc}}=\operatorname{Rep}_{\mathcal{F}}(P, S)$ has order prime to $p$ in this case.

Recall from [9, Section 3] that there is a functor $\left(P \mapsto P^{\bullet}\right)$ from $\mathcal{F}$ to itself, with the properties that $P^{\bullet} \geq P$ for each $P \leq S,\left(P^{\bullet}\right)^{\bullet}=P^{\bullet}$, and the image $\mathcal{F}^{\bullet}$ of the functor contains finitely many $S$-conjugacy classes. (We will be studying this functor in more detail in the next section.)

Let $P=P_{0}<P_{1}<P_{2}<\cdots<P_{m}=S$ be such that $P_{i}=N_{S}\left(P_{i-1}\right)^{\bullet}$ for each $i$. The sequence is finite since there are only finitely many conjugacy classes in $\mathcal{F}^{\bullet}$ (and since $N_{S}\left(P_{i}\right)>P_{i}$ whenever $P_{i}<S$ by Lemma 1.12). Fix $0 \leq i<m$, and consider the restriction map

$$
\text { Res: } \operatorname{Rep}_{\mathcal{F}}\left(N_{S}\left(P_{i}\right), S\right) \longrightarrow \operatorname{Rep}_{\mathcal{F}}\left(P_{i}, S\right) .
$$

This is injective by Lemma 2.4, and $\operatorname{Out}_{S}\left(P_{i}\right) \cong N_{S}\left(P_{i}\right) / P_{i} \neq 1$ is a finite $p$-group by [9, Proposition 1.5(c)]. Let $Q / P_{i}$ be the stabilizer subgroup of $[\varphi] \in \operatorname{Rep}_{\mathcal{F}}\left(P_{i}, S\right)$ under the action of $N_{S}\left(P_{i}\right) / P_{i}$; then $\varphi$ extends to an $\mathcal{F}$-morphism on $Q$ by axiom (II) in Definition 1.4. Thus $\operatorname{Im}\left(\right.$ Res) is the fixed subset for the $N_{S}\left(P_{i}\right) / P_{i}$-action, and hence $\left|\operatorname{Rep}_{\mathcal{F}}\left(P_{i}, S\right)\right| \equiv\left|\operatorname{Rep}_{\mathcal{F}}\left(N_{S}\left(P_{i}\right), S\right)\right|(\bmod p)$. 
By [9, Proposition 3.3], each morphism $\psi \in \operatorname{Hom}_{\mathcal{F}}\left(N_{S}\left(P_{i}\right), S\right)$ extends to a unique morphism $\psi^{\bullet} \in \operatorname{Hom}_{\mathcal{F}}\left(N_{S}\left(P_{i}\right)^{\bullet}, S\right)$. Therefore, restriction defines a bijection from $\operatorname{Hom}_{\mathcal{F}}\left(N_{S}\left(P_{i}\right)^{\bullet}, S\right)$ to $\operatorname{Hom}_{\mathcal{F}}\left(N_{S}\left(P_{i}\right), S\right)$, and hence (after dividing out by the conjugation action of $S)$ from $\operatorname{Rep}_{\mathcal{F}}\left(N_{S}\left(P_{i}\right)^{\bullet}, S\right)$ to $\operatorname{Rep}_{\mathcal{F}}\left(N_{S}\left(P_{i}\right), S\right)$. In other words, $\left|\operatorname{Rep}_{\mathcal{F}}\left(P_{i}, S\right)\right| \equiv\left|\operatorname{Rep}_{\mathcal{F}}\left(P_{i+1}, S\right)\right|(\bmod p)$. Thus

$$
\left|\operatorname{Rep}_{\mathcal{F}}(P, S)\right|=\left|\operatorname{Rep}_{\mathcal{F}}\left(P_{0}, S\right)\right| \equiv\left|\operatorname{Rep}_{\mathcal{F}}\left(P_{m}, S\right)\right|=\left|\operatorname{Out}_{\mathcal{F}}(S)\right|,
$$

where $\left|\operatorname{Out}_{\mathcal{F}}(S)\right|$ is prime to $p$ by axiom (I) in Definition 1.4.

Step 2 Now assume $P<S$ is not $\mathcal{F}$-centric; we claim that $\left|\operatorname{Rep}_{\mathcal{F}}(P, S)_{\text {fc }}\right|$ is prime to $p$. Set

$$
\Gamma=\left\{\gamma \in \operatorname{Aut}_{\mathcal{F}}\left(P C_{S}(P)\right)|\gamma|_{P}=\operatorname{Id}_{P}\right\} \quad \text { and } \quad T=\operatorname{Aut}_{C_{S}(P)}\left(P C_{S}(P)\right) \unlhd \Gamma .
$$

Then $\Gamma \cong \operatorname{Aut}_{C_{\mathcal{F}}(P)}\left(C_{S}(P)\right)$ and $T \cong \operatorname{Inn}\left(C_{S}(P)\right)$ via restriction to $C_{S}(P)$, so $T \in \operatorname{Syl}_{p}(\Gamma)$.

Now $\Gamma / T \leq \operatorname{Out}_{\mathcal{F}}\left(P C_{S}(P)\right)$ acts on $\operatorname{Rep}_{\mathcal{F}}\left(P C_{S}(P), S\right)$ by right composition. If $\varphi \in$ $\operatorname{Hom}_{\mathcal{F}}\left(P C_{S}(P), S\right)$ and $\gamma \in \Gamma$ are such that $[\varphi]=[\varphi \circ \gamma]$ in $\operatorname{Rep}_{\mathcal{F}}\left(P C_{S}(P), S\right)$, then there is $g \in S$ such that $\varphi=c_{g} \circ \varphi \circ \gamma$, so $\varphi=\varphi \circ \gamma^{p^{n}}$ for some $n$, hence $|\gamma|$ is a power of $p$, and $\gamma \in T$. Thus the $\Gamma / T$-action on $\operatorname{Rep}_{\mathcal{F}}\left(P C_{S}(P), S\right)$ is free. The restriction map from $\operatorname{Rep}_{\mathcal{F}}\left(P C_{S}(P), S\right)$ to $\operatorname{Rep}_{\mathcal{F}}(P, S)_{\text {fc }}$ is surjective by axiom (II), and the inverse image of any $[\psi] \in \operatorname{Rep}_{\mathcal{F}}(P, S)_{\mathrm{fc}}$ is one of the $\Gamma / T$-orbits. Thus $\left|\operatorname{Rep}_{\mathcal{F}}(P, S)_{\mathrm{fc}}\right|=\left|\operatorname{Rep}_{\mathcal{F}}\left(P C_{S}(P), S\right)\right| /|\Gamma / T|$. Since $\left|\operatorname{Rep}_{\mathcal{F}}\left(P C_{S}(P), S\right)\right|$ is prime to $p$ by Step $1,\left|\operatorname{Rep}_{\mathcal{F}}(P, S)_{\mathrm{fc}}\right|$ is also prime to $p$.

Step 3 We are now ready to prove the lemma. Let $\mathcal{P}_{\mathrm{fc}}$ be the set of $S$-conjugacy classes of subgroups fully centralized in $\mathcal{F}$ and $\mathcal{F}$-conjugate to $P$, and let $\mathcal{P}_{\text {fn }} \subseteq \mathcal{P}_{\text {fc }}$ be the subset of classes of fully normalized subgroups. Let $\rho: \operatorname{Rep}_{\mathcal{F}}(P, S)_{\mathrm{fc}} \rightarrow \mathcal{P}_{\mathrm{fc}}$ be the map which sends the class of $\varphi$ to the class of $\varphi(P)$.

Let $N|| \operatorname{Out}_{\mathcal{F}}(P) \mid$ be the largest divisor prime to $p$. For each $Q \in P^{\mathcal{F}}$ such that $[Q] \in \mathcal{P}_{\mathrm{fc}},\left|\rho^{-1}([Q])\right|=\left|\operatorname{Aut}_{\mathcal{F}}(Q)\right| /\left|\operatorname{Aut}_{S}(Q)\right|$. Thus $p|| \rho^{-1}(Q) \mid$ if $Q$ is not fully normalized, and $\left|\rho^{-1}(Q)\right|=N$ if $Q$ is fully normalized. So $\left|\rho^{-1}\left(\mathcal{P}_{\mathrm{fn}}\right)\right| \equiv$ $\left|\operatorname{Rep}_{\mathcal{F}}(P, S)_{\mathrm{fc}}\right|(\bmod p)$, hence is prime to $p$ by Steps 1 and 2 ; and thus $\left|\mathcal{P}_{\mathrm{fn}}\right|=$ $\left|\rho^{-1}\left(\mathcal{P}_{\mathrm{fn}}\right)\right| / N$ is also prime to $p$.

\section{The "bullet" construction}

The bullet construction plays an important role in the theory of $p$-local compact groups, and is studied at length in [9, Section 3]. However, in that paper, we always assumed 
that the ambient fusion system is saturated. In this one, we will need some of the properties of this construction to prove saturation in certain situations, and hence must know them in a more general setting. We start by recalling the definition.

Definition 3.1 Fix a discrete $p$-toral group $S$, set $T=S_{0}$, and let $W \leq \operatorname{Aut}(T)$ be a subgroup which contains $\operatorname{Aut}_{S}(T)$. Let $m \geq 0$ be such that $\exp (S / T)=p^{m}$.

- For each $A \leq T$, set

$$
I(A)=C_{T}\left(C_{W}(A)\right):=\left\{t \in T \mid w(t)=t \text { for all } w \in C_{W}(A)\right\} \geq A .
$$

- For each $P \leq S$, set $P^{[m]}=\left\langle g p^{m} \mid g \in P\right\rangle$, and set $P^{\bullet}=P \cdot I\left(P^{[m]}\right)_{0}$.

- Let $P, Q \leq S$ and $\varphi \in \operatorname{Inj}(P, Q)$ be such that $\left.\varphi\right|_{P^{[m]}}=\left.w\right|_{\left.P^{[m]}\right]}$ for some $w \in W$. Then $\varphi^{\bullet}: P^{\bullet} \rightarrow Q^{\bullet}$ denotes the unique map of sets, if it exists, such that $\varphi^{\bullet}(g h)=\varphi(g) w(h)$ for each $g \in P$ and $h \in I\left(P^{[m]}\right)_{0}$.

Note that the definition of $P^{\bullet}$ for $P \leq S$ depends only on $S$ and on the choice of a subgroup $W \leq \operatorname{Aut}\left(S_{0}\right)$. For any fusion system $\mathcal{F}$ over $S$, we associate to $\mathcal{F}$ the construction $\left(P \mapsto P^{\bullet}\right)$ with $W=\operatorname{Aut}_{\mathcal{F}}\left(S_{0}\right)$, and set $\mathcal{F}^{\bullet}=\left\{P^{\bullet} \mid P \leq S\right\}$.

We will show later that under certain additional conditions, the map $\varphi^{\bullet}$ is defined and a homomorphism for each morphism $\varphi$.

Lemma 3.2 Let $\mathcal{F}$ be a fusion system over a discrete $p$-toral group $S$. Then we have the following.

(a) $P \leq Q \leq S$ implies $P^{\bullet} \leq Q^{\bullet}$.

(b) $P \leq S$ implies $\left(P^{\bullet}\right)^{\bullet}=P^{\bullet}$ and $N_{S}(P) \leq N_{S}\left(P^{\bullet}\right)$.

The following also hold if $\operatorname{Aut}_{\mathcal{F}}\left(S_{0}\right)$ is finite.

(c) The set $\mathcal{F}^{\bullet}$ contains finitely many $S$-conjugacy classes. $N>0$ such that $P_{i}^{\bullet}=P^{\bullet}$ for each $i>N$.

Proof By definition, the function $P \mapsto P^{\bullet}$ depends only on $\operatorname{Aut}_{\mathcal{F}}\left(S_{0}\right)$ (and on $S$ ). Hence (a), (b) and (c) hold by exactly the same arguments as those used to prove them in [9, Lemma 3.2]. The only exception is the last statement in (b) (not shown in [9]), which follows since for $g \in N_{S}(P), g \in N_{S}\left(P^{[m]}\right)$, and hence $g \in N_{S}\left(I\left(P^{[m]}\right)\right)$. Note that the assumption $\left|\operatorname{Aut}_{\mathcal{F}}\left(S_{0}\right)\right|<\infty$ is needed to prove (c). 
In the situation of (d), we have $P_{1}^{\bullet} \leq P_{2}^{\bullet} \leq \cdots$ by (a), and this sequence contains only finitely many subgroups by (c). Thus there are $N>0$ and $Q \leq S$ such that $P_{i}^{\bullet}=Q$ for each $i>N$. In particular, $P=\bigcup_{i=1} P_{i} \leq Q$, so $P^{\bullet} \leq Q^{\bullet}=Q$ by (a) and (b). Since $P_{i}^{\bullet} \leq P^{\bullet}$ for each $i$ by (a) again, this shows that $P^{\bullet}=Q=P_{i}^{\bullet}$ for $i>N$.

While we do not assume here that fusion systems are saturated, we will, in most cases, assume the following condition on a fusion system $\mathcal{F}$ :

(*) For each $P \leq S_{0}$ and each $\varphi \in \operatorname{Hom}_{\mathcal{F}}\left(P, S_{0}\right), \varphi=\left.w\right|_{P}$ for some $w \in \operatorname{Aut}_{\mathcal{F}}\left(S_{0}\right)$.

By [9, Lemma 2.4(b)], (*) always holds if $\mathcal{F}$ is saturated.

Lemma 3.3 Let $\mathcal{F}$ be a fusion system over a discrete $p$-toral group $S$ such that $\mathcal{F}$ satisfies (*). Then for each $P \leq S$ and each $\varphi \in \operatorname{Hom}_{\mathcal{F}}\left(P^{\bullet}, S\right), \varphi\left(P^{\bullet}\right)=\varphi(P)^{\bullet} \in \mathcal{F}^{\bullet}$. In particular, $\mathcal{F}^{\bullet}$ is invariant under $\mathcal{F}$-conjugacy.

Proof Let $m$ be such that $p^{m}=\exp \left(S / S_{0}\right)$, and set $W=\operatorname{Aut}_{\mathcal{F}}\left(S_{0}\right)$. Set $Q=\varphi(P)$. We must show that $\varphi\left(P^{\bullet}\right)=Q^{\bullet}$.

Set $R=P^{\bullet} \cap S_{0} \cap \varphi^{-1}\left(S_{0}\right)$. Thus $R$ is the largest subgroup of $P^{\bullet}$ such that $R$ and $\varphi(R)$ are both contained in $S_{0}$. By $(*)$, there is $w \in \operatorname{Aut}_{\mathcal{F}}\left(S_{0}\right)$ such that $\left.\varphi\right|_{R}=\left.w\right|_{R}$. In particular, $w\left(P^{[m]}\right)=Q^{[m]}$, so $w C_{W}\left(P^{[m]}\right) w^{-1}=C_{W}\left(Q^{[m]}\right)$, and $w$ sends $I\left(P^{[m]}\right)=C_{T}\left(C_{W}\left(P^{[m]}\right)\right)$ isomorphically to $I\left(Q^{[m]}\right)$. Also, $\varphi\left(I\left(P^{[m]}\right)_{0}\right) \leq S_{0}$ since it is connected, so $I\left(P^{[m]}\right)_{0} \leq R$ and $\varphi\left(I\left(P^{[m]}\right)_{0}\right)=w\left(I\left(P^{[m]}\right)_{0}\right)=I\left(Q^{[m]}\right)_{0}$. So $\varphi\left(P^{\bullet}\right)=Q^{\bullet}$.

Throughout the rest of the section, we need to consider the following condition on a fusion system $\mathcal{F}$ over a discrete $p$-toral group $S$ :

$(* *)$

For each $P \leq S$ and each $\varphi \in \operatorname{Hom}_{\mathcal{F}}\left(P, S_{0}\right)$, there exists $\bar{\varphi} \in \operatorname{Hom}_{\mathcal{F}}\left(P \cdot C_{S}(P)_{0}, S_{0}\right)$ such that $\left.\bar{\varphi}\right|_{P}=\varphi$.

As will be seen in the next lemma, this is a stronger version of $(*)$.

Lemma 3.4 Fix a fusion system $\mathcal{F}$ over a discrete $p$-toral group $S$ which satisfies $(* *)$. Then the following hold.

(a) The fusion system $\mathcal{F}$ satisfies condition $(*)$.

(b) For each $P, R \leq S_{0}$ such that $P \leq R \leq P \cdot I(P)_{0}$, each $w \in W$, and each $\psi \in \operatorname{Hom}_{\mathcal{F}}(R, S)$ such that $\left.\psi\right|_{P}=\left.w\right|_{P}, \psi(R) \leq S_{0}$ and $\psi=\left.w\right|_{R}$.

(c) For each $P, Q \leq S$ and each $\varphi \in \operatorname{Hom}_{\mathcal{F}}(P, Q), \varphi^{\bullet}$ is defined and $\varphi^{\bullet} \in$ $\operatorname{Hom}\left(P^{\bullet}, Q^{\bullet}\right)$.

(d) Assume $P \leq Q \leq P^{\bullet}, \varphi \in \operatorname{Hom}_{\mathcal{F}}(P, S)$, and $\bar{\varphi} \in \operatorname{Hom}_{\mathcal{F}}(Q, S)$ are such that $\varphi=\left.\bar{\varphi}\right|_{P}$. Then $\bar{\varphi}=\left.\varphi^{\bullet}\right|_{Q}$. 
Proof Set $W=\operatorname{Aut}_{\mathcal{F}}\left(S_{0}\right)$. Let $m$ be such that $p^{m}=\exp \left(S / S_{0}\right)$.

(a) For each $P \leq S_{0}$ and $\varphi \in \operatorname{Hom}_{\mathcal{F}}\left(P, S_{0}\right), \varphi$ extends to $w \in \operatorname{Aut}_{\mathcal{F}}\left(S_{0}\right)$ by (**).

(b) Let $P \leq S_{0}, P \leq R \leq P \cdot I(P)_{0}, w \in W$, and $\psi \in \operatorname{Hom}_{\mathcal{F}}(R, S)$ be as above. Set $P_{*}=\psi(P)=w(P)$ and $R_{*}=\psi(R)$. Then $P_{*} \leq R_{*}$ are abelian, so each element of $\operatorname{Aut}_{R_{*}}\left(S_{0}\right) \leq W$ acts via the identity on $P_{*}$, and hence via the identity on $I\left(P_{*}\right)$. In other words, $\left[I\left(P_{*}\right), R_{*}\right]=1$. By $(* *), \psi^{-1} \in \operatorname{Iso}_{\mathcal{F}}\left(R_{*}, R\right)$ extends to a morphism $\chi \in \operatorname{Hom}_{\mathcal{F}}\left(R_{*} \cdot I\left(P_{*}\right)_{0}, S_{0}\right)$.

Now, $\chi\left(P_{*}\right)=P$, and $\left.\chi\right|_{P_{*}}=\left.\psi^{-1}\right|_{P_{*}}=\left.w^{-1}\right|_{P_{*}}$. Also, $\left.\chi\right|_{P_{*} \cdot I\left(P_{*}\right)_{0}}=\left.u\right|_{P_{*} \cdot I\left(P_{*}\right)_{0}}$ for some $u \in W$ by (a), $\left.u\right|_{P_{*}}=\left.w^{-1}\right|_{P_{*}}$, and hence $\left.u\right|_{I\left(P_{*}\right)}=\left.w^{-1}\right|_{I\left(P_{*}\right)}$ by definition of $I(-)$. Thus $\left.\chi\right|_{P_{*} \cdot I\left(P_{*}\right)_{0}}=\left.w^{-1}\right|_{P_{*} \cdot I\left(P_{*}\right)_{0}}$, and so $\chi\left(P_{*} \cdot I\left(P_{*}\right)_{0}\right)=P \cdot I(P)_{0} \geq R$. Since $\chi$ is injective and $\chi\left(R_{*}\right)=R$, this implies that $R_{*} \leq P_{*} \cdot I\left(P_{*}\right)_{0} \leq S_{0}$ and $\psi=\left(\left.\chi\right|_{R_{*}}\right)^{-1}=\left.w\right|_{R}$.

(c) Fix a morphism $\varphi \in \operatorname{Hom}_{\mathcal{F}}(P, Q)$, and let $w \in W$ be such that $\left.\varphi\right|_{P^{[m]}}=\left.w\right|_{P^{[m]}}$. Set $R=P^{[m]} \cdot\left(P \cap I\left(P^{[m]}\right)_{0}\right)$. Then $P^{[m]} \leq R \leq P^{[m]} \cdot I\left(P^{[m]}\right)_{0}$, and (b) implies $\left.\varphi\right|_{R}=\left.w\right|_{R}$. Hence $\varphi^{\bullet}: P^{\bullet} \rightarrow Q^{\bullet}$ is well defined as a map of sets by the formula $\varphi^{\bullet}(g h)=\varphi(g) w(h)$ for all $g \in P$ and $h \in I\left(P^{[m]}\right)_{0}$.

To prove that $\varphi^{\bullet}$ is a homomorphism, it remains to show, for all $g \in P$ and $h \in I\left(P^{[m]}\right)_{0}$, that $w\left(g h g^{-1}\right)=\varphi(g) w(h) \varphi(g)^{-1}$. Set $u=c_{\varphi(g)}^{-1} \circ w \circ c_{g} \in W$; we must show

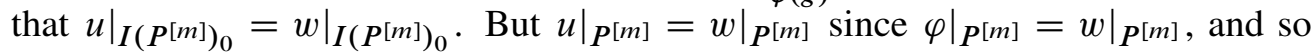
$\left.u\right|_{I\left(P^{[m]}\right)}=\left.w\right|_{I\left(P^{[m]}\right)}$ by definition of $I(-)$.

(d) Fix $P, Q \leq S$ such that $P \leq Q \leq P^{\bullet}$. Assume $\varphi \in \operatorname{Hom}_{\mathcal{F}}(P, S)$ and $\bar{\varphi} \in$ $\operatorname{Hom}_{\mathcal{F}}(Q, S)$ are such that $\varphi=\left.\bar{\varphi}\right|_{P}$. Set $R=P^{[m]}$ and $Q^{\prime}=Q \cap R \cdot I(R)_{0}$. By definition, $\left.\varphi^{\bullet}\right|_{R \cdot I(R)_{0}}=\left.w\right|_{R \cdot I(R)_{0}}$ for any $w \in W$ such that $\left.\varphi\right|_{R}=\left.w\right|_{R}$. By (b), $\left.\bar{\varphi}\right|_{Q^{\prime}}=\left.w\right|_{Q^{\prime}}=\left.\varphi^{\bullet}\right|_{Q^{\prime}}$. Since

$$
Q=Q \cap P^{\bullet}=Q \cap\left(P \cdot I(R)_{0}\right)=P \cdot\left(Q \cap R \cdot I(R)_{0}\right)=P Q^{\prime}
$$

and $\left.\bar{\varphi}\right|_{P}=\varphi$, this proves that $\bar{\varphi}=\left.\varphi^{\bullet}\right|_{Q}$.

Lemma 3.4(c) allows us to extend each $\varphi \in \operatorname{Hom}_{\mathcal{F}}(P, Q)$ to a homomorphism $\varphi^{\bullet} \in$ $\operatorname{Hom}\left(P^{\bullet}, Q^{\bullet}\right)$. In the next lemma, we add some more hypotheses, enough to ensure that $\varphi^{\bullet} \in \operatorname{Mor}(\mathcal{F})$.

Lemma 3.5 Fix a fusion system $\mathcal{F}$ over a discrete $p$-toral group $S$. Let $\mathcal{H}$ be a family of subgroups of $S$ invariant under $\mathcal{F}$-conjugacy. Assume the following.

(i) $\operatorname{Aut}_{\mathcal{F}}\left(S_{0}\right)$ is finite.

(ii) $\mathcal{F}$ satisfies $(* *)$. 
(iii) For all $P \in \mathcal{H}$ and $P \leq Q \leq P^{\bullet}, Q \in \mathcal{H}$.

(iv) $\mathcal{F}$ is $\mathcal{H}$-generated and $\mathcal{H}$-saturated.

Then the following hold.

(a) For each $\varphi \in \operatorname{Mor}(\mathcal{F}), \varphi^{\bullet} \in \operatorname{Mor}(\mathcal{F})$.

(b) If, in addition, $\widehat{\mathcal{H}} \supseteq \mathcal{H}$ is also invariant under $\mathcal{F}$-conjugacy, and is such that $P \in \widehat{\mathcal{H}}$ implies $P^{\bullet} \in \mathcal{H}$, then $\mathcal{F}$ is $\widehat{\mathcal{H}}$-saturated.

Proof (a) Fix $\varphi \in \operatorname{Hom}_{\mathcal{F}}(P, Q)$; we must show $\varphi^{\bullet} \in \operatorname{Hom}_{\mathcal{F}}\left(P^{\bullet}, Q^{\bullet}\right)$. It suffices to do this when $\varphi$ is an isomorphism. Since $\mathcal{F}$ is $\mathcal{H}$-generated, $\varphi$ is a composite of isomorphisms which are restrictions of $\mathcal{F}$-isomorphisms between subgroups in $\mathcal{H}$. So it suffices to consider the case where $\varphi$ extends to $\bar{\varphi} \in \operatorname{Iso}_{\mathcal{F}}(\bar{P}, \bar{Q})$ for some $\bar{P}, \bar{Q} \in \mathcal{H}$. If $\bar{\varphi}^{\bullet} \in \operatorname{Mor}(\mathcal{F})$, then $\varphi^{\bullet} \in \operatorname{Mor}(\mathcal{F})$ since it is a restriction of $\bar{\varphi}^{\bullet}$. It thus suffices to prove (a) when $P$ and $Q$ are in $\mathcal{H}$.

By axiom (III), which holds by hypothesis for all subgroups in $\mathcal{H}$, there is a subgroup $P^{\prime} \leq P^{\bullet}$ containing $P$ which is maximal among subgroups of $P^{\bullet}$ satisfying $\varphi^{\prime}:=$ $\left.\varphi^{\bullet}\right|_{P^{\prime}} \in \operatorname{Hom}_{\mathcal{F}}\left(P^{\prime}, Q^{\bullet}\right)$. Assume $P^{\prime}<P^{\bullet}$, and set $Q^{\prime}=\varphi^{\prime}\left(P^{\prime}\right)$.

Since $P, Q \in \mathcal{H}$, and since $P \leq P^{\prime} \leq P^{\bullet}$ and $Q \leq Q^{\prime} \leq Q^{\bullet}, P^{\prime}, Q^{\prime} \in \mathcal{H}$ by (iii). Since $\mathcal{F}$ is $\mathcal{H}$-saturated, there are $R^{\prime} \leq S$ and $\psi^{\prime} \in \operatorname{Iso}_{\mathcal{F}}\left(Q^{\prime}, R^{\prime}\right)$ such that $R^{\prime}$ is receptive in $\mathcal{F}$. Set $R=\psi^{\prime}(Q)$ and $\psi=\left.\psi^{\prime}\right|_{Q} \in \operatorname{Iso}_{\mathcal{F}}(Q, R)$. By Lemma 3.4(d), $\psi^{\prime}=\left.\psi^{\bullet}\right|_{Q^{\prime}}$, and $\psi^{\prime} \varphi^{\prime}=\left.(\psi \varphi)^{\bullet}\right|_{P^{\prime}}$. We thus have isomorphisms of triples

$$
\left(P^{\bullet} \geq P^{\prime} \geq P\right) \underset{\left(\varphi^{\bullet}, \varphi^{\prime}, \varphi\right)}{\cong}\left(Q^{\bullet} \geq Q^{\prime} \geq Q\right) \stackrel{\left(\psi^{\bullet}, \psi^{\prime}, \psi\right)}{\cong}\left(R^{\bullet} \geq R^{\prime} \geq R\right) .
$$

Set $P^{\prime \prime}=N_{P} \cdot\left(P^{\prime}\right)$ and $Q^{\prime \prime}=N_{Q} \cdot\left(Q^{\prime}\right)$. Since $P^{\bullet}>P^{\prime}, P^{\prime \prime}>P^{\prime}$ and $Q^{\prime \prime}>Q^{\prime}$ by Lemma 1.12. Also, $N_{\psi^{\prime}} \geq Q^{\prime \prime}$ and $N_{\psi^{\prime} \varphi^{\prime}} \geq P^{\prime \prime}$ since there are (abstract) homomorphisms which extend $\psi^{\prime}$ to $Q^{\prime \prime}$ and $\psi^{\prime} \varphi^{\prime}$ to $P^{\prime \prime}$. Hence $\psi^{\prime}$ and $\psi^{\prime} \varphi^{\prime}$ extend to homomorphisms $\psi^{\prime \prime} \in \operatorname{Hom}_{\mathcal{F}}\left(Q^{\prime \prime}, S\right)$ and $\chi^{\prime \prime} \in \operatorname{Hom}_{\mathcal{F}}\left(P^{\prime \prime}, S\right)$, and $\psi^{\prime \prime}=\left.\psi^{\bullet}\right|_{Q^{\prime \prime}}$ and $\chi^{\prime \prime}=\left.(\psi \varphi)^{\bullet}\right|_{P^{\prime \prime}}$ by Lemma 3.4(d) again. So $\psi^{\prime \prime}\left(Q^{\prime \prime}\right)=\chi^{\prime \prime}\left(P^{\prime \prime}\right)=N_{R^{\bullet}}\left(R^{\prime}\right)$, and $\left.\varphi^{\bullet}\right|_{P^{\prime \prime}}=\left(\psi^{\prime \prime}\right)^{-1} \chi^{\prime \prime}$ is a morphism in $\mathcal{F}$, which contradicts the maximality of $P^{\prime}$. We now conclude that $P^{\prime}=P^{\bullet}$, and hence that $\varphi^{\bullet} \in \operatorname{Iso}_{\mathcal{F}}\left(P^{\bullet}, Q^{\bullet}\right)$.

(b) We are assuming $\mathcal{F}$ is $\mathcal{H}$-saturated, and want to show it is $\hat{\mathcal{H}}$-saturated. Fix $P$ in $\hat{\mathcal{H}} \backslash \mathcal{H}$. Thus $P^{\bullet} \in \mathcal{H}$ by assumption. Choose $R$ which is $\mathcal{F}$-conjugate to $P^{\bullet}$, and receptive and fully automized. Fix $\varphi \in \operatorname{Iso}_{\mathcal{F}}\left(P^{\bullet}, R\right)$ and set $Q=\varphi(P)$. By Lemma 3.4(d), $\varphi=\left(\left.\varphi\right|_{P}\right)^{\bullet}$, and so $Q^{\bullet}=R$.

Set $H=\left\{\alpha \in \operatorname{Aut}_{\mathcal{F}}\left(Q^{\bullet}\right) \mid \alpha(Q)=Q\right\}$. Since $Q^{\bullet}=R$ is fully automized, $\operatorname{Aut}_{S}\left(Q^{\bullet}\right) \in$ $\operatorname{Syl}_{p}\left(\operatorname{Aut}_{\mathcal{F}}\left(Q^{\bullet}\right)\right)$. Thus $H$ acts by translation on $\operatorname{Aut}_{\mathcal{F}}\left(Q^{\bullet}\right) / \operatorname{Aut}_{S}\left(Q^{\bullet}\right)$, a finite set 
of order prime to $p$, and there is $\beta \in \operatorname{Aut}_{\mathcal{F}}\left(Q^{\bullet}\right)$ such that the coset $\beta^{-1} \operatorname{Aut}_{S}\left(Q^{\bullet}\right)$ is in an $H$-orbit of order prime to $p$. In other words, the stabilizer subgroup $\beta^{-1} \operatorname{Aut}_{S}\left(Q^{\bullet}\right) \beta \cap H$ has index prime to $p$ in $H$, and thus lies in $\operatorname{Syl}_{p}(H)$.

By (a) (and the definition of $H$ ), restriction to $Q$ sends $\beta H \beta^{-1}$ surjectively onto $\operatorname{Aut}_{\mathcal{F}}(\beta(Q))$. Also, $\operatorname{Aut}_{S}(\beta(Q))$ is the image of $\operatorname{Aut}_{S}\left(Q^{\bullet}\right) \cap \beta H \beta^{-1}$ under this surjection, and hence is a Sylow $p$-subgroup in $\operatorname{Aut}_{\mathcal{F}}(\beta(Q))$. Thus $\beta(Q)$ is fully automized. (Note that $\beta(Q)^{\bullet}=Q^{\bullet}$.)

For any $R \in P^{\mathcal{F}}=Q^{\mathcal{F}}$ and any $\varphi \in \operatorname{Iso}_{\mathcal{F}}(R, Q), N_{\varphi} \leq N_{\varphi} \bullet:$ if $\varphi c_{g} \varphi^{-1}=c_{h} \in$ $\operatorname{Aut}_{S}(Q)$, then $\varphi^{\bullet} c_{g} \varphi^{\bullet-1}=c_{h} \in \operatorname{Aut}_{S}\left(Q^{\bullet}\right)$ by Lemma 3.2(d). Hence $Q$ and $\beta(Q)$ are both receptive in $\mathcal{F}$ since $Q^{\bullet}$ is. Thus the conjugacy class $P^{\mathcal{F}}$ contains a subgroup which is receptive and fully automized.

Assume $P_{\infty}=\bigcup_{i=1} P_{i}$, where $\left\{P_{i}\right\}$ is an increasing sequence of subgroups in $\hat{\mathcal{H}}$. Fix $\varphi \in \operatorname{Hom}_{\mathcal{F}}\left(P_{\infty}, S\right)$ such that $\left.\varphi\right|_{P_{i}} \in \operatorname{Hom}_{\mathcal{F}}\left(P_{i}, S\right)$ for each $i$. By Lemma 3.2(d), for $i$ large enough, $P_{i}^{\bullet}=P_{\infty}^{\bullet}$. By Lemma 3.4(d), $\varphi$ is the restriction of $\left(\left.\varphi\right|_{P_{i}}\right)^{\bullet}$, which is in $\mathcal{F}$ by (a). So $\varphi \in \operatorname{Hom}_{\mathcal{F}}\left(P_{\infty}, S\right)$.

\section{Centric and radical subgroups determine saturation}

The main result in this section is Theorem 4.2, which gives sufficient conditions for a fusion system over a discrete $p$-toral group to be saturated.

We will frequently refer to the conditions $(*)$ and $(* *)$ of Section 3 , which we recall here:

(*) For all $P \leq S_{0}$ and $\varphi \in \operatorname{Hom}_{\mathcal{F}}\left(P, S_{0}\right), \varphi=\left.w\right|_{P}$ for some $w \in \operatorname{Aut}_{\mathcal{F}}\left(S_{0}\right)$.

$(* *)$ For all $P \leq S$ and $\varphi \in \operatorname{Hom}_{\mathcal{F}}\left(P, S_{0}\right)$,

there exists $\bar{\varphi} \in \operatorname{Hom}_{\mathcal{F}}\left(P \cdot C_{S}(P)_{0}, S_{0}\right)$ with $\left.\bar{\varphi}\right|_{P}=\varphi$.

By Lemma 3.4(a), condition (**) implies $(*)$.

The following finiteness result will be needed.

Lemma 4.1 Let $\mathcal{F}$ be a fusion system over a discrete $p$-toral group $S$ such that $\operatorname{Aut}_{\mathcal{F}}\left(S_{0}\right)$ is finite and $(*)$ holds. Then for each $P \leq S$, there are only finitely many $S$-conjugacy classes of subgroups of $S$ which are $\mathcal{F}$-conjugate to $P$.

Proof By $(*)$, and since $\left|\operatorname{Aut}_{\mathcal{F}}\left(S_{0}\right)\right|<\infty, P_{0}^{\mathcal{F}}$ is finite. By [9, Lemma 1.4(a)], for each $R \in P_{0}^{\mathcal{F}}$, there are only finitely many $N_{S}(R) / R$-conjugacy classes of finite subgroups of $N_{S}(R) / R$ of any given order. (Compare [9, Lemma 2.5].) 
The main result in this section is the following theorem. We refer to Definition 1.11 for the definitions of $\mathcal{H}$-saturated and $\mathcal{H}$-generated fusion systems.

Theorem 4.2 Fix a fusion system $\mathcal{F}$ over a discrete $p$-toral group $S$ such that $\operatorname{Aut}_{\mathcal{F}}\left(S_{0}\right)$ is finite and condition $(* *)$ holds. Let $\mathcal{H}$ be a family of subgroups of $S$ which satisfies the following:

(i) $\mathcal{H}$ is invariant under $\mathcal{F}$-conjugacy.

(ii) $\mathcal{H}$ is closed in $\operatorname{Sub}(S)$, and $\mathcal{F}$ is $\mathcal{H}$-generated and $\mathcal{H}$-saturated.

(iii) For all $P \in \mathcal{H}$ and $P \leq Q \leq P^{\bullet}, Q \in \mathcal{H}$.

(iv) If $P \in \mathcal{F}^{\bullet}$ is $\mathcal{F}$-centric and $P \notin \mathcal{H}$, then there is $Q \in P^{\mathcal{F}}$ such that

$$
O_{p}\left(\operatorname{Out}_{\mathcal{F}}(Q)\right) \cap \operatorname{Out}_{S}(Q) \neq 1 \text {. }
$$

Then $\mathcal{F}$ is saturated.

Theorem 4.2 will be shown by following as closely as possible the proof of Castellana, Grodal and the authors [5, Theorem 2.2]. The main difference is that since a discrete $p$-toral group can have infinitely many subgroups, the induction arguments used in [5] cannot be used here. But the beginning steps are mostly unchanged: they are based on the concept of proper $\mathcal{P}$-pairs for an $\mathcal{F}$-conjugacy class $\mathcal{P}$ of subgroups of $S$.

Definition 4.3 Let $\mathcal{F}$ be a fusion system over a discrete $p$-toral group $S$, and let $\mathcal{P}$ be an $\mathcal{F}$-conjugacy class of subgroups of $S$.

- A proper $\mathcal{P}$-pair is a pair of subgroups $(Q, P)$ such that $P<Q \leq N_{S}(P)$ and $P \in \mathcal{P}$.

- Two proper $\mathcal{P}$-pairs $(Q, P)$ and $\left(Q^{\prime}, P^{\prime}\right)$ are $\mathcal{F}$-conjugate if there is an isomorphism $\varphi \in \operatorname{Iso}_{\mathcal{F}}\left(Q, Q^{\prime}\right)$ such that $\varphi(P)=P^{\prime}$. We let $(Q, P)^{\mathcal{F}}$ denote the set of proper $\mathcal{P}$-pairs which are $\mathcal{F}$-conjugate to $(Q, P)$.

- A proper $\mathcal{P}$-pair $(Q, P)$ is fully normalized if $\left|N_{N_{S}(P)}(Q)\right| \geq\left|N_{N_{S}\left(P^{\prime}\right)}\left(Q^{\prime}\right)\right|$ for all $\left(Q^{\prime}, P^{\prime}\right) \in(Q, P)^{\mathcal{F}}$.

Some basic properties of proper $\mathcal{P}$-pairs are shown in the following lemma.

Lemma 4.4 Fix a fusion system $\mathcal{F}$ over a discrete $p$-toral group $S$. Assume that $\mathcal{H} \subseteq \operatorname{Sub}(S)$ is closed, and invariant under $\mathcal{F}$-conjugacy. Assume also that $\mathcal{F}$ is $\mathcal{H}$-generated and $\mathcal{H}$-saturated. Let $\mathcal{P}$ be an $\mathcal{F}$-conjugacy class of subgroups of $S$, maximal among those not in $\mathcal{H}$. 
(a) If $(Q, P)$ is a fully normalized proper $\mathcal{P}$-pair, then $Q$ is receptive in $\mathcal{F}$ and

$$
\operatorname{Aut}_{N_{S}(P)}(Q) \in \operatorname{Syl}_{p}\left(\operatorname{Aut}_{N_{\mathcal{F}}(P)}(Q)\right) .
$$

(b) For each proper $\mathcal{P}$-pair $(Q, P)$, and each $\left(Q^{\prime}, P^{\prime}\right) \in(Q, P)^{\mathcal{F}}$ which is fully normalized, there is a morphism

$$
\varphi \in \operatorname{Hom}_{\mathcal{F}}\left(N_{N_{S}(P)}(Q), N_{S}\left(P^{\prime}\right)\right)
$$

such that $\varphi(P)=P^{\prime}$ and $\varphi(Q)=Q^{\prime}$.

(c) Assume that $\operatorname{Aut}_{\mathcal{F}}\left(S_{0}\right)$ is finite, condition (**) holds, and $P \in \mathcal{H}, P \leq Q \leq P^{\bullet}$ imply $Q \in \mathcal{H}$. Then for each $P, \widehat{P} \in \mathcal{P}$ such that $\widehat{P}$ is fully normalized in $\mathcal{F}$, there is a morphism $\varphi \in \operatorname{Hom}_{\mathcal{F}}\left(N_{S}(P), N_{S}(\widehat{P})\right)$ such that $\varphi(P)=\widehat{P}$.

Proof (a) For each proper $\mathcal{P}$-pair $(Q, P)$, define

$$
K_{P}=\{\varphi \in \operatorname{Aut}(Q) \mid \varphi(P)=P\} \leq \operatorname{Aut}(Q) .
$$

Notice that

$$
\begin{aligned}
\operatorname{Aut}_{S}^{K_{P}}(Q) & =\operatorname{Aut}_{S}(Q) \cap K_{P}=\operatorname{Aut}_{N_{S}(P)}(Q), \\
\operatorname{Aut}_{\mathcal{F}}^{K_{P}}(Q) & =\operatorname{Aut}_{\mathcal{F}}(Q) \cap K_{P}=\operatorname{Aut}_{N_{\mathcal{F}}(P)}(Q), \\
N_{S}^{K_{P}}(Q) & =N_{N_{S}(P)}(Q) .
\end{aligned}
$$

Assume the pair $(Q, P)$ is fully normalized. Fix $Q^{\prime} \in Q^{\mathcal{F}}$ and $\alpha \in \operatorname{Iso}_{\mathcal{F}}\left(Q, Q^{\prime}\right)$, and set $P^{\prime}=\alpha(P)$. Then $\left(Q^{\prime}, P^{\prime}\right) \in(Q, P)^{\mathcal{F}}$, and

$$
{ }^{\alpha} K_{P}=K_{P^{\prime}}:=\left\{\varphi \in \operatorname{Aut}\left(Q^{\prime}\right) \mid \varphi\left(P^{\prime}\right)=P^{\prime}\right\} .
$$

Hence by (4.2),

$$
\left|N_{S}^{K_{P}}(Q)\right|=\left|N_{N_{S}(P)}(Q)\right| \geq\left|N_{N_{S}\left(P^{\prime}\right)}\left(Q^{\prime}\right)\right|=\left|N_{S}^{K_{P^{\prime}}}\left(Q^{\prime}\right)\right|=\left|N_{S}^{\alpha} K_{P}\left(Q^{\prime}\right)\right|,
$$

and so $Q$ is fully $K_{P}$-normalized in $\mathcal{F}$. By Lemma 2.2(b) (and since $Q \in \mathcal{H}$ and $\mathcal{F}$ is $\mathcal{H}$-saturated), $Q$ is receptive and fully $K_{P}$-automized in $\mathcal{F}$. So by (4.1),

$$
\operatorname{Aut}_{N_{S}(P)}(Q) \in \operatorname{Syl}_{p}\left(\operatorname{Aut}_{N_{\mathcal{F}}(P)}(Q)\right) .
$$

(b) Let $(Q, P)$, and $\left(Q^{\prime}, P^{\prime}\right) \in(Q, P)^{\mathcal{F}}$, be proper $\mathcal{P}$-pairs such that $\left(Q^{\prime}, P^{\prime}\right)$ is fully normalized. Let $K_{P} \leq \operatorname{Aut}(Q)$ and $K_{P^{\prime}} \leq \operatorname{Aut}\left(Q^{\prime}\right)$ be as in (a). Since $\left(Q^{\prime}, P^{\prime}\right)$ is fully normalized, $Q^{\prime}$ is receptive and fully $K_{P^{\prime}}$-automized by (a).

Choose some $\psi \in \operatorname{Iso}_{\mathcal{F}}\left(Q, Q^{\prime}\right)$ such that $\psi(P)=P^{\prime}$. By Lemma 2.2(b), there are $\chi \in \operatorname{Aut}_{\mathcal{F}}^{K_{P^{\prime}}}\left(Q^{\prime}\right)$ and $\varphi \in \operatorname{Hom}_{\mathcal{F}}\left(N_{S}^{K_{P}}(Q), N_{S}^{K_{P^{\prime}}}\left(Q^{\prime}\right)\right)$ such that $\left.\varphi\right|_{Q}=\chi \circ \psi$. Then $\varphi(P)=\chi\left(P^{\prime}\right)=P^{\prime}, \varphi(Q)=Q^{\prime}$, and $N_{S}^{K_{P}}(Q)=N_{N_{S}(P)}(Q)$ and $N_{S}^{K_{P^{\prime}}}\left(Q^{\prime}\right)=$ $N_{N_{S}\left(P^{\prime}\right)}\left(Q^{\prime}\right)$ by (4.2). 
(c) Fix subgroups $P, \widehat{P} \in \mathcal{P}$ such that $\widehat{P}$ is fully normalized in $\mathcal{F}$. We will construct a morphism in $\mathcal{F}$ from $N_{S}(P)$ to $N_{S}(\widehat{P})$ which sends $P$ to $\widehat{P}$. In particular, $\widehat{P}$ will be fully centralized in $\mathcal{F}$, since its centralizer contains an injective image of the centralizer of any other subgroup in $\mathcal{P}$.

Let $\hat{\mathcal{T}}$ be the set of all sequences

$$
\xi=\left(P=P_{0}, Q_{0}, \varphi_{0} ; P_{1}, Q_{1}, \varphi_{1} ; \ldots ; P_{k-1}, Q_{k-1}, \varphi_{k-1} ; P_{k}=\widehat{P}\right)
$$

such that for all $0 \leq i \leq k-1,\left(Q_{i}, P_{i}\right)$ is a proper $\mathcal{P}$-pair, $\varphi_{i} \in \operatorname{Hom}_{\mathcal{F}}\left(Q_{i}, N_{S}\left(P_{i+1}\right)\right)$, and $\varphi_{i}\left(P_{i}\right)=P_{i+1}$. Let $\mathcal{T} \subseteq \hat{\mathcal{T}}$ be the subset of those sequences in which each $\varphi_{i}$ is maximal, in the sense that it cannot be extended in $\mathcal{F}$ to a subgroup of $N_{S}\left(P_{i}\right)$ which properly contains $Q_{i}$. We give $\hat{\mathcal{T}}$ the partial ordering by inclusion (of sequences of the same length with the same $P_{i}$ ); then $\mathcal{T}$ is the set of maximal elements in $\hat{\mathcal{T}}$. Since $\mathcal{F}$ is $\mathcal{H}$-saturated, axiom (III) ensures that each element of $\hat{\mathcal{T}}$ is contained in an element of $\mathcal{T}$.

We first check that $\mathcal{T} \neq \varnothing$. Choose any $\varphi \in \operatorname{Iso}_{\mathcal{F}}(P, \widehat{P})$. Since $\mathcal{F}$ is $\mathcal{H}$-generated, there are subgroups $P_{i}<R_{i} \in \mathcal{H}$ for $0 \leq i \leq k$, and morphisms $\phi_{i} \in \operatorname{Hom}_{\mathcal{F}}\left(R_{i}, R_{i+1}\right)$ for $i \leq k-1$, such that $P_{0}=P, P_{k}=\hat{P}, \phi_{i}\left(P_{i}\right)=P_{i+1}$, and $\varphi=\left.\phi_{k-1}\right|_{P_{k-1}} \circ \cdots \circ$ $\left.\left.\phi_{1}\right|_{P_{1}} \circ \phi_{0}\right|_{P_{0}}$. For each $i$, let $P_{i+1}=\phi_{i}\left(P_{i}\right)$. Then $N_{R_{i}}\left(P_{i}\right)>P_{i}$ by Lemma 1.12 (and since $\left.R_{i}>P_{i}\right)$. Set $Q_{i}=N_{R_{i}}\left(P_{i}\right)$ and $\varphi_{i}=\phi_{i} \mid Q_{i}$. Then $\left(Q_{i}, P_{i}\right)$ is a proper $\mathcal{P}$-pair, $\varphi_{i} \in \operatorname{Hom}_{\mathcal{F}}\left(Q_{i}, N_{S}\left(P_{i+1}\right)\right)$ and $\left(P_{0}, Q_{0}, \varphi_{0} ; P_{1}, Q_{1}, \varphi_{1} ; \ldots ; P_{k}\right) \in \hat{\mathcal{T}}$. Thus $\hat{\mathcal{T}} \neq \varnothing$, and $\mathcal{T} \neq \varnothing$ since each element is contained in a maximal element.

Lemma 3.5(a) applies in this situation by the extra hypotheses which were assumed. Hence for any $\xi \in \mathcal{T}$ as in (4.3), $\varphi_{i}$ extends to $\varphi_{i}^{\bullet} \in \operatorname{Hom}_{\mathcal{F}}\left(Q_{i}{ }^{\bullet}, N_{S}\left(P_{i+1}\right)^{\bullet}\right)$ for each $i$, and therefore to $Q_{i} \bullet \cap N_{S}\left(P_{i}\right)$. The maximality of $\varphi_{i}$ implies that

$$
Q_{i}=Q_{i}^{\bullet} \cap N_{S}\left(P_{i}\right)
$$

The same argument applied to $\varphi_{i}^{-1}$ shows that

$$
\varphi_{i}\left(Q_{i}\right)=\varphi_{i}\left(Q_{i}\right)^{\bullet} \cap N_{S}\left(P_{i+1}\right) .
$$

Let $\mathcal{T}_{r} \subseteq \mathcal{T}$ be the subset of those $\xi$ for which there is no $1 \leq i \leq k-1$ such that $Q_{i}=N_{S}\left(P_{i}\right)=\varphi_{i-1}\left(Q_{i-1}\right)$. Let

$$
\mathcal{T} \stackrel{R}{\longrightarrow} \mathcal{T}_{r}
$$

be the "reduction" map, which for every $1 \leq i \leq k-1$ such that $Q_{i}=N_{S}\left(P_{i}\right)=$ $\varphi_{i-1}\left(Q_{i-1}\right)$, removes from the sequence the triple $\left(P_{i}, Q_{i}, \varphi_{i}\right)$, and replaces $\varphi_{i-1}$ by the composite $\varphi_{i} \circ \varphi_{i-1}$. Since $\mathcal{T} \neq \varnothing$, the existence of the reduction map $R$ shows that $\mathcal{T}_{r} \neq \varnothing$. 
For each $\xi \in \mathcal{T}$, define

$$
I(\xi)=\left\{i=0, \ldots, k-1 \mid Q_{i}<N_{S}\left(P_{i}\right) \text { and } \varphi_{i}\left(Q_{i}\right)<N_{S}\left(P_{i+1}\right)\right\} .
$$

If $\xi \in \mathcal{T}$ and $I(\xi) \neq \varnothing$, define

$$
\lambda(\xi)=\min _{i \in I(\xi)}\left\{\left|Q_{i}^{\bullet}\right|\right\} \geq(0, p) .
$$

Since $\operatorname{Aut}_{\mathcal{F}}\left(S_{0}\right)$ is assumed to be finite, $\mathcal{F}^{\bullet}$ has finitely many $\mathcal{F}$-conjugacy classes by Lemma 3.2(c), so there are only a finite number of values that $\lambda(\xi)$ can take.

Assume there is a sequence $\xi \in \mathcal{T}_{r}$ as in (4.3) such that $I(\xi)=\varnothing$. For each $0 \leq i<k$, if $Q_{i}<N_{S}\left(P_{i}\right)$, then $\varphi_{i}\left(Q_{i}\right)=N_{S}\left(P_{i+1}\right)$ since $i \notin I(\xi)$, and hence $Q_{i+1}<N_{S}\left(P_{i+1}\right)$ if $i+1 \leq k-1$ since $\xi$ is reduced. Thus $\left|N_{S}\left(P_{i}\right)\right|>\left|Q_{i}\right|=$ $\left|N_{S}\left(P_{i+1}\right)\right|>\cdots>\left|N_{S}\left(P_{k}\right)\right|$ in this case, which contradicts the assumption that $P_{k}=\widehat{P}$ is fully normalized. Hence $Q_{i}=N_{S}\left(P_{i}\right)$ for each $i<k$, and $\varphi_{k-1} \circ \cdots \circ \varphi_{0}$ lies in $\operatorname{Hom}_{\mathcal{F}}\left(N_{S}(P), N_{S}(\widehat{P})\right)$ and sends $P$ to $\widehat{P}$.

It remains to show that there exists $\xi \in \mathcal{T}_{r}$ such that $I(\xi)=\varnothing$. To see this, fix $\xi \in \mathcal{T}_{r}$ as in (4.3) such that $I(\xi) \neq \varnothing$. We will construct $\hat{\xi} \in \mathcal{T}_{r}$ such that either $I(\hat{\xi})=\varnothing$ or $\lambda(\hat{\xi})>\lambda(\xi)$. Since $\lambda(\xi)$ can only take finitely many values, this will prove our claim.

Fix $i \in I(\xi)$, set $R_{i+1}=\varphi_{i}\left(Q_{i}\right)$, and choose a fully normalized proper $\mathcal{P}$-pair $\left(U_{i}, V_{i}\right)$ which is $\mathcal{F}$-conjugate to $\left(Q_{i}, P_{i}\right)$ (hence also to $\left.\left(R_{i+1}, P_{i+1}\right)\right)$. By (b), there are morphisms

$\psi_{i} \in \operatorname{Hom}_{\mathcal{F}}\left(N_{N_{S}\left(P_{i}\right)}\left(Q_{i}\right), N_{S}\left(V_{i}\right)\right) \quad$ and $\quad \theta_{i} \in \operatorname{Hom}_{\mathcal{F}}\left(N_{N_{S}\left(P_{i+1}\right)}\left(R_{i+1}\right), N_{S}\left(V_{i}\right)\right)$ such that $\psi_{i}\left(P_{i}\right)=\theta_{i}\left(P_{i+1}\right)=V_{i}$ and $\psi_{i}\left(Q_{i}\right)=\theta_{i}\left(R_{i+1}\right)=U_{i}$. Upon replacing $\left(P_{i}, Q_{i}, \varphi_{i}\right)$ by

$$
\left(P_{i}, N_{N_{S}\left(P_{i}\right)}\left(Q_{i}\right), \psi_{i} ; V_{i}, \theta_{i}\left(N_{N_{S}\left(P_{i+1}\right)}\left(R_{i+1}\right)\right), \theta_{i}^{-1}\right),
$$

we get a new sequence $\xi_{1} \in \hat{\mathcal{T}}$. Upon applying axiom (III) again, we get a sequence $\xi_{2} \in \mathcal{T}$ where $\left(P_{i}, Q_{i}, \varphi_{i}\right)$ has been replaced by

$$
\left(P_{i}, \widetilde{Q}_{i}, \widetilde{\psi}_{i} ; V_{i}, \widetilde{R}_{i+1}, \tilde{\theta}_{i}^{-1}\right)
$$

for some maximal extensions $\tilde{\psi}_{i}$ of $\psi_{i}$ and $\tilde{\theta}_{i}$ of $\theta_{i}$. Also, $Q_{i}<N_{S}\left(P_{i}\right)$ and $R_{i+1}<N_{S}\left(P_{i+1}\right)$ since $i \in I(\xi)$, so

$$
\widetilde{Q}_{i} \geq N_{N_{S}\left(P_{i}\right)}\left(Q_{i}\right)>Q_{i} \quad \text { and } \quad \widetilde{R}_{i+1} \geq N_{N_{S}\left(P_{i+1}\right)}\left(R_{i+1}\right)>R_{i+1},
$$

and ${\widetilde{\tilde{Q}_{i}}}^{\bullet}>Q_{i}{ }^{\bullet}$ and $\widetilde{R}_{i+1}{ }^{\bullet}>R_{i+1} \bullet$ by (4.4) and (4.5). In particular, $\left|\widetilde{Q}_{i}^{\bullet}\right|>\lambda(\xi)$ and $\left|\widetilde{R}_{i+1} \bullet\right|>\lambda(\xi)$. 
Upon repeating this procedure for all $i \in I(\xi)$, we obtain a new element $\xi^{\prime} \in \mathcal{T}$ such that either $I\left(\xi^{\prime}\right)=\varnothing$ or $\lambda\left(\xi^{\prime}\right)>\lambda(\xi)$. Set $\hat{\xi}=R\left(\xi^{\prime}\right) \in \mathcal{T}_{r}$. Then either $I(\hat{\xi})=\varnothing$ or $\lambda(\hat{\xi})>\lambda(\xi)$. Since the function $\lambda$ can only take a finite number of possible values, it follows by induction that there is $\xi \in \mathcal{T}_{r}$ such that $I(\xi)=\varnothing$.

Very roughly, Lemma 4.4 allows us to reduce the proof of Theorem 4.2 to showing that the saturation properties hold for certain subgroups that are normal in the fusion system. This case is handled by the following lemma.

Lemma 4.5 Let $\mathcal{F}$ be a fusion system over a discrete $p$-toral group $S$ such that $\operatorname{Aut}_{\mathcal{F}}\left(S_{0}\right)$ is finite and condition (*) holds. Fix a subgroup $Q \unlhd S$, set $\mathcal{H}=\{P \leq S \mid$ $P>Q\}$ and assume that:

(i) $Q \unlhd \mathcal{F}$.

(ii) $\mathcal{F}$ is $\mathcal{H}$-generated and $\mathcal{H}$-saturated.

(iii) Either $Q$ is not $\mathcal{F}$-centric, or $\operatorname{Out}_{S}(Q) \cap O_{p}\left(\operatorname{Out}_{\mathcal{F}}(Q)\right) \neq 1$.

Then $Q$ is fully automized and receptive in $\mathcal{F}$.

Proof When $\Gamma$ is a group containing a normal discrete $p$-torus $P$ of finite index, we let $O_{p}(\Gamma)$ be the inverse image in $\Gamma$ of the maximal normal $p$-subgroup $O_{p}(\Gamma / P)$ under the obvious projection. Equivalently, this is the largest normal discrete $p$-toral subgroup of $\Gamma$. Define

$$
\widehat{Q}=\left\{x \in S \mid c_{x} \in O_{p}\left(\operatorname{Aut}_{\mathcal{F}}(Q)\right)\right\} .
$$

Then $\widehat{Q} \unlhd S$ by definition. We claim that $\widehat{Q}$ is strongly closed in $\mathcal{F}$. Assume that $x \in \widehat{Q}$ is $\mathcal{F}$-conjugate to $y \in S$. Since $Q$ is normal in $\mathcal{F}$, there exists $\psi \in$ $\operatorname{Hom}_{\mathcal{F}}(\langle x, Q\rangle,\langle y, Q\rangle)$ which satisfies $\psi(Q)=Q$ and $\psi(x)=y$. In particular, $\psi \circ c_{x} \circ \psi^{-1}=c_{y}$. It follows that $y \in \widehat{Q}$, since $c_{x} \in O_{p}\left(\operatorname{Aut}_{\mathcal{F}}(Q)\right)$.

Note also that $Q \cdot C_{S}(Q) \unlhd \widehat{Q}$, and $\hat{Q} / Q \cdot C_{S}(Q) \cong \operatorname{Out}_{S}(Q) \cap O_{p}\left(\operatorname{Out}_{\mathcal{F}}(Q)\right)$. If $Q$ is $\mathcal{F}$-centric, then this last group is nontrivial by (iii), and if not, then $Q \cdot C_{S}(Q)>Q$ by definition. Thus $\widehat{Q}>Q$ in either case, and so $\widehat{Q} \in \mathcal{H}$.

Consider the statement

$$
\text { each } \varphi \in \operatorname{Aut}_{\mathcal{F}}(Q) \text { extends to some } \bar{\varphi} \in \operatorname{Aut}_{\mathcal{F}}(\hat{Q}) .
$$

We first prove that (4.6) implies the lemma, and then prove (4.6).

Point (4.6) implies the lemma Since $Q$ is normal in $\mathcal{F}$ and $\widehat{Q}$ is strongly closed, each of them is the only subgroup in its $\mathcal{F}$-conjugacy class. So $Q$ and $\widehat{Q}$ are both fully 
centralized and fully normalized in $\mathcal{F}$. Also, $\widehat{Q}$ is receptive and fully automized in $\mathcal{F}$, since $\mathcal{F}$ is $\mathcal{H}$-saturated and $\hat{Q}^{\mathcal{F}}=\{\widehat{Q}\} \subseteq \mathcal{H}$.

By (4.6), the restriction map from $\operatorname{Aut}_{\mathcal{F}}(\hat{Q})$ to $\operatorname{Aut}_{\mathcal{F}}(Q)$ is surjective, and so we have $\operatorname{Aut}_{S}(Q) \in \operatorname{Syl}_{p}\left(\operatorname{Aut}_{\mathcal{F}}(Q)\right)$ since $\operatorname{Aut}_{S}(\hat{Q}) \in \operatorname{Syl}_{p}\left(\operatorname{Aut}_{\mathcal{F}}(\hat{Q})\right)$. Thus $Q$ is fully automized in $\mathcal{F}$.

Next we prove that $Q$ is receptive in $\mathcal{F}$. Fix $\varphi \in \operatorname{Aut}_{\mathcal{F}}(Q)$. As usual, let $N_{\varphi}$ be the group of all $g \in N_{S}(Q)$ such that $\varphi c_{g} \varphi^{-1} \in \operatorname{Aut}_{S}(Q)$. By (4.6), $\varphi$ extends to some $\psi \in \operatorname{Aut}_{\mathcal{F}}(\hat{Q})$. Consider the groups of automorphisms

$$
\begin{aligned}
K & =\left\{\chi \in \operatorname{Aut}_{S}(\hat{Q})|\chi|_{Q}=c_{x} \text { some } x \in N_{\varphi}\right\}, \\
K_{0} & =\left\{\chi \in \operatorname{Aut}_{\mathcal{F}}(\hat{Q})|\chi|_{Q}=\operatorname{Id}_{Q}\right\} \unlhd \operatorname{Aut}_{\mathcal{F}}(\widehat{Q}) .
\end{aligned}
$$

By definition, for all $x \in N_{\varphi}$, we have $\left.\left(\psi c_{x} \psi^{-1}\right)\right|_{Q}=\left.\chi\right|_{Q}$ for some $\chi \in \operatorname{Aut}_{S}(\hat{Q})$. In other words, as subgroups of $\operatorname{Aut}(\hat{Q})$,

$$
{ }^{\psi} K \leq \operatorname{Aut}_{S}(\hat{Q}) \cdot K_{0} .
$$

Now, we have $\operatorname{Aut}_{S}(\hat{Q}) \in \operatorname{Syl}_{p}\left(\operatorname{Aut}_{S}(\hat{Q}) K_{0}\right)$ since $\hat{Q}$ is fully automized, so there are $\omega \in \operatorname{Aut}_{S}(\hat{Q})$ and $\chi \in K_{0}$ such that ${ }^{\omega \chi}\left({ }^{\psi} K\right) \leq \operatorname{Aut}_{S}(\hat{Q})$. Hence ${ }^{\psi}{ }^{\prime} K \leq \operatorname{Aut}_{S}(\hat{Q})$. Since $\widehat{Q}$ is receptive in $\mathcal{F}, \chi \psi \in \operatorname{Aut}_{\mathcal{F}}(\widehat{Q})$ extends to a morphism $\bar{\varphi}$ defined on $N_{\chi \psi} \geq N_{S}^{K}(\hat{Q}) \geq N_{\varphi}$, and $\left.\bar{\varphi}\right|_{Q}=\left.\psi\right|_{Q}=\varphi$ since $\left.\chi\right|_{Q}=\operatorname{Id}_{Q}$.

Proof of (4.6) Since $\mathcal{F}$ is $\mathcal{H}$-generated, each $\varphi \in \operatorname{Aut}_{\mathcal{F}}(Q)$ is a composite of automorphisms of $Q$ which extend to strictly larger subgroups. So it suffices to show (4.6) when $\varphi$ itself extends to some $P>Q$.

Let $\mathcal{X}$ be the set of all subgroups $P \in \mathcal{H}$ such that $\varphi=\left.\bar{\varphi}\right|_{Q}$ for some $\bar{\varphi} \in \operatorname{Hom}_{\mathcal{F}}(P, S)$. We are assuming that $\mathcal{X} \neq \varnothing$. We claim that

$$
P \in \mathcal{X} \Longrightarrow N_{\widehat{Q} P}(P) \in \mathcal{X}
$$

Assume this, and fix $P_{1} \in \mathcal{X}$. If $\left|\widehat{Q} P_{1} / P_{1}\right|<\infty$, then by repeated application of (4.7) and Lemma 1.12 , we get that $\hat{Q} \in \mathcal{X}$. If not, then $|\widehat{Q} / Q|=\infty$, and $\widehat{Q}_{0} Q / Q$ is a nontrivial discrete $p$-torus. Set

$$
P_{2}=N_{\widehat{Q}_{0} P_{1}}\left(P_{1}\right) \quad \text { and } \quad P_{3}=P_{2} \cap \widehat{Q}_{0} Q .
$$

Then $P_{2} \in \mathcal{X}$ by (4.7), $P_{1} \leq P_{2} \leq \hat{Q}_{0} P_{1}$, and $P_{2}>P_{1}$ if $P_{2} \nsucceq \hat{Q}_{0}$ by Lemma 1.12. So $P_{3}>Q$, and hence $P_{3} \in \mathcal{X}$. Now set $P_{4}=N \widehat{Q}\left(P_{3}\right)$, so $P_{4} \in \mathcal{X}$ by (4.7) again, $P_{4} \geq \widehat{Q}_{0} Q$ since $\hat{Q}_{0} Q / Q$ is abelian, hence $\left[\widehat{Q}: P_{4}\right]<\infty$, and $\hat{Q} \in \mathcal{X}$ by earlier remarks. Since $\widehat{Q} \in \mathcal{X}, \varphi$ extends to some $\widehat{\varphi} \in \operatorname{Hom}_{\mathcal{F}}(\widehat{Q}, S)$, and $\hat{\varphi}(\widehat{Q})=\widehat{Q}$ since $\widehat{Q}$ is strongly closed in $\mathcal{F}$. This shows that (4.7) implies (4.6). 
It remains to prove (4.7). Fix $P \in \mathcal{X} \subseteq \mathcal{H}$, and let $\tilde{\varphi} \in \operatorname{Iso}_{\mathcal{F}}\left(P, P_{2}\right)$ be an extension of $\varphi$. Choose some $P_{3} \in P^{\mathcal{F}}=P_{2}{ }^{\mathcal{F}}$ which is fully automized and receptive in $\mathcal{F}$. Notice that $P_{3}>Q$, since $Q \unlhd \mathcal{F}$ by (i). Let $\psi \in \operatorname{Iso}_{\mathcal{F}}\left(P_{2}, P_{3}\right)$ be any isomorphism. Upon replacing $\tilde{\varphi}$ by $\psi \circ \tilde{\varphi}$, we can arrange that $P_{2}$ be fully automized and receptive.

Consider the groups of automorphisms

$$
\begin{aligned}
L & =\left\{\chi \in \operatorname{Aut}_{\mathcal{F}}\left(P_{2}\right)|\chi|_{Q} \in O_{p}\left(\operatorname{Aut}_{\mathcal{F}}(Q)\right)\right\}, \\
L_{0} & =\left\{\chi \in \operatorname{Aut}_{\mathcal{F}}\left(P_{2}\right)|\chi|_{Q}=\operatorname{Id}_{Q}\right\} .
\end{aligned}
$$

Both $L$ and $L_{0}$ are normal subgroups of $\operatorname{Aut}_{\mathcal{F}}\left(P_{2}\right)$. Also, $L / L_{0}$ is a discrete $p-$ toral group, since there is a monomorphism $L / L_{0} \rightarrow O_{p}\left(\operatorname{Aut}_{\mathcal{F}}\left(P_{2}\right)\right)$. Since $P_{2}$ is fully automized, $\operatorname{Aut}_{S}\left(P_{2}\right) \in \operatorname{Syl}_{p}\left(\operatorname{Aut}_{\mathcal{F}}\left(P_{2}\right)\right)$, and hence $\operatorname{Aut}_{S}^{L}\left(P_{2}\right) \in \operatorname{Syl}_{p}(L)$ and $L=\operatorname{Aut}_{S}^{L}\left(P_{2}\right) L_{0}$.

Thus $P_{2}$ is fully $L$-automized in $\mathcal{F}$. By Lemma 2.2(b), there are $g \in N_{S}^{L}\left(P_{2}\right)=$ $N_{\hat{Q}}\left(P_{2}\right), \chi \in L_{0}$, and $\psi \in \operatorname{Hom}_{\mathcal{F}}\left(N_{S}^{L^{\varphi}}(P) P, N_{S}^{L}\left(P_{2}\right) P_{2}\right)$, such that $\left.\psi\right|_{P}=\left(c_{g} \circ \chi\right) \circ \varphi$. Also, $N_{S}^{L^{\varphi}}(P) P=N_{\hat{Q} P} P(P)$. Upon replacing $\psi$ by $c_{g}^{-1} \circ \psi$, we can assume that $g=1$. Then $\left.\psi\right|_{Q}=\left.\varphi\right|_{Q}$, and thus $N_{\widehat{Q} P}(P) \in \mathcal{X}$.

The following lemma combines Lemmas 4.4 and 4.5.

Lemma 4.6 Let $\mathcal{F}$ be a fusion system over a discrete $p$-toral group $S$ such that $\operatorname{Aut}_{\mathcal{F}}\left(S_{0}\right)$ is finite and condition $(* *)$ holds. Fix a family $\mathcal{H} \varsubsetneqq \operatorname{Sub}(S)$, and a subgroup $P \leq S$ which is maximal in $\operatorname{Sub}(S) \backslash \mathcal{H}$. Assume that:

(i) $\mathcal{H}$ is invariant under $\mathcal{F}$-conjugacy.

(ii) $\mathcal{H}$ is closed in $\operatorname{Sub}(S)$, and $\mathcal{F}$ is $\mathcal{H}$-generated and $\mathcal{H}$-saturated.

(iii) $P \in \mathcal{H}$ and $P \leq Q \leq P^{\bullet}$ imply $Q \in \mathcal{H}$.

(iv) Either $P$ is not $\mathcal{F}$-centric, or $\operatorname{Out}_{S}(P) \cap O_{p}\left(\operatorname{Out}_{\mathcal{F}}(P)\right) \neq 1$.

Then $\mathcal{F}$ is $\left(\mathcal{H} \cup P^{\mathcal{F}}\right)$-saturated.

Proof By assumption, all overgroups of subgroups in $P^{\mathcal{F}}$ are in $\mathcal{H}$. Since $P^{\mathcal{F}}$ contains only finitely many $S$-conjugacy classes by Lemma 4.1 , there is a subgroup $\hat{Q} \in P^{\mathcal{F}}$ which is fully normalized in $\mathcal{F}$. By Lemma 4.4(c), for each $Q \in P^{\mathcal{F}}$, there is $\chi_{Q} \in \operatorname{Hom}_{\mathcal{F}}\left(N_{S}(Q), N_{S}(\hat{Q})\right)$ such that $\chi_{Q}(Q)=\hat{Q}$. Let $\mathcal{N} \subseteq \mathcal{H}$ be the family of all subgroups of $N_{S}(\hat{Q})$ which strictly contain $\hat{Q}$. We claim that the normalizer system $N_{\mathcal{F}}(\hat{Q})$ is $\mathcal{N}$-saturated. 
For each $Q \in \mathcal{N}$, the pair $(Q, \hat{Q})$ is a proper $P^{\mathcal{F}}$-pair. Let $\left(Q^{\prime}, P^{\prime}\right)$ be a proper $P^{\mathcal{F}}$-pair which is $\mathcal{F}$-conjugate to $(Q, \widehat{Q})$ and fully normalized in $\mathcal{F}$. Then $\widehat{Q}=$ $\chi_{P^{\prime}}\left(P^{\prime}\right)<Q^{\prime \prime}:=\chi_{P^{\prime}}\left(Q^{\prime}\right) \leq N_{S}(\hat{Q})$, so $Q^{\prime \prime} \in \mathcal{N}$, and $\left(Q^{\prime \prime}, \hat{Q}\right)$ is a proper $P^{\mathcal{F}}$-pair. Furthermore, since $\left(Q^{\prime}, P^{\prime}\right)$ is fully normalized, and since $\chi_{P^{\prime}}$ is a monomorphism, $\left(Q^{\prime \prime}, \hat{Q}\right)$ is also fully normalized in $\mathcal{F}$. If $\alpha \in \operatorname{Iso}_{\mathcal{F}}\left(Q, Q^{\prime}\right)$ is such that $\alpha(\hat{Q})=P^{\prime}$, then $\left(\chi_{P^{\prime}} \mid Q^{\prime}\right) \circ \alpha$ is a morphism in $N_{\mathcal{F}}(\hat{Q})$ which sends $(Q, \hat{Q})$ onto $\left(Q^{\prime \prime}, \hat{Q}\right)$. Hence by Lemma 4.4(a), applied with $\mathcal{P}=P^{\mathcal{F}}$, the subgroup $Q^{\prime \prime}$ is receptive in $\mathcal{F}$ (hence in $N_{\mathcal{F}}(\hat{Q})$ ), and is fully automized in $N_{\mathcal{F}}(\hat{Q})$. This shows that every $Q \in \mathcal{N}$ is $N_{\mathcal{F}}(\hat{Q})$-conjugate to some $Q^{\prime \prime} \in \mathcal{N}$ which is fully automized and receptive in the normalizer fusion system. Axiom (III) holds for $N_{\mathcal{F}}(\hat{Q})$ with respect to the family $\mathcal{N}$, since $\mathcal{N}$ is closed under overgroups, $\mathcal{N} \subseteq \mathcal{H}$, and $\mathcal{F}$ is $\mathcal{H}$-saturated. Thus $N_{\mathcal{F}}(\widehat{Q})$ is $\mathcal{N}$-saturated.

Conjugation by $\left.\left(\chi_{P}\right)\right|_{P} \in \operatorname{Iso}_{\mathcal{F}}(P, \widehat{Q})$ sends $\operatorname{Aut}_{\mathcal{F}}(P)$ isomorphically to $\operatorname{Aut}_{\mathcal{F}}(\hat{Q})$ and $\operatorname{Aut}_{S}(P)$ into $\operatorname{Aut}_{S}(\hat{Q})$. So if $P$ and $\hat{Q}$ are not $\mathcal{F}$-centric, then point (iv) implies that $\operatorname{Out}_{S}(\hat{Q}) \cap O_{p}\left(\operatorname{Out}_{\mathcal{F}}(\hat{Q})\right) \neq 1$. Thus by Lemma $4.5, \hat{Q}$ is receptive and fully automized in $N_{\mathcal{F}}(\hat{Q})$. Hence $\widehat{Q}$ is fully automized in $\mathcal{F}$. It is receptive in $\mathcal{F}$ since for each $Q \in P^{\mathcal{F}}$, there is an $\mathcal{F}$-morphism which sends $Q$ onto $\hat{Q}$ and $N_{S}(Q)$ into $N_{S}(\widehat{Q})$.

Axiom (III) holds for $\mathcal{H} \cup P^{\mathcal{F}}$ since it holds for $\mathcal{H}$ and since no subgroup in $P^{\mathcal{F}}$ contains any subgroups in $\mathcal{H}$. Thus $\mathcal{F}$ is $\left(\mathcal{H} \cup P^{\mathcal{F}}\right)$-saturated.

We are now ready to prove Theorem 4.2. The inductive Lemma 4.6 would suffice to prove the theorem for fusion systems over finite $p$-groups. But for fusion systems over discrete $p$-toral groups, because our groups have infinite chains of subgroups, the results of Section 3 are needed to allow an induction proof.

Proof of Theorem 4.2 Let $\mathfrak{K}$ be the set of all closed families of subgroups $\mathcal{K} \subseteq \operatorname{Sub}(S)$ such that:

- $\mathcal{K} \supseteq \mathcal{H}$.

- $\mathcal{K}$ is invariant under $\mathcal{F}$-conjugacy.

- $P \in \mathcal{K}$ and $P \leq Q \leq P^{\bullet}$ implies $Q \in \mathcal{K}$.

- $\mathcal{F}$ is $\mathcal{K}$-saturated.

We must show that $\operatorname{Sub}(S) \in \mathfrak{K}$.

Assume otherwise, and choose $\mathcal{K}_{0} \in \mathfrak{K}$ for which $\mathcal{K}_{0} \cap \mathcal{F}^{\bullet}$ contains the largest possible number of $\mathcal{F}$-conjugacy classes. Set $\mathcal{K}_{1}=\left\{P \leq S \mid P^{\bullet} \in \mathcal{K}_{0}\right\}$. Then $\mathcal{K}_{1} \in \mathfrak{K}$ by Lemma 3.5(b). If $\mathcal{K}_{1} \varsubsetneqq \operatorname{Sub}(S)$, then $\mathcal{F}^{\bullet} \nsubseteq \mathcal{K}_{0}$; let $P$ be maximal among subgroups in $\in \mathcal{F}^{\bullet} \backslash \mathcal{K}_{0}$. Then $P$ is maximal among subgroups in $\operatorname{Sub}(S) \backslash \mathcal{K}_{1}, \mathcal{K}_{1} \cup P^{\mathcal{F}} \in \mathfrak{K}$ by Lemma 4.6, and this contradicts the maximality assumption on $\mathcal{K}_{0}$. 


\section{Extensions of $p$-local compact groups}

The main result in this section is a version of [16, Theorem 9] which describes how to extend a $p$-local compact group by a finite group. But before proving this, we need to show some of the basic properties of linking systems over discrete $p$-toral groups. All of the results in this section are generalizations of results in [16] or earlier papers about linking systems over finite $p$-groups.

We first look at automorphisms of fusion and linking systems. The definitions are the same as in the finite case (eg Andersen, the last author and Ventura [1, Definition 1.13]).

Definition 5.1 (a) For any fusion system over a discrete $p$-toral group $S$, an automorphism $\alpha$ of $S$ is fusion preserving if there is an automorphism $\hat{\alpha}$ of $\mathcal{F}$ which sends an object $P$ to $\alpha(P)$ and sends a morphism $\varphi$ to $\alpha \varphi \alpha^{-1}$ (after restricting $\alpha$ in the obvious way). Let $\operatorname{Aut}(S, \mathcal{F})$ be the group of fusion preserving automorphisms of $S$, and set $\operatorname{Out}(S, \mathcal{F})=\operatorname{Aut}(S, \mathcal{F}) / \operatorname{Aut}_{\mathcal{F}}(S)$.

(b) For any linking system $\mathcal{L}$ over a discrete $p$-toral group $S$, an automorphism of categories

$$
\alpha: \mathcal{L} \stackrel{\cong}{\longrightarrow} \mathcal{L}
$$

is isotypical if for each $P \in \mathrm{Ob}(\mathcal{L}), \alpha\left(\delta_{P}(P)\right)=\delta_{\alpha(P)}(\alpha(P))$. Let $\operatorname{Aut}_{\text {typ }}^{I}(\mathcal{L})$ be the group of isotypical automorphisms of $\mathcal{L}$ which send inclusions to inclusions.

(c) Each $\gamma \in \operatorname{Aut}_{\mathcal{L}}(S)$ acts on the set $\operatorname{Mor}(\mathcal{L})$ by composing on the left or right with $\gamma$ and its restrictions. More precisely, for any $\varphi \in \operatorname{Mor}_{\mathcal{L}}(P, Q)$, set

$$
\begin{aligned}
& \gamma \varphi=\left.\gamma\right|_{Q, \pi(\gamma)(Q)} \circ \varphi \in \operatorname{Mor}_{\mathcal{L}}(P, \pi(\gamma)(Q)), \\
& \varphi \gamma=\left.\varphi \circ \gamma\right|_{\pi(\gamma)^{-1}(P), P} \in \operatorname{Mor}_{\mathcal{L}}\left(\pi(\gamma)^{-1}(P), Q\right) \text {. }
\end{aligned}
$$

(d) For each $\gamma \in \operatorname{Aut}_{\mathcal{L}}(S)$, let $c_{\gamma} \in \operatorname{Aut}_{\text {typ }}^{I}(\mathcal{L})$ be the automorphism which sends an object $P$ to $\pi(\gamma)(P)$, and a morphism $\varphi$ to $\gamma \varphi \gamma^{-1}$. Set

$$
\operatorname{Out}_{\text {typ }}(\mathcal{L})=\operatorname{Aut}_{\text {typ }}^{I}(\mathcal{L}) /\left\{c_{\gamma} \mid \gamma \in \operatorname{Aut}_{\mathcal{L}}(S)\right\}
$$

The argument that $\operatorname{Aut}_{\text {typ }}^{I}(\mathcal{L})$ is a group in this situation is exactly the same as that used in [1, Lemma 1.14] when $S$ is finite.

Proposition 5.2 Let $(S, \mathcal{F}, \mathcal{L})$ be a linking triple, with structure functors

$$
\mathcal{T}_{\mathrm{Ob}(\mathcal{L})}(S) \stackrel{\delta}{\longrightarrow} \mathcal{L} \stackrel{\pi}{\longrightarrow} \mathcal{F} .
$$


Fix $\alpha \in \operatorname{Aut}_{\text {typ }}^{I}(\mathcal{L})$. Let $\beta \in \operatorname{Aut}(S)$ be the restriction of $\alpha$ to $S$ under the identification $S \cong \delta_{S}(S)$; thus $\alpha\left(\delta_{S}(g)\right)=\delta_{S}(\beta(g))$ for all $g \in S$. Then $\beta \in \operatorname{Aut}(S, \mathcal{F})$. Furthermore, $\pi \circ \alpha=\hat{\beta} \circ \pi$, where $\hat{\beta} \in \operatorname{Aut}(\mathcal{F})$ is the automorphism which sends $P$ to $\beta(P)$ and $\varphi$ to $\beta \varphi \beta^{-1}$.

Proof Clearly, $\alpha(S)=S$, and hence $\alpha$ sends $\delta_{S}(S)$ to itself. Thus $\beta$ is well-defined. For each $P \in \mathrm{Ob}(\mathcal{L})$ and $g \in P$, since $\alpha$ sends inclusions to inclusions, it sends

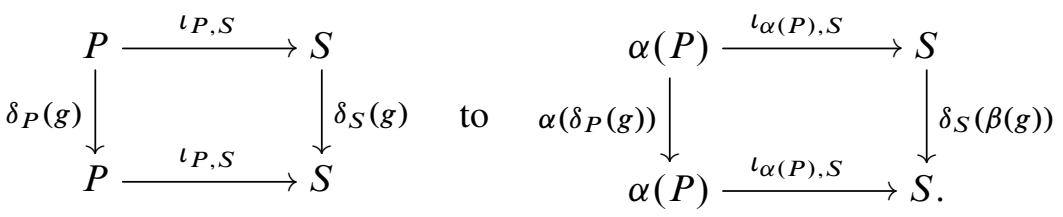

The first square commutes by axiom (C) in Definition 1.9, so the second also commutes. Since restrictions in $\mathcal{L}$ are uniquely defined by Proposition A.4(d), this shows that $\alpha\left(\delta_{P}(g)\right)=\left.\delta_{S}(\beta(g))\right|_{\alpha(P)}=\delta_{\alpha(P)}(\beta(g))$. Hence

$$
\delta_{\alpha(P)}(\beta(P))=\alpha\left(\delta_{P}(P)\right)=\delta_{\alpha(P)}(\alpha(P)),
$$

where the second equality holds since $\alpha$ is isotypical. Thus $\alpha(P)=\beta(P)$ since $\delta_{\alpha(P)}$ is injective.

Fix $P, Q \in \operatorname{Ob}(\mathcal{L})$ and $\psi \in \operatorname{Mor}_{\mathcal{L}}(P, Q)$, and set $\varphi=\pi(\psi) \in \operatorname{Hom}_{\mathcal{F}}(P, Q)$. For each $g \in P, \alpha$ sends

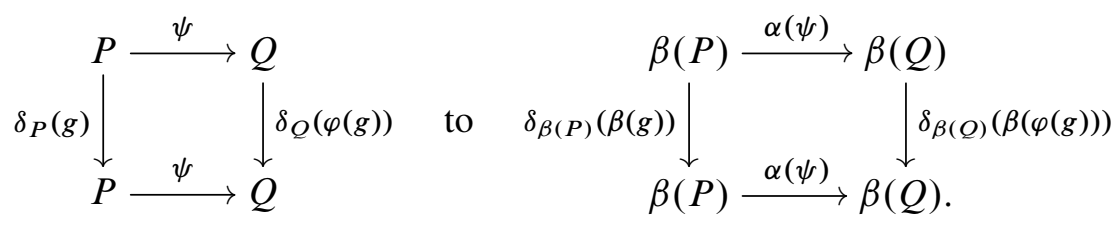

The first square commutes by axiom $(\mathrm{C})$, so the second also commutes. By $(\mathrm{C})$ again, $\delta_{\beta(Q)}(\beta(\varphi(g)))$ can be replaced by $\delta_{\beta(Q)}(\pi(\alpha(\psi))(\beta(g)))$, leaving the second square commutative. Since $\delta_{\beta(Q)}$ is a monomorphism, and since morphisms in $\mathcal{L}$ are epimorphisms by Proposition A.4(g), it follows that $\beta(\varphi(g))=\pi(\alpha(\psi))(\beta(g))$. Therefore

$$
\pi(\alpha(\psi))=\beta \varphi \beta^{-1}=\beta \pi(\psi) \beta^{-1} .
$$

So $\beta \varphi \beta^{-1} \in \operatorname{Hom}_{\mathcal{F}}(\beta(P), \beta(Q))$ for each $P, Q \in \operatorname{Ob}(\mathcal{L})$ and $\varphi \in \operatorname{Hom}_{\mathcal{F}}(P, Q)$. Since $\operatorname{Ob}(\mathcal{L})$ includes all subgroups which are $\mathcal{F}$-centric and $\mathcal{F}$-radical, all morphisms in $\mathcal{F}$ are composites of restrictions of morphisms between objects of $\mathcal{L}$ by Alperin's 
fusion theorem in the version of [9, Theorem 3.6]. Hence $\beta \varphi \beta^{-1} \in \operatorname{Mor}(\mathcal{F})$ for all $\varphi \in \operatorname{Mor}(\mathcal{F})$, and $\beta \in \operatorname{Aut}(S, \mathcal{F})$.

Thus there is a well-defined functor $\hat{\beta}$ from $\mathcal{F}$ to itself which sends each $P \leq S$ to $\beta(P)$ and sends each $\varphi \in \operatorname{Hom}_{\mathcal{F}}(P, Q)$ to $\beta \varphi \beta^{-1}$. This is an automorphism of the category $\mathcal{F}$ by the same argument applied to $\alpha^{-1}$, and $\pi \circ \alpha=\widehat{\beta} \circ \pi$ by (5.3).

We are now ready to define the structures which will be needed to construct extensions of linking systems.

Definition 5.3 Fix a linking triple $(S, \mathcal{F}, \mathcal{L})$ and a finite group $G$.

(a) An extension pair for $\mathcal{L}$ and $G$ is a pair $(\widehat{\Gamma}, \tau)$, where $\hat{\Gamma}$ is an extension of $\Gamma:=\operatorname{Aut}_{\mathcal{L}}(S) \unlhd \hat{\Gamma}$ by $G$, and where $\tau: \hat{\Gamma} \rightarrow \operatorname{Aut}_{\text {typ }}^{I}(\mathcal{L})$ is a homomorphism which makes both triangles in the following diagram commute:

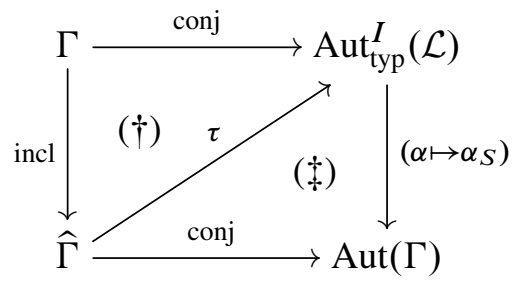

Fix an extension pair $U=(\widehat{\Gamma}, \tau)$ for $\mathcal{L}$ and $G$. Let $\rho: \hat{\Gamma} \rightarrow G$ be the surjection with kernel $\Gamma$.

(b) Let $\mathcal{L}_{U}=\mathcal{L}_{(\widehat{\Gamma}, \tau)}$ be the category with $\mathrm{Ob}\left(\mathcal{L}_{U}\right)=\operatorname{Ob}(\mathcal{L})$, and with

$$
\operatorname{Mor}\left(\mathcal{L}_{U}\right)=\operatorname{Mor}(\mathcal{L}) \times_{\Gamma} \hat{\Gamma}=(\operatorname{Mor}(\mathcal{L}) \times \hat{\Gamma}) / \sim,
$$

where $(\varphi, \gamma) \sim\left(\varphi^{\prime}, \gamma^{\prime}\right)$ if and only if there is $\lambda \in \Gamma$ such that $\varphi^{\prime}=\varphi \lambda$ and $\gamma^{\prime}=\lambda^{-1} \gamma$. Here, $\varphi \lambda$ is as defined in (5.2). When $\varphi \in \operatorname{Mor}_{\mathcal{L}}(\gamma(P), Q)$, the equivalence class of $(\varphi, \gamma)$ is denoted $\llbracket \varphi, \gamma \rrbracket \in \operatorname{Mor}_{\mathcal{L}_{U}}(P, Q)$. Composition in $\mathcal{L}_{U}$ is defined by

$$
\llbracket \psi, \eta \rrbracket \circ \llbracket \varphi, \gamma \rrbracket=\llbracket \psi \circ \tau(\eta)(\varphi), \eta \gamma \rrbracket .
$$

Here, $\tau(\eta)(\varphi) \in \operatorname{Mor}_{\mathcal{L}}(\eta \gamma(P), \eta(Q))$ when $\varphi \in \operatorname{Mor}_{\mathcal{L}}(\gamma(P), Q)$ (where we write $\eta(P)=\tau(\eta)(P)$, etc. $)$.

Thus when $U=(\widehat{\Gamma}, \tau)$ is an extension pair for $\mathcal{L}$ and $G, \llbracket \varphi \lambda, \gamma \rrbracket=\llbracket \varphi, \lambda \gamma \rrbracket$ in $\operatorname{Mor}\left(\mathcal{L}_{U}\right)$ for all $\varphi \in \operatorname{Mor}(\mathcal{L}), \lambda \in \Gamma$, and $\gamma \in \hat{\Gamma}$. 
To show composition in $\mathcal{L}_{U}$ is well defined, we note that for all $\psi, \varphi \in \operatorname{Mor}(\mathcal{L})$, $\mu, \lambda \in \Gamma$, and $\eta, \gamma \in \widehat{\Gamma}$ with appropriate domain and range,

$$
\begin{aligned}
\llbracket \psi \mu, \eta \rrbracket \circ \llbracket \varphi \lambda, \gamma \rrbracket & =\llbracket \psi \mu \circ \tau(\eta)(\varphi \lambda), \eta \gamma \rrbracket \\
& =\llbracket \psi \mu \circ \tau(\eta)(\varphi),\left(\eta \lambda \eta^{-1}\right) \eta \gamma \rrbracket \\
& =\llbracket \psi \mu \circ \tau(\eta)(\varphi) \mu^{-1}, \mu \eta \lambda \gamma \rrbracket \\
& =\llbracket \psi \circ \tau(\mu \eta)(\varphi), \mu \eta \lambda \gamma \rrbracket=\llbracket \psi, \mu \eta \rrbracket \circ \llbracket \varphi, \lambda \gamma \rrbracket .
\end{aligned}
$$

The second equality follows from the commutativity of triangle ( $\$)$ in Definition 5.3, and the fourth from that of $(\dagger)$.

We are now ready to state and prove the main result of this section. The following theorem is a generalization to $p$-local compact groups of [16, Theorem 9] (which in turn was a generalization of [6, Theorem 4.6]).

Theorem 5.4 [16, Theorem 9] Fix a linking triple $(\bar{S}, \overline{\mathcal{F}}, \overline{\mathcal{L}})$. Set $\overline{\mathcal{H}}=\operatorname{Ob}(\overline{\mathcal{L}})$, and assume it is closed under overgroups. Let

$$
\mathcal{T}_{\overline{\mathcal{H}}}(\bar{S}) \stackrel{\bar{\delta}}{\longrightarrow} \overline{\mathcal{L}} \stackrel{\bar{\pi}}{\longrightarrow} \overline{\mathcal{F}}
$$

be the structure functors for $\overline{\mathcal{L}}$. Set $\bar{\Gamma}=\operatorname{Aut}_{\overline{\mathcal{L}}}(\bar{S})$, and regard $\bar{S}$ as a subgroup of $\bar{\Gamma}$ via the inclusion $\bar{\delta}: \mathcal{T}_{\overline{\mathcal{H}}}(\bar{S}) \rightarrow \overline{\mathcal{L}}$. Fix a finite group $G$ and an extension pair $U=(\Gamma, \tau)$ for $\overline{\mathcal{L}}$ and $G$, and choose $S \in \operatorname{Syl}_{p}(\Gamma)$. Then there is a saturated fusion system $\mathcal{F}$ over $S$ containing $\overline{\mathcal{F}}$, and a transporter system $\mathcal{T}$ associated to $\mathcal{F}$ and containing $\overline{\mathcal{L}}$, such that the following hold.

(a) We have $\operatorname{Ob}(\mathcal{T})=\mathcal{H}:=\{P \leq S \mid P \cap \bar{S} \in \overline{\mathcal{H}}\}$, and this set contains all subgroups of $S$ which are $\mathcal{F}$-centric and $\mathcal{F}$-radical. Also, $\mathcal{T}$ contains $\mathcal{L}_{U}$ as a full subcategory.

(b) The group $\Gamma$ can be identified with $\operatorname{Aut}_{\mathcal{T}}(\bar{S})$ in a way so that the inclusion of $\overline{\mathcal{L}}$ in $\mathcal{T}$ induces the inclusion of $\bar{\Gamma}=\operatorname{Aut}_{\overline{\mathcal{L}}}(\bar{S})$ in $\Gamma$.

(c) For each $\gamma \in \Gamma, c_{\gamma}=\tau(\gamma) \in \operatorname{Aut}_{\text {typ }}^{I}(\overline{\mathcal{L}})$.

(d) The subcategory $\overline{\mathcal{L}}$ is normal in $\mathcal{T}$ (cf Definition A.7).

(e) The space $\left|\mathcal{L}_{U}\right|$ is a deformation retract of $|\mathcal{T}|$. The inclusion of geometric realizations $|\overline{\mathcal{L}}| \subseteq\left|\mathcal{L}_{U}\right|(\simeq|\mathcal{T}|)$ is homotopy equivalent to a regular covering space $X \rightarrow|\mathcal{T}|$ with group of deck transformations $G \cong \Gamma / \bar{\Gamma}$.

Proof The categories $\mathcal{T}$ and $\mathcal{F}$ will be constructed in Step 2, after preliminary constructions in Step 1. We show that $\mathcal{T}$ is a transporter system in Steps 3 and 4, and prove that $\mathcal{F}$ is saturated in Step 5. Finally we prove (d) and (e) in Step 6. 
Note that $\bar{S}=O_{p}(\bar{\Gamma}) \in \operatorname{Syl}_{p}(\bar{\Gamma})$, since $\bar{\Gamma} / \bar{S}=\operatorname{Aut}_{\overline{\mathcal{L}}}(\bar{S}) / \bar{\delta}_{\bar{S}}(\bar{S})$ has order prime to $p$ by Proposition A.4(e). For each $\gamma \in \Gamma$, let $c_{\gamma} \in \operatorname{Aut}(\bar{S})$ denote conjugation by $\gamma$ on $\bar{S}=O_{p}(\bar{\Gamma}) \unlhd \Gamma$. By the commutativity of triangle $(\dagger)$ in Definition 5.3(a), this is the restriction to $\bar{S}$ of $\tau(\gamma)_{\bar{S}} \in \operatorname{Aut}(\bar{\Gamma})$. Hence by Proposition 5.2, $c_{\gamma}$ is fusion preserving (induces an automorphism of the category $\overline{\mathcal{F}}$ ), and $\tau(\gamma)(P)=c_{\gamma}(P)$ for all $P \in \overline{\mathcal{H}}$. To simplify notation below, we write $\gamma(P)=\tau(\gamma)(P)$ to denote this action of $\gamma$ on $\overline{\mathcal{H}}$.

Step 1 Set $\mathcal{L}_{1}=\mathcal{L}_{U}$. Thus by Definition $5.3, \operatorname{Ob}\left(\mathcal{L}_{1}\right)=\operatorname{Ob}(\overline{\mathcal{L}})=\overline{\mathcal{H}}$, and

$$
\operatorname{Mor}\left(\mathcal{L}_{1}\right)=\operatorname{Mor}(\overline{\mathcal{L}}) \times_{\bar{\Gamma}} \Gamma=\{\llbracket \varphi, \gamma \rrbracket \mid \varphi \in \operatorname{Mor}(\overline{\mathcal{L}}), \gamma \in \Gamma\} .
$$

We claim that

$$
\text { all morphisms in } \mathcal{L}_{1} \text { are monomorphisms and epimorphisms. }
$$

For any $\llbracket \varphi, \gamma \rrbracket, \llbracket \varphi^{\prime}, \gamma^{\prime} \rrbracket$, and $\llbracket \psi, \eta \rrbracket$ with appropriate domain and range,

$\llbracket \psi, \eta \rrbracket \circ \llbracket \varphi, \gamma \rrbracket=\llbracket \psi, \eta \rrbracket \circ \llbracket \varphi^{\prime}, \gamma^{\prime} \rrbracket$

$\Longrightarrow \llbracket \psi \circ \tau(\eta)(\varphi), \eta \gamma \rrbracket=\llbracket \psi \circ \tau(\eta)\left(\varphi^{\prime}\right), \eta \gamma^{\prime} \rrbracket$

$\Longrightarrow$ there exists $\lambda \in \bar{\Gamma}, \eta \gamma=\lambda^{-1} \eta \gamma^{\prime}$ and $\psi \circ \tau(\eta)(\varphi)=\psi \circ \tau(\eta)\left(\varphi^{\prime}\right) \circ \lambda$

$\Longrightarrow \gamma=\left(\eta^{-1} \lambda \eta\right)^{-1} \gamma^{\prime}$, and $\varphi=\varphi^{\prime} \circ \tau\left(\eta^{-1}\right)(\lambda)$,

where the second equality in the last line holds since morphisms in $\overline{\mathcal{L}}$ are monomorphisms (Proposition A.4(g)). Also, $\tau\left(\eta^{-1}\right)(\lambda)=\eta^{-1} \lambda \eta$ by the commutativity of $(\ddagger)$, so $\llbracket \varphi, \gamma \rrbracket=\llbracket \varphi^{\prime}, \gamma^{\prime} \rrbracket$, and hence $\llbracket \psi, \eta \rrbracket$ is a monomorphism. The proof that morphisms are epimorphisms is similar.

Set $\operatorname{Aut}_{\Gamma}(\bar{S})=\left\{c_{\gamma} \in \operatorname{Aut}(\bar{S}) \mid \gamma \in \Gamma\right\}$. Let $\mathcal{F}_{1}$ be the smallest fusion system over $\bar{S}$ which contains $\overline{\mathcal{F}}$ and $\operatorname{Aut}_{\Gamma}(\bar{S})$. Define

$$
\pi_{1}: \mathcal{L}_{1} \longrightarrow \mathcal{F}_{1}
$$

to be the identity on objects, while setting $\pi_{1}(\llbracket \varphi, \gamma \rrbracket)=\bar{\pi}(\varphi) \circ c_{\gamma}$. The proof that this is a functor (ie that it preserves composition) reduces to showing that the square

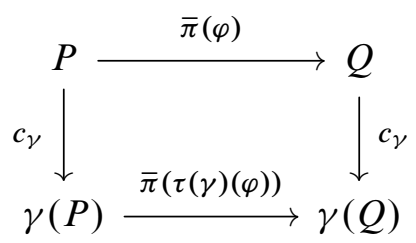

commutes for each $\varphi \in \operatorname{Mor}_{\overline{\mathcal{L}}}(P, Q)$ and each $\gamma \in \Gamma$. By the above remarks, $c_{\gamma} \in$ $\operatorname{Aut}(\bar{S}, \overline{\mathcal{F}})$ is the restriction to $\bar{S}$ of $\tau(\gamma)_{\bar{S}}$. By the last statement in Proposition 5.2, applied with $\alpha=\tau(\gamma)$ and $\beta=c_{\gamma}, \bar{\pi} \circ \tau(\gamma)=\widehat{c}_{\gamma} \circ \bar{\pi}$, where $\widehat{c}_{\gamma} \in \operatorname{Aut}(\overline{\mathcal{F}})$ is such 
that $\hat{c}_{\gamma}(\bar{\pi}(\varphi))=c_{\gamma} \bar{\pi}(\varphi) c_{\gamma}^{-1}$. Hence the square commutes. Since $\pi_{1}\left(\mathcal{L}_{1}\right)$ contains $\left.\overline{\mathcal{F}}\right|_{\overline{\mathcal{H}}}$ and $\operatorname{Aut}_{\bar{\Gamma}}(\bar{S})$, and is closed under restrictions of morphisms to subgroups in $\overline{\mathcal{H}}$ (Proposition A.4(d)), then $\pi_{1}$ maps onto $\left.\mathcal{F}_{1}\right|_{\overline{\mathcal{H}}}$.

We regard $\overline{\mathcal{L}}$ as a subcategory of $\mathcal{L}_{1}$ by identifying each morphism $\varphi \in \operatorname{Mor}_{\overline{\mathcal{L}}}(P, Q)$ with $\llbracket \varphi, 1 \rrbracket \in \operatorname{Mor}_{\mathcal{L}_{1}}(P, Q)$. By construction, $\bar{\pi}=\left.\pi_{1}\right|_{\overline{\mathcal{L}}}$. For $P \leq Q$ in $\overline{\mathcal{H}}$, the inclusion morphism $\iota_{P, Q}=\bar{\delta}_{P, Q}(1)$ for $\overline{\mathcal{L}}$ is also considered as an inclusion morphism in $\mathcal{L}_{1}$. The existence of restricted morphisms in $\overline{\mathcal{L}}$ (Proposition A.4(d)) carries over easily to the existence of restricted morphisms in $\mathcal{L}_{1}$, and they are unique by (5.4).

For all $P, Q \in \overline{\mathcal{H}}$, define

$$
\left(\delta_{1}\right)_{P, Q}: N_{S}(P, Q) \longrightarrow \operatorname{Mor}_{\mathcal{L}_{1}}(P, Q)
$$

by setting $\left(\delta_{1}\right)_{P, Q}(s)=\llbracket \iota_{s} P_{s^{-1}}, Q, s \rrbracket$. When $s \in N_{\bar{S}}(P, Q)$, we have $\llbracket \iota_{s} P_{s^{-1}}, Q, s \rrbracket=$ $\llbracket \bar{\delta}_{P, Q}(s), 1 \rrbracket$; and thus $\left(\delta_{1}\right)_{P, Q}$ extends the monomorphism $\bar{\delta}_{P, Q}$ from $N_{\bar{S}}(P, Q)$ to $\operatorname{Mor}_{\overline{\mathcal{L}}}(P, Q)$, under the identification of $\overline{\mathcal{L}}$ as a subcategory of $\mathcal{L}_{1}$. To simplify the notation, we write $\delta_{1}(x)=\left(\delta_{1}\right)_{P, Q}(x)$ when $P$ and $Q$ are understood.

We claim that for all $P, Q \in \overline{\mathcal{H}}, \psi \in \operatorname{Mor}_{\mathcal{L}_{1}}(P, Q)$, and $x \in P$,

$$
\left(\delta_{1}\right)_{Q}\left(\pi_{1}(\psi)(x)\right) \circ \psi=\psi \circ\left(\delta_{1}\right)_{P}(x) .
$$

Set $\psi=\llbracket \varphi, \gamma \rrbracket$, where $\gamma \in \Gamma$ and $\varphi \in \operatorname{Mor}_{\overline{\mathcal{L}}}(\gamma(P), Q)$. Then

$$
\begin{aligned}
\psi \circ \delta_{1}(x) & =\llbracket \varphi, \gamma \rrbracket \circ \llbracket \operatorname{Id}_{P}, x \rrbracket=\llbracket \varphi, \gamma x \rrbracket=\llbracket \varphi, c_{\gamma}(x) \gamma \rrbracket=\llbracket \varphi \circ \bar{\delta}\left(c_{\gamma}(x)\right), \gamma \rrbracket \\
& =\llbracket \bar{\delta}\left(\bar{\pi}(\varphi)\left(c_{\gamma}(x)\right)\right) \circ \varphi, \gamma \rrbracket=\llbracket \bar{\delta}\left(\pi_{1}(\psi)(x)\right) \circ \varphi, \gamma \rrbracket \\
& =\llbracket \bar{\delta}\left(\pi_{1}(\psi)(x)\right), 1 \rrbracket \circ \llbracket \varphi, \gamma \rrbracket=\delta_{1}\left(\pi_{1}(\psi)(x)\right) \circ \psi,
\end{aligned}
$$

where the fifth equality holds by axiom $(\mathrm{C})$ for the linking system $\overline{\mathcal{L}}$.

We next show that morphisms in $\mathcal{L}_{1}$ have the following extension property:

(5.5) For all $P, Q \in \overline{\mathcal{H}}, \psi \in \operatorname{Iso}_{\mathcal{L}_{1}}(P, Q)$ and $P^{\prime}, Q^{\prime} \leq \bar{S}$

$$
\text { for which } P \unlhd P^{\prime}, Q \unlhd Q^{\prime} \text { and } \psi \delta_{1}\left(P^{\prime}\right) \psi^{-1} \leq \delta_{1}\left(Q^{\prime}\right) \text {, }
$$

there is a unique $\psi^{\prime} \in \operatorname{Mor}_{\mathcal{L}_{1}}\left(P^{\prime}, Q^{\prime}\right)$, where $\left.\psi^{\prime}\right|_{P, Q}=\psi$.

Set $\psi=\llbracket \varphi, \gamma \rrbracket$, where $\varphi \in \operatorname{Mor}_{\overline{\mathcal{L}}}(\gamma(P), Q)$. For all $x \in P^{\prime}$,

$$
\begin{aligned}
\llbracket \varphi, \gamma \rrbracket \circ \llbracket \bar{\delta}(x), 1 \rrbracket \circ \llbracket \varphi, \gamma \rrbracket^{-1} & =\llbracket \varphi \circ \tau(\gamma)(\bar{\delta}(x)) \circ \varphi^{-1}, 1 \rrbracket \\
& =\llbracket \varphi \circ \bar{\delta}\left(c_{\gamma}(x)\right) \circ \varphi^{-1}, 1 \rrbracket \in \delta_{1}\left(Q^{\prime}\right),
\end{aligned}
$$

where $\tau(\gamma)(\bar{\delta}(x))=\bar{\delta}\left(c_{\gamma}(x)\right)$ by the commutativity of $(\$)$. Thus $\varphi \bar{\delta}\left(\gamma\left(P^{\prime}\right)\right) \varphi^{-1} \leq$ $\bar{\delta}\left(Q^{\prime}\right)$, so $\varphi$ extends to $\varphi^{\prime} \in \operatorname{Mor}_{\overline{\mathcal{L}}}\left(\gamma\left(P^{\prime}\right), Q^{\prime}\right)$ by Proposition A.4(f). Set $\psi^{\prime}=$ 
$\llbracket \varphi^{\prime}, \gamma \rrbracket$. Then $\left.\psi^{\prime}\right|_{P, Q}=\psi$ since $\tau(\gamma)\left(\iota_{P, P^{\prime}}\right)=\iota_{\gamma(P), \gamma\left(P^{\prime}\right)}$ (ie $\tau(\gamma)$ sends inclusions to inclusions), and this proves (5.5).

Step 2 Throughout the rest of the proof, for each $P \leq S$, we set $\bar{P}=P \cap \bar{S}$. We next construct categories $\mathcal{T}$ and $\mathcal{F}_{2}$, both of which have object sets $\mathcal{H}$, and which contain $\mathcal{L}_{1}$ and the restriction of $\mathcal{F}_{1}$ to $\overline{\mathcal{H}}$, respectively. Afterwards, we let $\mathcal{F}$ be the fusion system over $S$ generated by $\mathcal{F}_{2}$ and restrictions of morphisms.

Let $\mathcal{T}$ be the category with $\operatorname{Ob}(\mathcal{T})=\mathcal{H}$, and where for all $P, Q \in \mathcal{H}$,

$$
\operatorname{Mor}_{\mathcal{T}}(P, Q)=\left\{\psi \in \operatorname{Mor}_{\mathcal{L}_{1}}(\bar{P}, \bar{Q}) \mid \text { for all } x \in P \text {, there exists } y \in Q\right.
$$
such that $\left.\psi \circ \delta_{1}(x)=\delta_{1}(y) \circ \psi\right\}$.

If $\psi \in \operatorname{Mor}(\mathcal{T})$, then we denote the corresponding morphism in $\mathcal{L}_{1}$ by $\bar{\psi}$. Let

$$
\begin{aligned}
\delta_{P, Q}: N_{S}(P, Q) & \longrightarrow \operatorname{Mor}_{\mathcal{T}}(P, Q) \\
\subseteq N_{S}(\bar{P}, \bar{Q}) & \subseteq \operatorname{Mor}_{\mathcal{L}_{1}}(\bar{P}, \bar{Q})
\end{aligned}
$$

be the restriction of $\left(\delta_{1}\right) \bar{P}, \bar{Q}$. Let $\mathcal{F}_{2}$ be the category with $\operatorname{Ob}\left(\mathcal{F}_{2}\right)=\mathcal{H}$, and where $\operatorname{Mor}_{\mathcal{F}_{2}}(P, Q)=\left\{\varphi \in \operatorname{Hom}(P, Q) \mid\right.$ there exists $\psi \in \operatorname{Mor}_{\mathcal{L}_{1}}(\bar{P}, \bar{Q})$, where $\psi \circ \delta_{1}(x)=\delta_{1}(\varphi(x)) \circ \psi$, for all $\left.x \in P\right\}$.

Define $\pi: \mathcal{T} \rightarrow \mathcal{F}_{2}$ to be the identity on objects, and to send $\psi \in \operatorname{Mor}_{\mathcal{T}}(P, Q)$ to the homomorphism $\pi(\psi)(x)=y$ whenever $\psi \circ \delta_{1}(x)=\delta_{1}(y) \circ \psi$ (uniquely defined by (5.6) and (5.4)). This is clearly a functor: it is seen to preserve composition by juxtaposing the commutative squares which define $\pi$ on morphisms.

Let $\mathcal{F}$ be the fusion system over $S$ generated by $\mathcal{F}_{2}$ and restriction of homomorphisms. Since $\mathcal{H}=\operatorname{Ob}\left(\mathcal{F}_{2}\right)$ is closed under overgroups, $\mathcal{F}_{2}$ is a full subcategory of $\mathcal{F}$. Since $\mathcal{L}_{1}$ is a full subcategory of $\mathcal{T}, \operatorname{Hom}_{\mathcal{F}_{1}}(P, Q)=\operatorname{Hom}_{\mathcal{F}_{2}}(P, Q)$ for all $P, Q \in \overline{\mathcal{H}}$. If $P, Q \leq \bar{S}$ are any subgroups and $\varphi \in \operatorname{Hom}_{\mathcal{F}}(P, Q)$, then $\varphi$ is a composite of restrictions of morphisms in $\mathcal{F}_{2}$, and hence (since $P \in \mathrm{Ob}\left(\mathcal{F}_{2}\right)=\mathcal{H}$ implies $\bar{P} \in \overline{\mathcal{H}}$ ) a composite of restrictions of morphisms in $\mathcal{F}_{2}$ (equivalently $\mathcal{F}_{1}$ ) between subgroups in $\overline{\mathcal{H}}$. Thus $\varphi \in \operatorname{Hom}_{\mathcal{F}_{1}}(P, Q)$; and we conclude that $\mathcal{F}_{1}$ is also a full subcategory of $\mathcal{F}$.

Step 3 We next prove that

(5.7) each $P \in \mathcal{H}$ is $\mathcal{F}$-conjugate to some $P^{\prime} \in \mathcal{H}$

$$
\text { such that } \delta_{\bar{P}^{\prime}}\left(N_{S}\left(\bar{P}^{\prime}\right)\right) \in \operatorname{Syl}_{p}\left(\operatorname{Aut}_{\mathcal{T}}\left(\bar{P}^{\prime}\right)\right) \text {. }
$$

Fix $P \in \mathcal{H}$. Let $\mathcal{P}$ be the set of all $\bar{S}$-conjugacy classes $[\bar{Q}]$ of subgroups $\bar{Q} \in \bar{P}^{\overline{\mathcal{F}}}$ (recall $\bar{P}=P \cap \bar{S}$ ) which are fully normalized in $\overline{\mathcal{F}}$. (If $\bar{Q}$ is fully normalized in $\overline{\mathcal{F}}$, then so is every subgroup in $[\bar{Q}]$.) By Lemma $2.5,|\mathcal{P}|$ is finite and prime to $p$. 
We claim that in general, for each $\gamma \in \Gamma$ and each $\bar{Q}, \bar{R} \in \overline{\mathcal{H}}$,

$$
\begin{aligned}
& \bar{Q} \text { and } \bar{R} \overline{\mathcal{F}} \text {-conjugate } \Longleftrightarrow \gamma(\bar{Q}) \text { and } \gamma(\bar{R}) \overline{\mathcal{F}} \text {-conjugate, } \\
& \bar{Q} \text { and } \bar{R} \bar{S} \text {-conjugate } \Longleftrightarrow \gamma(\bar{Q}) \text { and } \gamma(\bar{R}) \bar{S} \text {-conjugate. }
\end{aligned}
$$

The first holds since $\gamma$ acts on $\overline{\mathcal{L}}$ and hence on $\overline{\mathcal{F}}$ as a group of automorphisms (Proposition 5.2), and the second since $\bar{S} \unlhd \Gamma$.

Let $\Gamma^{\prime} \subseteq \Gamma$ be the subset of those $\gamma \in \Gamma$ such that $\gamma(\bar{P}) \in \bar{P}^{\overline{\mathcal{F}}}$. Then for $\gamma_{1}$ and $\gamma_{2}$ in $\Gamma^{\prime}, \gamma_{1} \gamma_{2}(\bar{P}) \in \gamma_{1}(\bar{P})^{\overline{\mathcal{F}}}$ by (5.8) and since $\gamma_{2}(\bar{P}) \in \bar{P}^{\overline{\mathcal{F}}}$, and hence $\gamma_{1} \gamma_{2} \in \Gamma^{\prime}$. By (5.9) and since each $\gamma \in \Gamma$ acts on $\bar{S}$ via the fusion preserving automorphism $c_{\gamma} \in \operatorname{Aut}(\bar{S})$ as shown above, $\gamma$ permutes the $S$-conjugacy classes of subgroups which are fully normalized in $\overline{\mathcal{F}}$. Thus each element of $\Gamma^{\prime}$ permutes the set $\mathcal{P}$.

Fix $S^{\prime} \in \operatorname{Syl}_{p}\left(\Gamma^{\prime}\right)$. Let $\eta \in \Gamma$ be such that $S^{\prime \prime}:=\eta S^{\prime} \eta^{-1} \leq S$. Since $\mathcal{P}$ has order prime to $p$ by Lemma 2.4 , there is some $[\bar{Q}] \in \mathcal{P}$ fixed by $S^{\prime}$. In other words, for each $\gamma \in S^{\prime}, \gamma(\bar{Q})$ is $\bar{S}$-conjugate to $\bar{Q}$. So by (5.9), for each $s=\eta \gamma \eta^{-1} \in S^{\prime \prime}$ (where $\gamma \in S^{\prime}$ ), $s(\eta(\bar{Q})$ ) is $\bar{S}$-conjugate to $\eta(\bar{Q})$. Set $\bar{R}=\eta(\bar{Q})$. Then each coset in $S^{\prime \prime} / \bar{S}$ contains some element $s$ which normalizes $\bar{R}$, ie the obvious homomorphism $N_{S^{\prime \prime}}(\bar{R}) \rightarrow S^{\prime \prime} / \bar{S}$ is onto with kernel $N_{\bar{S}}(\bar{R})$. Since $S$ and $\bar{S}$ have the same identity component,

$$
\begin{aligned}
\left|\pi_{0}\left(N_{S}(\bar{R})\right)\right| \geq\left|\pi_{0}\left(N_{S^{\prime \prime}}(\bar{R})\right)\right| & =\left|\pi_{0}\left(N_{\bar{S}}(\bar{R})\right)\right| \cdot\left|S^{\prime \prime} / \bar{S}\right| \\
& =\left|\pi_{0}\left(N_{\bar{S}}(\bar{Q})\right)\right| \cdot\left|S^{\prime} / \bar{S}\right| .
\end{aligned}
$$

Since $\Gamma^{\prime}$ is the subgroup of elements of $\Gamma$ which send $\bar{P}$ to a subgroup in its $\overline{\mathcal{F}}-$ conjugacy class,

$$
\begin{aligned}
\left|\pi_{0}\left(\operatorname{Aut}_{\mathcal{T}}(\bar{R})\right)\right|=\left|\pi_{0}\left(\operatorname{Aut}_{\mathcal{T}}(\bar{P})\right)\right| & =\left|\pi_{0}\left(\operatorname{Aut}_{\overline{\mathcal{L}}}(\bar{P})\right)\right| \cdot\left|\Gamma^{\prime} / \bar{\Gamma}\right| \\
& =\left|\pi_{0}\left(\operatorname{Aut}_{\overline{\mathcal{L}}}(\bar{Q})\right)\right| \cdot\left|\Gamma^{\prime} / \bar{\Gamma}\right| .
\end{aligned}
$$

Since $\bar{Q}$ is fully normalized in $\overline{\mathcal{F}}, S^{\prime} \in \operatorname{Syl}_{p}\left(\Gamma^{\prime}\right)$, and $\bar{S} \in \operatorname{Syl}_{p}(\bar{\Gamma}),(5.10)$ and (5.11) imply that $\delta_{\bar{R}}\left(N_{S}(\bar{R})\right)$ is a Sylow $p$-subgroup of $\operatorname{Aut}_{\mathcal{T}}(\bar{R})$.

Choose any $\psi \in \operatorname{Iso}_{\mathcal{T}}(\bar{P}, \bar{R})$. Then $\psi \delta_{\bar{P}}\left(N_{S}(\bar{P})\right) \psi^{-1}$ is a $p$-subgroup of $\operatorname{Aut}_{\mathcal{T}}(\bar{R})$. Choose $\chi \in \operatorname{Aut}_{\mathcal{T}}(\bar{R})$ such that $(\chi \psi) \delta_{\bar{P}}\left(N_{S}(\bar{P})\right)(\chi \psi)^{-1} \leq \delta_{\bar{R}}\left(N_{S}(\bar{R})\right)$. By definition of the category $\mathcal{T}, \chi \psi$ extends to a morphism $\bar{\psi} \in \operatorname{Mor}_{\mathcal{T}}\left(P, N_{S}(\bar{R})\right)$. Set $P^{\prime}=\pi(\bar{\psi})(P)$. Then $\bar{P}^{\prime}=\bar{R}, P^{\prime}$ is $\mathcal{F}$-conjugate to $P$, and $P^{\prime} \in \mathcal{H}$ since $\bar{P}^{\prime} \in \overline{\mathcal{H}}$ ( $\overline{\mathcal{H}}$ is invariant under $\overline{\mathcal{F}}$-conjugacy). This finishes the proof of (5.7).

Step 4 We are now ready to show that $\mathcal{T}$ is a transporter system. For each $P \in \overline{\mathcal{H}}$, set

$$
E(P)=\operatorname{Ker}\left[\operatorname{Aut}_{\mathcal{T}}(P) \stackrel{\pi_{P}}{\longrightarrow} \operatorname{Aut}_{\mathcal{F}}(P)\right] .
$$


For each $P, Q \in \operatorname{Ob}(\mathcal{T}), E(P)$ acts on $\operatorname{Mor}_{\mathcal{T}}(P, Q)$ by right composition and $E(Q)$ by left composition. Both actions are free since all morphisms in $\mathcal{L}_{1}$ (hence in $\mathcal{T}$ ) are monomorphisms and epimorphisms by (5.4). We claim that $\pi_{P, Q}$ is the orbit map of the action of $E(P)$ on $\operatorname{Mor}_{\mathcal{T}}(P, Q)$. Since every morphism in $\mathcal{T}$ (and also by definition in $\mathcal{F}$ ) factors uniquely as the composite of an isomorphism followed by an inclusion, it suffices to prove this when $P$ and $Q$ are $\mathcal{F}$-conjugate. It thus suffices to prove it when $P=Q$, and this holds by definition of $E(P)$.

This proves axiom (A2). Axioms (A1) and (B) hold by construction, and (C) holds by definition of the functor $\pi: \mathcal{T} \rightarrow \mathcal{F}$. It remains to prove axioms (I)-(III).

Fix $P \in \mathcal{H}$ such that $\delta_{\bar{P}}\left(N_{S}(\bar{P})\right) \in \operatorname{Syl}_{p}\left(\operatorname{Aut}_{\mathcal{T}}(\bar{P})\right)$. By (5.7), every subgroup in $\mathcal{H}$ is $\mathcal{F}$-conjugate to some such $P$. Write $G=\operatorname{Aut}_{\mathcal{T}}(\bar{P}), T=\delta_{\bar{P}}\left(N_{S}(\bar{P})\right)$ and $P^{\prime}=\delta_{\bar{P}}(P)$ for short, where $\delta_{\bar{P}}$ is injective by construction. Thus $P^{\prime} \leq T \in \operatorname{Syl}_{p}(G)$. Fix $R \in \operatorname{Syl}_{p}\left(N_{G}\left(P^{\prime}\right)\right)$, and choose $\alpha \in G$ such that $\alpha R \alpha^{-1} \leq T$. Then $\alpha R \alpha^{-1} \in$ $\operatorname{Syl}_{p}\left(N_{G}\left(\alpha P^{\prime} \alpha^{-1}\right)\right)$, and so

$$
\alpha P^{\prime} \alpha^{-1} \leq \alpha R \alpha^{-1}=N_{T}\left(\alpha P^{\prime} \alpha^{-1}\right) .
$$

Set $Q=\delta_{\bar{P}}^{-1}\left(\alpha P^{\prime} \alpha^{-1}\right) \leq \delta_{\bar{P}}^{-1}(T)=N_{S}(\bar{P})$. Then $Q$ is $\mathcal{F}$-conjugate to $P, N_{S}(Q) \leq$ $N_{S}(\bar{P})$, and so

$$
\begin{aligned}
& N_{T}\left(\alpha P^{\prime} \alpha^{-1}\right)=N_{\delta_{\bar{P}}\left(N_{S}(\bar{P})\right)}\left(\delta_{\bar{P}}(Q)\right)=\delta_{\bar{P}}\left(N_{S}(Q)\right), \\
& N_{G}\left(\alpha P^{\prime} \alpha^{-1}\right)=N_{\operatorname{Aut}_{\mathcal{T}}(\bar{P})}\left(\delta_{\bar{P}}(Q)\right)=\operatorname{Aut}_{\mathcal{T}}(Q) .
\end{aligned}
$$

Thus $\delta_{Q}\left(N_{S}(Q)\right) \in \operatorname{Syl}_{p}\left(\operatorname{Aut}_{\mathcal{T}}(Q)\right)$, and this proves axiom (I).

We next claim that

$$
\begin{aligned}
P \unlhd P^{\prime} \leq S, Q \unlhd Q^{\prime} & \leq S, \psi \in \operatorname{Iso}_{\mathcal{T}}(P, Q), \psi\left(\delta_{P}\left(P^{\prime}\right)\right) \psi^{-1} \leq \delta_{Q}\left(Q^{\prime}\right) \\
& \Longrightarrow \text { there exists } \psi^{\prime} \in \operatorname{Mor}_{\mathcal{T}}\left(P^{\prime}, Q^{\prime}\right) \text { with }\left.\psi^{\prime}\right|_{P, Q}=\psi
\end{aligned}
$$

Set $\bar{\psi}=\left.\psi\right|_{\bar{P}}, \bar{Q}=\llbracket \varphi, \gamma \rrbracket$, where $\varphi \in \operatorname{Iso}_{\overline{\mathcal{L}}}(\gamma(\bar{P}), \bar{Q})$ and $\gamma \in \Gamma$. By Proposition A.4(f), $\varphi$ extends in a unique way to $\gamma\left(\bar{P}^{\prime}\right)$. Therefore, $\bar{\psi}$ extends to some unique $\bar{\psi}^{\prime} \in$ $\operatorname{Mor}_{\mathcal{L}_{1}}\left(\bar{P}^{\prime}, \bar{Q}^{\prime}\right)$. By definition of morphisms in $\mathcal{T}$ and the original hypothesis on $\psi, \bar{\psi}^{\prime}$ extends to a morphism $\psi^{\prime} \in \operatorname{Mor}_{\mathcal{T}}\left(P^{\prime}, Q^{\prime}\right)$ which extends $\psi$. This proves (5.12), and thus proves axiom (II).

It remains to prove axiom (III). Fix $P_{1} \leq P_{2} \leq P_{3} \leq \cdots$ in $\mathrm{Ob}(\mathcal{T})$ and $\psi_{i} \in$ $\operatorname{Mor}_{\mathcal{T}}\left(P_{i}, S\right)$ such that for all $i \geq 1$,

$$
\psi_{i}=\psi_{i+1} \circ \delta_{P_{i}, P_{i+1}}(1)
$$


Set $P=\bigcup_{i=1}^{\infty} P_{i}$. Let $\varphi_{i} \in \operatorname{Mor}(\overline{\mathcal{L}})$ and $\gamma_{i} \in \bar{\Gamma}$ be such that $\bar{\psi}_{i}=\llbracket \varphi_{i}, \gamma_{i} \rrbracket$ for each $i$. Since $|\Gamma / \bar{\Gamma}|<\infty$, we can assume, after passing to a subsequence, that the $\gamma_{i}$ all lie in the same coset $\bar{\Gamma} \gamma$. If $\gamma_{i}=v_{i} \gamma$ for some $v_{i} \in \bar{\Gamma}$, then $\bar{\psi}_{i}=\llbracket \varphi_{i} v_{i}, \gamma \rrbracket$; we can thus assume $\gamma_{i}=\gamma$ for each $i$. But then $\varphi_{i}=\varphi_{i+1} \mid P_{i}, P_{i+1}$ for each $i$, so by Proposition A.4(h) applied to $\overline{\mathcal{L}}$, there is $\varphi \in \operatorname{Mor}_{\mathcal{L}}(P, \bar{S})$ such that $\left.\varphi\right|_{P_{i}, \bar{S}}=\varphi_{i}$ for each $i$. Set $\bar{\psi}=\llbracket \varphi, \gamma \rrbracket \in \operatorname{Mor}_{\mathcal{T}}(\bar{P}, \bar{S})$; then $\left.\bar{\psi}\right|_{\bar{P}_{i}}=\bar{\psi}_{i}:=\left.\psi_{i}\right|_{\bar{P}_{i}}$ for each $i$.

Fix $g \in P$, and let $i$ be such that $g \in P_{i}$. Set $h=\pi\left(\psi_{i}\right)(g) \in S$. Then $\delta_{S}(h) \circ \psi_{i}=$ $\psi_{i} \circ \delta_{P_{i}}(g)$ by axiom (C) for $\mathcal{T}$, so $\left(\delta_{1}\right)_{\bar{S}}(h) \circ \bar{\psi}=\bar{\psi} \circ\left(\delta_{1}\right)_{\bar{P}_{i}}(g)$ by (5.4) (the morphisms are epimorphisms in $\left.\mathcal{L}_{1}\right)$. Hence by definition of $\mathcal{T}$, there is a unique morphism $\psi \in \operatorname{Mor}_{\mathcal{T}}(P, S)$ such that $\left.\psi\right|_{\bar{P}}=\bar{\psi}$. By the uniqueness of extensions again, $\left.\psi\right|_{P_{i}}=\psi_{i}$ for each $i$.

Step 5 We are now ready to show that $\mathcal{F}$ is saturated. By Theorem 4.2, it suffices to prove the following statements.

(i) The group $\operatorname{Aut}_{\mathcal{F}}\left(S_{0}\right)$ is finite.

(ii) For all $P \leq S, Q \leq S_{0}$, and $\varphi \in \operatorname{Hom}_{\mathcal{F}}(P, Q)$, there exists a morphism $\bar{\varphi} \in \operatorname{Hom}_{\mathcal{F}}\left(P \cdot C_{S}(P)_{0}, S_{0}\right)$ such that $\left.\bar{\varphi}\right|_{P}=\varphi$.

(iii) We have $\mathcal{H}$ is closed in $\operatorname{Sub}(S)$, and $\mathcal{F}$ is $\mathcal{H}$-generated and $\mathcal{H}$-saturated.

(iv) For all $P \in \mathcal{H}$ and $P \leq Q \leq P^{\bullet}, Q \in \mathcal{H}$.

(v) If $P \in \mathcal{F}^{\bullet}$ is $\mathcal{F}$-centric and $P \notin \mathcal{H}$, then there is $Q \in P^{\mathcal{F}}$ such that

$$
O_{p}\left(\operatorname{Out}_{\mathcal{F}}(Q)\right) \cap \operatorname{Out}_{S}(Q) \neq 1 .
$$

Point (i) holds by construction (and since $\overline{\mathcal{F}}$ is saturated). By Step 4 and Proposition A.3, we have $\mathcal{F}$ is $\mathcal{H}$-saturated; ie it satisfies the saturation axioms for subgroups in $\mathcal{H}$. It is also $\mathcal{H}$-generated by definition: each morphism in $\mathcal{F}$ is a composite of restrictions of morphisms between subgroups in $\mathcal{H}$. Since $\overline{\mathcal{H}}$ is closed under overgroups and $\overline{\mathcal{F}}$-conjugacy, $\mathcal{H}$ is closed under overgroups and $\mathcal{F}$-conjugacy by definition, and this finishes the proofs of (iii) and (iv).

If $P \leq S, Q \leq S_{0}$, and $\varphi \in \operatorname{Hom}_{\mathcal{F}}(P, Q)$, then $P \leq \bar{S}$ since $\bar{S}$ is strongly closed in $\mathcal{F}$ (and $\left.\bar{S}_{0}=S_{0}\right)$. By definition of $\mathcal{F}_{1}, \varphi=\chi \circ \psi$ for some $\chi \in \operatorname{Aut}_{\mathcal{F}}\left(S_{0}\right)\left(\chi=\left.\pi(\gamma)\right|_{S_{0}}\right.$ for some $\left.\gamma \in \Gamma=\operatorname{Aut}_{\mathcal{T}}(\bar{S})\right)$ and some $\psi \in \operatorname{Mor}(\overline{\mathcal{F}})$. Set $P^{\prime}=\psi(P) \leq S_{0}$, and choose $R \in P^{\overline{\mathcal{F}}}=P^{\prime \overline{\mathcal{F}}}$ which is receptive in $\overline{\mathcal{F}}$. By [9, Lemma 2.4(a)], $R \leq S_{0}$, and there is $w \in \operatorname{Aut}_{\overline{\mathcal{F}}}\left(S_{0}\right)$ such that $w\left(P^{\prime}\right)=R$. Since $R$ is receptive, $w \circ \psi$ extends to $\bar{\psi} \in \operatorname{Hom}_{\overline{\mathcal{F}}}\left(P \cdot C_{S}(P)_{0}, S_{0}\right)$, so $\varphi$ extends to $\chi \circ w^{-1} \circ \bar{\psi}$. This proves (ii).

It remains to prove (v). Let $\mathcal{K}$ be the set of all $P \in \mathcal{F}^{\bullet}$ such that the saturation axioms hold for subgroups $\mathcal{F}$-conjugate to $P$ and all of their overgroups. Since $\mathcal{H}$ is closed 
under overgroups and $\mathcal{F}$-conjugacy, $\mathcal{K} \supseteq \mathcal{H}^{\bullet}$. Set $\mathcal{K}^{\prime}=\mathcal{F}^{\bullet} \backslash \mathcal{K}$, and let $\overline{\mathcal{K}}^{\prime}$ be the set of all $\bar{P}=P \cap \bar{S}$ for $P \in \mathcal{K}^{\prime}$. We will show that for all $P \leq S$,

$$
P \in \mathcal{K} \text { or } \bar{P} \text { maximal in } \overline{\mathcal{K}}^{\prime} \Longrightarrow(\mathrm{v}) \text { holds for } P \text {. }
$$

Having done that, we will prove that $\mathcal{K}^{\prime}=\varnothing$, so $\mathcal{K} \supseteq \mathcal{F}^{\bullet}$, and hence (using (5.13)) that (v) holds for all $P \in \mathcal{F}^{\bullet}$.

We first show that

$$
P \in \mathcal{K} \text { or } \bar{P} \text { maximal in } \overline{\mathcal{K}}^{\prime}
$$

$\Longrightarrow$ there exists $Q \in P^{\mathcal{F}}$ such that $\bar{Q}$ is fully normalized in $\overline{\mathcal{F}}$.

If $\bar{P}$ is fully normalized, we are done, so assume otherwise. Let $\bar{P}^{\prime}$ be $\overline{\mathcal{F}}$-conjugate to $\bar{P}$ and fully normalized in $\overline{\mathcal{F}}$. Since $\overline{\mathcal{F}}$ is saturated, $\bar{P}^{\prime}$ is fully automized and receptive, and so by Lemma $1.7(\mathrm{c})$, there is $\rho \in \operatorname{Hom}_{\overline{\mathcal{F}}}\left(N_{\bar{S}}(\bar{P}), N_{\bar{S}}\left(\bar{P}^{\prime}\right)\right)$ such that $\rho(\bar{P})=\bar{P}^{\prime}$. Clearly, $\bar{P}<\bar{S}$, so $N_{\bar{S}}(\bar{P})>\bar{P}$. If $\bar{P}$ is maximal in $\overline{\mathcal{K}}^{\prime}$, then by Lemma 3.5(b), the saturation axioms hold for all $Q$ such that $\bar{Q}>\bar{P}$. So whether $\bar{P} \in \mathcal{K}$ or $\bar{P}$ is maximal in $\overline{\mathcal{K}}^{\prime}$, the saturation axioms hold for $N_{\bar{S}}(\bar{P}), N_{\bar{S}}\left(\bar{P}^{\prime}\right)$ and all subgroups of $S$ which contain them.

Set $R=N_{\bar{S}}(\bar{P})$, and let $K=\{\alpha \in \operatorname{Aut}(R) \mid \alpha(\bar{P})=\bar{P}\}$. Set $R^{\prime}=\rho(R)$ and $K^{\prime}={ }^{\rho} K$. Choose $R^{\prime \prime} \leq \bar{S}$ and $\tau \in \operatorname{Iso}_{\mathcal{F}}\left(R, R^{\prime \prime}\right)$ such that $R^{\prime \prime}$ is fully ${ }^{\tau} K$-normalized in $\mathcal{F}$, and set $\bar{P}^{\prime \prime}=\tau(\bar{P})$ and $K^{\prime \prime}={ }^{\tau} K$. Thus $K^{\prime}$ and $K^{\prime \prime}$ are the groups of automorphisms of $R^{\prime}$ and $R^{\prime \prime}$, respectively, which send $\bar{P}^{\prime}$ and $\bar{P}^{\prime \prime}$ to themselves. By Lemma 2.2(b), there are automorphisms $\chi, \chi^{\prime} \in \operatorname{Aut}_{\mathcal{F}}^{K^{\prime \prime}}\left(R^{\prime \prime}\right)$, and morphisms

$$
\bar{\tau} \in \operatorname{Hom}_{\mathcal{F}}\left(N_{S}^{K}(R), N_{S}^{K^{\prime \prime}}\left(R^{\prime \prime}\right)\right) \quad \text { and } \quad \bar{\rho} \in \operatorname{Hom}_{\mathcal{F}}\left(N_{S}^{K^{\prime}}\left(R^{\prime}\right), N_{S}^{K^{\prime \prime}}\left(R^{\prime \prime}\right)\right)
$$

such that $\left.\bar{\tau}\right|_{R}=\chi \tau$ and $\left.\bar{\rho}\right|_{R^{\prime}}=\chi^{\prime} \tau \rho^{-1}$.

We claim that

$$
\left|N_{\bar{S}}(\bar{P})\right|<\left|N_{\bar{S}}^{K^{\prime}}\left(R^{\prime}\right)\right| \leq\left|N_{\bar{S}}^{K^{\prime \prime}}\left(R^{\prime \prime}\right)\right| \leq\left|N_{\bar{S}}\left(\bar{P}^{\prime \prime}\right)\right|
$$

Since $\bar{P}$ is not fully normalized in $\overline{\mathcal{F}}, R^{\prime}=\rho\left(N_{\bar{S}}(\bar{P})\right)<N_{\bar{S}}\left(\bar{P}^{\prime}\right)$, and hence

$$
R^{\prime}<N_{N_{\bar{S}}\left(\bar{P}^{\prime}\right)}\left(R^{\prime}\right)=N_{\bar{S}}^{K^{\prime}}\left(R^{\prime}\right)
$$

by Lemma 1.12 . This proves the first inequality in (5.15). The next one holds since $\bar{\rho}$ sends

$$
N_{\bar{S}}^{K^{\prime}}\left(R^{\prime}\right) \text { into } N_{\bar{S}}^{K^{\prime \prime}}\left(R^{\prime \prime}\right)
$$

and the last holds since all elements of $N_{\bar{S}}^{K^{\prime \prime}}\left(R^{\prime \prime}\right)$ normalize $\overline{P^{\prime \prime}}$. Hence $P^{\prime \prime}$ is $\mathcal{F}_{-}$ conjugate to $P$ and

$$
\left|N_{\bar{S}}\left(\overline{P^{\prime \prime}}\right)\right|>\left|N_{\bar{S}}(\bar{P})\right|
$$


If $\bar{P}^{\prime \prime}$ is not fully normalized in $\overline{\mathcal{F}}$, then since $P^{\mathcal{F}}$ contains finitely many $S$-conjugacy classes (Lemma 4.1), we can repeat this procedure, until we find a subgroup $Q$ which satisfies (5.14).

We now prove (5.13). Assume $P$ is $\mathcal{F}$-centric and $P \notin \mathcal{H}$ (otherwise the statement is empty), and let $Q$ be $\mathcal{F}$-conjugate to $P$ such that $\bar{Q}$ is fully $\overline{\mathcal{F}}$-normalized. Thus $\bar{P} \notin \overline{\mathcal{H}}=\operatorname{Ob}(\overline{\mathcal{L}})$. Since by definition, a linking system must contain all centric and radical subgroups with respect to the underlying fusion system, either $\bar{P}$ and $\bar{Q}$ are not $\overline{\mathcal{F}}$-centric or they are not $\overline{\mathcal{F}}$-radical. If $\bar{Q}$ is not $\overline{\mathcal{F}}$-centric, then there is $g \in C_{\bar{S}}(\bar{Q}) \backslash \bar{Q}$ (since $\bar{Q}$ is fully centralized). If $\bar{Q}$ is not $\overline{\mathcal{F}}$-radical, then $O_{p}\left(\operatorname{Aut}_{\overline{\mathcal{F}}}(\bar{Q})\right)>\operatorname{Inn}(\bar{Q})$ and is contained in the Sylow subgroup $\operatorname{Aut}_{\bar{S}}(\bar{Q})(\bar{Q}$ is fully normalized), and thus there is $g \in N_{\bar{S}}(\bar{Q}) \backslash \bar{Q}$ such that $c_{g} \in O_{p}\left(\operatorname{Aut}_{\overline{\mathcal{F}}}(\bar{Q})\right)$. In either case,

$$
g \in Q^{\prime}:=\left\{g \in N_{\bar{S}}(\bar{Q}) \mid c_{g} \in O_{p}\left(\operatorname{Aut}_{\overline{\mathcal{F}}}(\bar{Q})\right)\right\} \quad \text { and } \quad g \notin \bar{Q},
$$

and hence $Q^{\prime}>\bar{Q}$. Also, $Q$ normalizes $Q^{\prime}$ and $Q Q^{\prime}>Q$, so $N_{Q Q^{\prime}}(Q)>Q$, and there is $x \in Q^{\prime} \backslash Q$ such that $x \in N_{S}(Q)$. For any such $x, c_{x} \notin \operatorname{Inn}(Q)$ since $C_{S}(Q) \leq Q$ (recall $P$ is $\mathcal{F}$-centric), and $\left.c_{x}\right|_{\bar{Q}}$ is in $O_{p}(\operatorname{Aut} \overline{\mathcal{F}}(\bar{Q}))$. Also, since $x \in \bar{S} \unlhd S,[x, S] \leq \bar{S}$, and so $c_{x}$ induces the identity on $Q / \bar{Q}$. Thus $c_{x}$ is in the subgroup

$$
\begin{aligned}
U & =\left\{\alpha \in \operatorname{Aut}_{\mathcal{F}}(Q)|\alpha|_{\bar{Q}} \in O_{p}\left(\operatorname{Aut}_{\overline{\mathcal{F}}}(\bar{Q})\right), \alpha \text { induces the identity on } Q / \bar{Q}\right\} \\
& \unlhd \operatorname{Aut}_{\mathcal{F}}(Q) .
\end{aligned}
$$

Since the group of all $\alpha \in \operatorname{Aut}(Q)$ which induce the identity on $\bar{Q}$ and on $Q / \bar{Q}$ is discrete $p$-toral by Lemma 1.13, and is contained in $U$ with $p$-power index, $U$ is a nontrivial normal discrete $p$-toral subgroup of $\operatorname{Aut}_{\mathcal{F}}(Q)$, and $U \leq O_{p}\left(\operatorname{Aut}_{\mathcal{F}}(Q)\right)$. Since $x \in N_{\bar{S}}(Q) \backslash Q, c_{x}$ represents a nontrivial element of $\operatorname{Out}_{S}(Q) \cap O_{p}\left(\operatorname{Out}_{\mathcal{F}}(Q)\right)$, so (v) holds for $P$, and the proof of (5.13) is complete.

We want to show that $\mathcal{K}^{\prime}=\varnothing$. Assume otherwise; then $\overline{\mathcal{K}}^{\prime} \neq \varnothing$ since $P \in \mathcal{K}^{\prime}$ implies $\bar{P} \in \overline{\mathcal{K}}^{\prime}$. Choose $Q$ to be maximal in $\overline{\mathcal{K}}^{\prime}$, and choose $P$ to be maximal among those $P \in \mathcal{K}^{\prime}$ such that $\bar{P}=Q$. Then $P$ is also maximal in $\mathcal{K}^{\prime}$. So by Lemma 3.5(b), $P$ is maximal among subgroups not satisfying the saturation axioms. By Lemma 4.6, this maximality of $P$ implies that (v) does not hold for $P$. Since this contradicts (5.13), we now conclude that $\mathcal{K}^{\prime}=\varnothing$, and hence (by (5.13)) that (v) holds for all $P \in \mathcal{F}^{\bullet}$.

Thus $\mathcal{F}$ is saturated. Also, (v) implies that $\mathcal{H}$ contains all subgroups which are $\mathcal{F}$-centric and $\mathcal{F}$-radical.

Step 6 By [9, Corollary 3.5], $\mathcal{F}^{\bullet}$ contains all subgroups which are $\mathcal{F}$-centric and $\mathcal{F}$-radical, so they are all contained in $\mathcal{H}$ by point (v) in Step 5. Point (a) holds by this together with the definition of $\mathcal{H}=\mathrm{Ob}(\mathcal{T})$. Point (b) holds by the definition 
of $\mathcal{T}$ in Step 2, and (c) holds by the definition of composition (of morphisms between subgroups in $\overline{\mathcal{H}}$ ) in Step 1.

Condition (i) in Definition A.7 (for the inclusion $\overline{\mathcal{L}} \subseteq \mathcal{T}$ ) holds by (a), and since $\bar{S}$ is strongly closed in $\mathcal{F}$ by construction. Condition (ii) (the Frattini condition) holds by the construction in Step 1, and (iii) (invariance of $\overline{\mathcal{L}}$ under $\operatorname{Aut}_{\mathcal{T}}(\bar{S})$-conjugacy) holds by (c). Thus $\overline{\mathcal{L}} \unlhd \mathcal{T}$.

Let $r: \mathcal{T} \rightarrow \mathcal{T}$ be the retraction $r(P)=\bar{P}$ and $r(\varphi)=\bar{\varphi}$ with image $\mathcal{L}_{1}$. There is a natural transformation of functors $r \rightarrow \mathrm{Id}_{\mathcal{T}}$ which sends an object $P$ to the inclusion $\iota_{\bar{P}, P}$. Hence $|r| \simeq \operatorname{Id}_{|\mathcal{T}|}$, and so $\left|\mathcal{L}_{U}\right|$ is a deformation retract of $|\mathcal{T}|$. Also, the inclusion of $|\overline{\mathcal{L}}|$ into $\left|\mathcal{L}_{U}\right|$ is homotopy equivalent to a regular covering with group of deck transformations $G \cong \Gamma / \bar{\Gamma}$ by the last author and Ventura [17, Proposition A.4], and this finishes the proof of (e).

\section{The category $\mathcal{A} u t_{\text {typ }}(\mathcal{L})$}

Fix a $p$-local compact group $(S, \mathcal{F}, \mathcal{L})$. Let $\mathcal{A} u t_{\text {typ }}(\mathcal{L})$ be the groupoid with object set $\operatorname{Aut}_{\text {typ }}^{I}(\mathcal{L})$ and with morphisms the natural transformations. Since a natural transformation $\eta: \alpha \rightarrow \beta$ is determined by $\eta_{S} \in \operatorname{Aut}_{\mathcal{L}}(S)$ ( since $\alpha(S)=\beta(S)=S$ ), morphisms can be described as

$$
\begin{aligned}
\operatorname{Mor}_{\mathcal{A u t} t_{\text {typ }}(\mathcal{L})}(\alpha, \beta) & =\left\{\chi \in \operatorname{Aut}_{\mathcal{L}}(S) \mid \chi \alpha(\varphi)=\beta(\varphi) \chi, \text { all } \varphi \in \operatorname{Mor}(\mathcal{L})\right\} \\
& =\left\{\chi \in \operatorname{Aut}_{\mathcal{L}}(S) \mid \beta=c_{\chi} \circ \alpha\right\}
\end{aligned}
$$

Thus $\chi \in \operatorname{Aut}_{\mathcal{L}}(S)$ corresponds to a natural transformation $\eta$ with $\chi=\eta_{S}$. With this notation, composition of morphisms takes the form

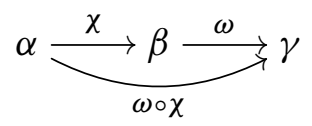

because $\gamma=c_{\omega} \circ \beta=c_{\omega} \circ c_{\chi} \circ \alpha=c_{\omega \circ \chi} \circ \alpha$. Here, $\omega \circ \chi$ is the composite of $\omega$ and $\chi$ in $\operatorname{Aut}_{\mathcal{L}}(S)$.

Composition in $\operatorname{Aut}_{\text {typ }}^{I}(\mathcal{L})$ gives $\mathcal{A} u t_{\text {typ }}(\mathcal{L})$ the structure of a discrete strict monoidal category, where $\alpha \cdot \beta=\alpha \circ \beta$, and where

$$
(\alpha \stackrel{\chi}{\longrightarrow} \beta) \cdot\left(\alpha^{\prime} \stackrel{\chi^{\prime}}{\longrightarrow} \beta^{\prime}\right)=\left(\alpha \circ \alpha^{\prime} \stackrel{\beta\left(\chi^{\prime}\right) \circ \chi}{=\chi \circ \alpha\left(\chi^{\prime}\right)} \beta \circ \beta^{\prime}\right) .
$$

This structure makes the nerve $\mathcal{N} \mathcal{A} u t_{\text {typ }}(\mathcal{L})$ into a simplicial group, and its geometric realization $\left|\mathcal{A} u t_{\text {typ }}(\mathcal{L})\right|$ becomes a topological group. The projection from $\mathcal{A} u t_{\text {typ }}(\mathcal{L})$ 
onto $\operatorname{Out}_{\text {typ }}(\mathcal{L})$ induces a map of simplicial groups

$$
\mathcal{N} \mathcal{A} u t_{\text {typ }}(\mathcal{L}) \stackrel{\text { pr }}{\longrightarrow} \operatorname{Out}_{\text {typ }}(\mathcal{L}),
$$

where now $\operatorname{Out}_{\text {typ }}(\mathcal{L})$, by abuse of language, denotes the discrete simplicial group with vertex set $\operatorname{Out}_{\text {typ }}(\mathcal{L})$. This projection is explicitly given by

$$
\operatorname{pr}\left(\alpha_{0} \longrightarrow \alpha_{1} \longrightarrow \cdots \longrightarrow \alpha_{n}\right)=\left[\alpha_{0}\right] \quad\left(=\left[\alpha_{1}\right]=\cdots=\left[\alpha_{n}\right]\right),
$$

and it sends $\pi_{0}\left(\left|\mathcal{A} u t_{\text {typ }}(\mathcal{L})\right|\right)$ isomorphically onto $\operatorname{Out}_{\text {typ }}(\mathcal{L})$.

The evaluation functor $\mathcal{A} u t_{\text {typ }}(\mathcal{L}) \times \mathcal{L} \rightarrow \mathcal{L}$ induces an action of the simplicial group $\mathcal{N} \mathcal{A} u t_{\text {typ }}(\mathcal{L})$ on the simplicial set $\mathcal{N} \mathcal{L}$ as follows. In dimension $0, \mathcal{N}_{0} \mathcal{A} u t_{\text {typ }}(\mathcal{L})=$ $\operatorname{Aut}_{\text {typ }}^{I}(\mathcal{L})$ acts on $\mathcal{N}_{0} \mathcal{L}=\operatorname{Ob}(\mathcal{L})$ in the obvious way. For $\chi \in \mathcal{N}_{1} \mathcal{A} u t_{\text {typ }}(\mathcal{L})$ and $\varphi \in \operatorname{Mor}_{\mathcal{L}}(P, Q)=\mathcal{N}_{1} \mathcal{L}$,

$$
(\alpha \stackrel{\chi}{\longrightarrow} \beta)(P \stackrel{\varphi}{\longrightarrow} Q)=\left(\alpha(P) \underset{=\chi_{Q} \circ \alpha(\varphi)}{\stackrel{\beta(\varphi) \circ \chi_{P}}{\longrightarrow}} \beta(Q)\right),
$$

and this extends naturally to higher dimensional sequences. The simplicial action induces an action of the topological group $\left|\mathcal{A} u t_{\text {typ }}(\mathcal{L})\right|$ on the space $|\mathcal{L}|$.

Our aim is to describe maps $B G \rightarrow B\left|\mathcal{N} \mathcal{A} u t_{\text {typ }}(\mathcal{L})\right|$ via twisting functions (see, eg, Curtis [11, Definition 3.14]). We need to show extensions of the type constructed in Theorem 5.4 realize certain types of topological fibre bundles, and the relevant obstruction theory is encoded in the simplicial equalities that characterize the twisting functions.

For any (discrete) group $G$, let $\mathcal{E}(G)$ denote the category whose objects are the elements of $G$, with a unique morphism between each pair of objects. Let $\mathcal{B}(G)$ be the category with a single object $\bullet$, and with $G$ as the automorphism group of that object. Then $G$ acts on $\mathcal{E}(G)$ by translation, and the quotient category can be identified with $\mathcal{B}(G)$. The geometric realizations of $\mathcal{E}(G)$ and $\mathcal{B}(G)$ are the universal contractible free $G$-space $E G$, and the classifying space $B G$, respectively.

For a discrete group $G$, we will use the simplicial set $\mathcal{N B}(G)^{\text {op }}$ as a model for $B G$. This allows us to conveniently denote simplices in $\mathcal{N}_{n} \mathcal{B}(G)^{\text {op }}$ by the usual bar notation

$$
\boldsymbol{g}=\left[g_{1}\left|g_{2}\right| \cdots \mid g_{n}\right]:=\left(\bullet \stackrel{g_{1}}{\longleftarrow} \bullet \stackrel{g_{2}}{\longleftarrow} \bullet \cdots \bullet \stackrel{g_{n}}{\longleftarrow} \bullet\right),
$$

where $g_{i} \in G$. We generally omit the superscript "op" from the notation. More generally, for any small category $\mathcal{C}$, we will consider the nerve of the opposite category $\mathcal{C}^{\text {op }}$ as a 
model for the nerve of $\mathcal{C}$. Thus, for example, we consider face maps as

$$
\begin{aligned}
& d_{0}\left(c_{0} \stackrel{f_{1}}{\longleftarrow} c_{1} \stackrel{f_{2}}{\longleftarrow} c_{2} \cdots \stackrel{f_{n}}{\longleftarrow} c_{n}\right)=\left(c_{1} \stackrel{f_{2}}{\longleftarrow} c_{2} \cdots \stackrel{f_{n}}{\longleftarrow} c_{n}\right), \\
& d_{1}\left(c_{0} \stackrel{f_{1}}{\longleftarrow} c_{1} \stackrel{f_{2}}{\longleftarrow} c_{2} \cdots \stackrel{f_{n}}{\longleftarrow} c_{n}\right)=\left(c_{0} \stackrel{f_{1} \circ f_{2}}{\longleftarrow} c_{2} \cdots \stackrel{f_{n}}{\longleftarrow} c_{n}\right),
\end{aligned}
$$

etc.

To each simplicial group $K$, one associates a simplicial set $\bar{W}(K)$ (cf [11, Definition 3.20] or Goerss and Jardine [13, Section V.4]), by setting $\bar{W}_{0}(K)=*$,

$$
\bar{W}_{n}(K)=K_{n-1} \times K_{n-2} \times \cdots \times K_{0} \text { for } n>0,
$$

and with face and degeneracy maps

$$
\begin{aligned}
& d_{i}\left(\kappa_{n-1}, \ldots, \kappa_{0}\right)= \begin{cases}\left(\kappa_{n-2}, \ldots, \kappa_{0}\right) & \text { if } i=0, \\
\left(d_{i-1} \kappa_{n-1}, \ldots,\left(d_{0} \kappa_{n-i}\right) \kappa_{n-i-1}, \ldots, \kappa_{0}\right) & \text { if } 0<i<n, \\
\left(d_{n-1} \kappa_{n-1}, \ldots, d_{1} \kappa_{1}\right) & \text { if } i=n,\end{cases} \\
& s_{i}\left(\kappa_{n-1}, \ldots, \kappa_{0}\right)=\left(s_{i-1} \kappa_{n-1}, \ldots, s_{0} \kappa_{n-i}, 1, \kappa_{n-i-1}, \ldots, \kappa_{0}\right) \quad \text { all } 0 \leq i \leq n \text {, }
\end{aligned}
$$

for $\kappa_{i} \in K_{i}$. Then $|\bar{W}(K)| \simeq B|K|$, and so we can take $\bar{W}\left(\mathcal{N} \mathcal{A} u t_{\text {typ }}(\mathcal{L})\right)$ as a simplicial model for classifying space of the topological group $\left|\mathcal{A} u t_{\text {typ }}(\mathcal{L})\right|$.

A twisting function $t$ from a simplicial set $X$ to a simplicial group $U$ is a collection of maps $t_{n}: X_{n} \rightarrow U_{n-1}$ satisfying certain identities formulated in [11, Definition 3.14]. When $X=\mathcal{N} \mathcal{B}(G)$ and $U=\mathcal{N} \mathcal{A} u t_{\text {typ }}(\mathcal{L})$, a twisting function $\phi: \mathcal{N B}(G) \rightarrow$ $\mathcal{N} \mathcal{A} u t_{\text {typ }}(\mathcal{L})$ is a collection of maps

$$
\phi_{n}: \mathcal{N}_{n} \mathcal{B}(G) \longrightarrow \mathcal{N}_{n-1} \mathcal{A} u t_{\text {typ }}(\mathcal{L}) \quad(\text { all } n \geq 1)
$$

satisfying the relations

$$
\begin{aligned}
\phi_{n-1}\left(d_{i} \boldsymbol{g}\right) & =d_{i-1} \phi_{n}(\boldsymbol{g}) & & \text { for } 2 \leq i \leq n, \\
\phi_{n-1}\left(d_{1} \boldsymbol{g}\right) & =d_{0} \phi_{n}(\boldsymbol{g}) \cdot \phi_{n-1}\left(d_{0} \boldsymbol{g}\right), & & \\
\phi_{n+1}\left(s_{i} \boldsymbol{g}\right) & =s_{i-1} \phi_{n}(\boldsymbol{g}) & & \text { for } i \geq 1, \\
\phi_{n+1}\left(s_{0} \boldsymbol{g}\right) & =\mathbf{1}, & &
\end{aligned}
$$

for all $n \geq 1$ and for all $\boldsymbol{g} \in \mathcal{N}_{n} \mathcal{B}(G)$. Here, $\mathbf{1}$ denotes the identity element in $\mathcal{N}_{n} \mathcal{A} u t_{\text {typ }}(\mathcal{L})$.

To a twisting function $\phi=\left\{\phi_{n}\right\}_{n \geq 1}$ as above, one associates the simplicial map

$$
\varphi: \mathcal{N B}(G) \longrightarrow \bar{W}\left(\mathcal{N} \mathcal{A} u t_{\text {typ }}(\mathcal{L})\right)
$$


where for each $\boldsymbol{g}=\left[g_{1}|\cdots| g_{n}\right] \in \mathcal{N}_{n} \mathcal{B}(G)$,

$$
\begin{aligned}
\varphi(\boldsymbol{g}) & =\left(\phi_{n}(\boldsymbol{g}), \phi_{n-1}\left(d_{0} \boldsymbol{g}\right), \ldots, \phi_{1}\left(d_{0}^{n-1} \boldsymbol{g}\right)\right) \\
& =\left(\phi_{n}\left(\left[g_{1}|\cdots| g_{n}\right]\right), \phi_{n-1}\left(\left[g_{2}|\cdots| g_{n}\right]\right), \ldots, \phi_{1}\left(\left[g_{n}\right]\right)\right) .
\end{aligned}
$$

It is not hard to see that the simplicial equalities (6.5) are designed so that $\varphi$ is a simplicial map. For example, $\varphi$ commutes with the face maps by (6.5) together with the face relations

$$
d_{0}^{j} d_{i}= \begin{cases}d_{i-j} d_{0}^{j} & \text { if } j \leq i, \\ d_{0}^{j+1} & \text { if } j \geq i,\end{cases}
$$

and formal manipulations.

The following lemma will be needed.

Lemma 6.1 Fix a linking triple $(S, \mathcal{F}, \mathcal{L})$, and an extension pair $U=(\widehat{\Gamma}, \tau)$ for $\mathcal{L}$ and $G$. Let $\rho: \widehat{\Gamma} \rightarrow G$ be the surjection with kernel $\Gamma:=\operatorname{Aut}_{\mathcal{L}}(S)$, and let $t_{U}: G \rightarrow \widehat{\Gamma}$ be a regular section of $\rho$ (ie $t_{U}$ is a right inverse for $\rho$ as a map of sets, and $t_{U}(1)=1$ ). Define maps of sets

$$
t: G \rightarrow \operatorname{Aut}_{\mathrm{typ}}^{I}(\mathcal{L}) \text { by setting } t=\tau \circ t_{U},
$$

(6.7) $v: G \times G \rightarrow \operatorname{Inn}(\mathcal{L}) \quad$ such that $t(g) t(h)=v(g, h) t(g h)$ for all $g, h \in G$, $\chi: G \times G \rightarrow \operatorname{Aut}_{\mathcal{L}}(S) \quad$ such that $t_{U}(g) t_{U}(h)=\chi(g, h) t_{U}(g h)$ for all $g, h \in G$.

Then the following hold.

(a) For each $g, h \in G, v(g, h)=c_{\chi(g, h)} \in \operatorname{Inn}(\mathcal{L})$.

(b) For each $g \in G, \chi(1, g)=\chi(g, 1)=1$.

(c) For each $g, h, k \in G, \chi(g, h) \chi(g h, k)=t(g)(\chi(h, k)) \chi(g, h k)$.

Proof Part (a) follows by the commutativity of triangle ( $\dagger$ ) in Definition 5.3(a), and (b) is immediate from the definition of $\chi$. It remains to prove (c).

By definition,

$$
\begin{aligned}
\left(t_{U}(g) t_{U}(h)\right) t_{U}(k) & =\chi(g, h) \chi(g h, k) \cdot t_{U}(g h k), \\
t_{U}(g)\left(t_{U}(h) t_{U}(k)\right) & ={ }^{t_{U}(g)} \chi(h, k) \cdot \chi(g, h k) \cdot t_{U}(g h k) \\
& =t(g)(\chi(h, k)) \cdot \chi(g, h k) \cdot t_{U}(g h k),
\end{aligned}
$$

where the last equality holds by the commutativity of triangle ( $\$$ ) in Definition 5.3(a). The claim now follows by the associativity of multiplication in $\Gamma$. 
We are now ready to show for given $\mathcal{L}$ and $G$, there is a bijective correspondence between twisting functions from $\mathcal{N B}(G)$ to $\mathcal{N} \mathcal{A} u t_{\text {typ }}(\mathcal{L})$ and extension pairs for $\mathcal{L}, G$.

Proposition 6.2 Fix a linking triple $(S, \mathcal{F}, \mathcal{L})$ and a finite group $G$.

(a) Let $U=(\widehat{\Gamma}, \tau)$ be an extension pair for $\mathcal{L}$ and $G$, and let $t_{U}: G \rightarrow \widehat{\Gamma}$ be a regular section. Then there is a unique twisting function $\phi=\phi_{U}: \mathcal{N B}(G) \rightarrow$ $\mathcal{N} \mathcal{A} u t_{\text {typ }}(\mathcal{L})$ such that

(i) for all $g \in G, \tau \circ t_{U}(g)=\phi_{1}([g])$,

(ii) for all $g, h \in G, t_{U}(g) t_{U}(h)=\phi_{2}([g \mid h]) t_{U}(g h)$.

(b) Let $\phi=\left\{\phi_{n}\right\}$ be any twisting function from $\mathcal{N B}(G)$ to $\mathcal{N} \mathcal{A} u t_{\text {typ }}(\mathcal{L})$. There is an extension pair $U=(\widehat{\Gamma}, \tau)$, and a regular section $t_{U}: G \rightarrow \widehat{\Gamma}$, such that $\phi_{U}=\phi$.

Proof (a) Let $U=(\widehat{\Gamma}, \tau)$ be an extension pair for $\mathcal{L}$ and $G$, and fix a regular section $t_{U}: G \rightarrow \widehat{\Gamma}$ of the natural projection. Since a simplex in the nerve of a category is determined by its 1 -faces, there is at most one twisting function $\phi$ which satisfies (i) and (ii). We will prove that such a twisting function exists.

We define $t: G \rightarrow \operatorname{Aut}_{\text {typ }}^{I}(\mathcal{L}), v: G \times G \rightarrow \operatorname{Inn}(\mathcal{L})$ and $\chi: G \times G \rightarrow \operatorname{Aut}_{\mathcal{L}}(S)$, as in (6.7). For $\boldsymbol{g}=\left[g_{1}|\cdots| g_{n}\right]$ and $i \leq j$, write $g_{i, j}=g_{i} g_{i+1} \cdots g_{j}$. Define $\phi$ by setting

$$
\begin{aligned}
& \phi_{n}\left(\left[g_{1}\left|g_{2}\right| \cdots \mid g_{n}\right]\right) \\
&=\left(t\left(g_{1}\right) \stackrel{\chi\left(g_{1}, g_{2}\right)}{\longleftarrow} t\left(g_{1,2}\right) t\left(g_{2}\right)^{-1}=v\left(g_{1}, g_{2}\right)^{-1} t\left(g_{1}\right)\right. \\
& \stackrel{\chi\left(g_{1}, g_{2}\right)^{-1} \chi\left(g_{1}, g_{2,3}\right)}{\longleftarrow} \cdots t t\left(g_{1, n-1}\right) t\left(g_{2, n-1}\right)^{-1}=v\left(g_{1}, g_{2, n-1}\right)^{-1} t\left(g_{1}\right) \\
&\left.\stackrel{\chi\left(g_{1}, g_{2, n-1}\right)^{-1} \chi\left(g_{1}, g_{2, n}\right)}{\longleftarrow} t\left(g_{1, n}\right) t\left(g_{2, n}\right)^{-1}=v\left(g_{1}, g_{2, n}\right)^{-1} t\left(g_{1}\right)\right) .
\end{aligned}
$$

The equalities between the objects hold by (6.7) (the definition of $v$ ).

The first and third relations in (6.5) clearly hold for $\phi$. Also, $\phi_{n}\left(\left[g_{1}|\cdots| g_{n}\right]\right)$ is the identity sequence if $g_{1}=1$, which implies the fourth identity in (6.5). Thus we need only check the second relation, namely that

$$
d_{0} \phi_{n}\left(\left[g_{1}|\cdots| g_{n}\right]\right) \cdot \phi_{n-1}\left(\left[g_{2}|\cdots| g_{n}\right]\right)=\phi_{n-1}\left(\left[g_{1} g_{2}\left|g_{3}\right| \cdots \mid g_{n}\right]\right)
$$

for each $g_{1}, \ldots, g_{n} \in G$. Each side of (6.9) lies in $\mathcal{N}_{n-2} \mathcal{A} u t_{\text {typ }}(\mathcal{L})$, ie a sequence of $(n-1)$ objects and $(n-2)$ morphisms. The objects on each side of (6.9) are the same, since

$$
t\left(g_{1, m}\right) t\left(g_{2, m}\right)^{-1} \circ t\left(g_{2, m}\right) t\left(g_{3, m}\right)^{-1}=t\left(g_{1, m}\right) t\left(g_{3, m}\right)^{-1}
$$


for all $m \geq 2$ (where we now set $g_{3,2}=1$ and $g_{2,2}=g_{2}$ ). To show that the morphisms in the sequences coincide, we must show that

$$
\begin{aligned}
\left(\chi\left(g_{1}, g_{2, m}\right)^{-1} \chi\left(g_{1}, g_{2, m+1}\right)\right) \cdot\left(\chi\left(g_{2}, g_{3, m}\right)^{-1} \chi\left(g_{2}, g_{3, m+1}\right)\right) & \\
= & \chi\left(g_{1,2}, g_{3, m}\right) \chi\left(g_{1,2}, g_{3, m+1}\right)
\end{aligned}
$$

for all $m \geq 2$ (where again $g_{3,2}=1$ ). By (6.2), the left-hand side of this equation takes the form

$$
\begin{aligned}
& \left(\chi\left(g_{1}, g_{2, m}\right)^{-1} \circ \chi\left(g_{1}, g_{2, m+1}\right)\right) \circ\left(t\left(g_{1, m+1}\right) t\left(g_{2, m+1}^{-1}\right)\right)\left(\chi\left(g_{2}, g_{3, m}\right)^{-1}\right. \\
& \left.\quad \circ \chi\left(g_{2}, g_{3, m+1}\right)\right) \\
& =\chi\left(g_{1}, g_{2, m}\right)^{-1} \chi\left(g_{1}, g_{2, m+1}\right) \\
& \quad \circ c_{\chi\left(g_{1}, g_{2, m+1}\right)}^{-1}\left(g_{1}\right)\left(\chi\left(g_{2}, g_{3, m}\right)^{-1} \chi\left(g_{2}, g_{3, m+1}\right)\right) \\
& =\chi\left(g_{1}, g_{2, m}\right)^{-1} t\left(g_{1}\right)\left(\chi\left(g_{2}, g_{3, m}\right)\right)^{-1} \\
& \quad \circ t\left(g_{1}\right)\left(\chi\left(g_{2}, g_{3, m+1}\right)\right) \chi\left(g_{1}, g_{2, m+1}\right) \\
& =\left(\chi\left(g_{1}, g_{2}\right) \chi\left(g_{1} g_{2}, g_{3, m}\right)\right)^{-1} \chi\left(g_{1}, g_{2}\right) \chi\left(g_{1,2}, g_{3, m+1}\right) \\
& =\chi\left(g_{1,2}, g_{3, m}\right)^{-1} \chi\left(g_{1,2}, g_{3, m+1}\right) .
\end{aligned}
$$

Here, the first equality in (6.10) follows from (6.7) and the definition of $v$, and the third follows upon applying Lemma 6.1(c) twice. This finishes the proof that $\phi$ is a twisting function.

By construction, $\phi_{1}([g])=t(g)=\tau \circ t_{U}(g)$, and so $\phi$ satisfies (i). Upon setting $n=2$ in (6.8), we may identify $\phi_{2}([g \mid h])$ with $\chi(g, h)$, and so (ii) holds by Lemma 6.1.

(b) Fix a twisting function $\phi$, and define $t: G \rightarrow \operatorname{Aut}_{\text {typ }}^{I}(\mathcal{L})$ by $t(g):=\phi_{1}([g])$. Then $t(1)=1$.

For each $g, h \in G$, the formulas in (6.5) for faces of $\phi_{2}([g \mid h])$ take the form

$$
d_{1} \phi_{2}([g \mid h])=t(g) \quad \text { and } \quad d_{0} \phi_{2}([g \mid h])=t(g h) \cdot t(h)^{-1}
$$

So there is $\chi(g, h) \in \mathcal{N}_{1} \mathcal{A} u t_{\text {typ }}(\mathcal{L})=\operatorname{Aut}_{\mathcal{L}}(S)$ such that

$$
\phi_{2}([g \mid h])=\left(t(g) \stackrel{\chi(g, h)}{\longleftarrow} t(g h) t(h)^{-1}\right) .
$$

By $(6.1), t(g)=c_{\chi(g, h)} \circ t(g h) t(h)^{-1}$, and hence

$$
t(g) t(h)=c_{\chi(g, h)} \cdot t(g h) .
$$

Also, by the degeneracy relations in (6.5), for each $g \in G, \chi(g, 1)=1=\chi(1, g)$. 
Similarly, for each $g, h, k \in G, \phi_{3}([g|h| k])$ has faces

$$
\begin{aligned}
& d_{2} \phi_{3}([g|h| k])=\phi_{2}([g \mid h]), \\
& d_{1} \phi_{3}([g|h| k])=\phi_{2}([g \mid h k]), \\
& d_{0} \phi_{3}([g|h| k])=\phi_{2}([g h \mid k]) \cdot \phi_{2}([h \mid k])^{-1}
\end{aligned}
$$

by (6.5). Hence $\phi_{3}([g|h| k])$ takes the form

$$
\phi_{3}([g|h| k])=\left(t(g) \stackrel{\chi(g, h)}{\longleftarrow} t(g h) t(h)^{-1} \stackrel{\chi_{1}}{\longleftarrow} t(g h k) t(h k)^{-1}\right)
$$

for some $\chi_{1} \in \operatorname{Aut}_{\mathcal{L}}(S)$. The second formula in (6.12) implies $\chi(g, h) \circ \chi_{1}=\chi(g, h k)$, and hence $\chi_{1}=\chi(g, h)^{-1} \chi(g, h k)$. The third formula in (6.12) now implies

$$
\begin{aligned}
\left(t(g h) t(h)^{-1} \stackrel{\chi(g, h)^{-1} \chi(g, h k)}{\longleftarrow} t(g h k) t(h k)^{-1}\right) \cdot( & \left.t(h) \stackrel{\chi(h, k)}{\longleftarrow} t(h k) t(k)^{-1}\right) \\
& =\left(t(g h) \stackrel{\chi(g h, k)}{\longleftarrow} t(g h k) t(k)^{-1}\right) .
\end{aligned}
$$

Hence by definition of the monoidal structure on $\mathcal{A} u t_{\text {typ }}(\mathcal{L})$ (see (6.2)),

$$
\chi(g h, k)=\chi(g, h)^{-1} \chi(g, h k) \cdot\left(t(g h k) t(h k)^{-1}\right)(\chi(h, k)) .
$$

By $(6.11), c_{\chi(g, h k)} \circ t(g h k) t(h k)^{-1}=t(g)$. Hence

$$
\begin{aligned}
\chi(g, h) \chi(g h, k) & =\chi(g, h k) \cdot\left(c_{\chi(g, h k)}^{-1} \circ t(g)\right)(\chi(h, k)) \\
& =t(g)(\chi(h, k)) \chi(g, h k) .
\end{aligned}
$$

Now define $\hat{\Gamma}=\operatorname{Aut}_{\mathcal{L}}(S) \times G$, with group multiplication

$$
(a, g) \cdot(b, h)=(a \cdot t(g)(b) \cdot \chi(g, h), g h)
$$

for each $g, h \in G$ and each $a, b \in \operatorname{Aut}_{\mathcal{L}}(S)$. Define $\tau: \widehat{\Gamma} \rightarrow \operatorname{Aut}_{\text {typ }}^{I}(\mathcal{L})$ by setting $\tau(a, g)=c_{a} \circ t(g)$. For each $g, h, k \in G$ and each $a, b, c \in \operatorname{Aut}_{\mathcal{L}}(S)$,

$$
\begin{aligned}
((a, g) \cdot(b, h)) \cdot(c, k) & =(a \cdot t(g)(b) \cdot \chi(g, h) \cdot t(g h)(c) \cdot \chi(g h, k), g h k) \\
& =(a \cdot t(g)(b) \cdot t(g) t(h)(c) \cdot \chi(g, h) \cdot \chi(g h, k), g h k) \\
& =(a \cdot t(g)(b) \cdot t(g) t(h)(c) \cdot t(g)(\chi(h, k)) \cdot \chi(g, h k), g h k) \\
& =(a, g) \cdot(b \cdot t(h)(c) \cdot \chi(h, k), h k) \\
& =(a, g) \cdot((b, h) \cdot(c, k)),
\end{aligned}
$$

where the second and third equalities follow from (6.11) and (6.13) respectively. Thus multiplication in $\widehat{\Gamma}$ is associative, and $\widehat{\Gamma}$ is a group, with the obvious identity and 
inverses. Similarly,

$$
\begin{aligned}
\tau((a, g) \cdot(b, h)) & =\tau(a \cdot t(g)(b) \cdot \chi(g, h), g h)=c_{a} \circ c_{t(g)(b)} \circ c_{\chi(g, h)} \circ t(g h) \\
& =c_{a} \circ t(g) c_{b} t(g)^{-1} \circ c_{\chi(g, h)} t(g h)=c_{a} \circ t(g) c_{b} t(g)^{-1} \circ t(g) t(h) \\
& =\tau(a, g) \tau(b, h),
\end{aligned}
$$

and so $\tau$ is a homomorphism.

The triangle ( $\dagger$ ) in Definition 5.3)(a) commutes by definition of $\tau$. Triangle ( $\$$ ) commutes since

$$
(a, g)(b, 1)(a, g)^{-1}=\left(a t(g)(b) a^{-1}, 1\right)=(\tau(a, g)(b), 1) \in \Gamma
$$

for each $(a, g) \in \hat{\Gamma}$ and each $b \in \operatorname{Aut}_{\mathcal{L}}(S)$. This shows that $U:=(\widehat{\Gamma}, \tau)$ is an extension pair for $\mathcal{L}$ and $G$.

Finally, for $g \in G$, set $t_{U}(g)=(1, g) \in \hat{\Gamma}$. Then $t_{U}$ is a regular section of the obvious projection $\rho: \widehat{\Gamma} \rightarrow G$. By the definitions, $\tau\left(t_{U}(g)\right)=t(g)=\phi_{1}([g])$, while for $g, h \in G$,

$$
t_{U}(g) t_{U}(h)=(1, g) \cdot(1, h)=(\chi(g, h), g h)=(\chi(g, h), 1) \cdot(1, g h) .
$$

Upon identifying $(\chi(g, h), 1) \in \widehat{\Gamma}$ with $\phi_{2}([g \mid h])$, we obtain (i) and (ii).

A twisting function $\phi: \mathcal{N B}(G) \rightarrow \mathcal{N} \mathcal{A} u t_{\text {typ }}(\mathcal{L})$ determines a map

$$
B G \longrightarrow B\left|\mathcal{N} \mathcal{A} u t_{\text {typ }}(\mathcal{L})\right|
$$

(see (6.6)), and hence determines a fibre bundle over $B G$ with fibre $|\mathcal{L}|$ and structure group $\left|\mathcal{A} u t_{\text {typ }}(\mathcal{L})\right|$. This pullback bundle is the realization of a simplicial set $E(\phi)=$ $|\mathcal{L}| \times_{\phi} B G$ : a twisted cartesian product which is described as follows (cf $[11,(6.4)]$ ).

Definition 6.3 For any twisting function $\phi: \mathcal{N B}(G) \rightarrow \mathcal{N} \mathcal{A} u t_{\text {typ }}(\mathcal{L})$, let $E(\phi)$ be the simplicial set with $n$-simplices $E(\phi)_{n}=\mathcal{N}_{n} \mathcal{L} \times \mathcal{N}_{n} \mathcal{B}(G)$, and with face and degeneracy maps

$$
\begin{aligned}
& d_{i}(\boldsymbol{\xi}, \boldsymbol{g})= \begin{cases}\left(\phi_{n}(\boldsymbol{g})^{-1} \cdot d_{0} \xi, d_{0} \boldsymbol{g}\right) & \text { if } i=0, \\
\left(d_{i} \xi, d_{i} \boldsymbol{g}\right) & \text { if } i>0,\end{cases} \\
& s_{i}(\boldsymbol{\xi}, \boldsymbol{g})=\left(s_{i} \boldsymbol{\xi}, s_{i} \boldsymbol{g}\right) \quad \text { for all } i,
\end{aligned}
$$

for all $\xi \in \mathcal{N}_{n} \mathcal{L}$ and all $\boldsymbol{g} \in \mathcal{N}_{n} \mathcal{B}(G)$. Let $p_{\phi}: E(\phi) \rightarrow \mathcal{N B}(G)$ be the natural projection which maps a pair $(\boldsymbol{\xi}, \boldsymbol{g}) \in E(\phi)$ to $\boldsymbol{g}$. 
By [11, Section 6.4], in the situation of Definition $6.3, p_{\phi}$ induces a fibre bundle

$$
\left|p_{\phi}\right|:|E(\phi)| \longrightarrow B G
$$

with fibre $|\mathcal{L}|$ and structure group $\left|\mathcal{A} u t_{\text {typ }}(\mathcal{L})\right|$. We want to identify the nerve of the extension $\mathcal{L}_{U}$ of Definition 5.3 with $E(\phi)$ for the associated twisting function $\phi$. Before we can do this, one more technical lemma is required.

Lemma 6.4 Let $X$ be a simplicial set such that for each $n \geq 2$, the map

$$
D_{n}=: X_{n} \stackrel{\left(\left(d_{2}\right)^{n-1}, d_{0}\right)}{\longrightarrow}\left\{\left(x_{1}, x_{2}\right) \in X_{1} \times X_{n-1} \mid d_{0} x_{1}=\left(d_{1}\right)^{n-1}\left(x_{2}\right) \in X_{0}\right\}
$$

is a bijection. Then there is a category $\mathcal{X}$ with $\operatorname{Ob}(\mathcal{X})=X_{0}$ and $\operatorname{Mor}(\mathcal{X})=X_{1}$, where $f \in X_{1}$ is a morphism from $d_{0} f$ to $d_{1} f$ and $\operatorname{Id}_{x}=s_{0} x$ for $x \in X_{0}$, in which composition is defined as follows. If $f_{1}, f_{2} \in X_{1}$ are composable morphisms (ie if $d_{0} f_{1}=d_{1} f_{2}$ ), then their composite is defined by setting $f_{1} \circ f_{2}=d_{1} x$, where $x \in X_{2}$ is the unique element such that $D_{2}(x)=\left(f_{1}, f_{2}\right)$. In other words, $f_{1} \circ f_{2}=$ $d_{1} \circ D_{2}^{-1}\left(f_{1}, f_{2}\right)$. Furthermore, $\mathcal{N} \mathcal{X} \cong X$ as simplicial sets.

Proof For each $0 \leq i<n$, let $e_{i}^{n}: X_{n} \rightarrow X_{1}$ be the "edge map" induced by the morphism $[1] \rightarrow[n]$ in $\Delta$ with image $\{i, i+1\}$. Thus, for example, $e_{0}^{2}=d_{2}, e_{1}^{2}=d_{0}$, $e_{0}^{n}=\left(d_{2}\right)^{n-1}$ for $n>2$, etc.

For each $n \geq 2$, set

$$
\mathcal{N}_{n} \mathcal{X}=\left\{\left(f_{0}, \ldots, f_{n-1}\right) \in\left(X_{1}\right)^{n} \mid d_{0} f_{i}=d_{1} f_{i+1} \text { for all } 0 \leq i \leq n-2\right\}:
$$

the set of $n$-tuples of composable morphisms. Set

$$
E_{n}=\left(e_{0}^{n}, e_{1}^{n}, \ldots, e_{n-1}^{n}\right): X_{n} \longrightarrow \mathcal{N}_{n} \mathcal{X} \subseteq\left(X_{1}\right)^{n} .
$$

It is easy to check that $E_{n}=\left(\operatorname{Id} \times D_{2}\right) \circ\left(\operatorname{Id} \times D_{3}\right) \circ \cdots \circ D_{n}$, and so $E_{n}$ is a bijection since the $D_{i}$ are. In particular, for each $\left(f_{1}, f_{2}, f_{3}\right) \in \mathcal{N}_{3} \mathcal{X}$, there is a unique $y \in X_{3}$ such that $E_{3}(y)=\left(f_{1}, f_{2}, f_{3}\right)=\left(d_{2}^{2} y, d_{2} d_{0} y, d_{0}^{2} y\right)=\left(d_{2} d_{3} y, d_{0} d_{3} y, d_{0} d_{1} y\right)$ and that

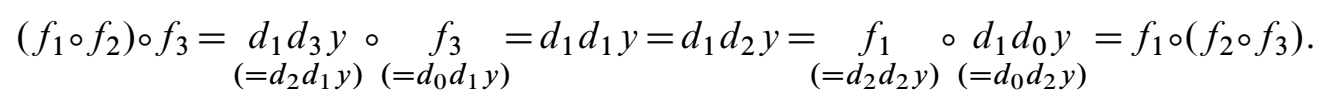

The first equality follows since $D_{2}\left(d_{3} y\right)=\left(d_{2} d_{3} y, d_{0} d_{3} y\right)=\left(f_{1}, f_{2}\right)$, and the fifth since $D_{2}\left(d_{0} y\right)=\left(d_{0}^{2} y, d_{0} d_{0} y\right)=\left(f_{2}, f_{3}\right)$, the second and fourth equalities hold by definition of $D_{2}$, and the third is a simplicial identity. This shows that composition in $\mathcal{X}$ is associative, and hence that $\mathcal{X}$ is a category.

Set $E_{0}=\operatorname{Id}_{X_{0}}, E_{1}=\operatorname{Id}_{X_{1}}$ and $E=\left\{E_{n}\right\}: X \rightarrow \mathcal{N} \mathcal{X}$. By construction, $E$ commutes with face maps on $X_{n}$ for $n \leq 2$. We claim that $d_{i} \circ E_{n}=E_{n-1} \circ d_{i}$ for $n \geq 3$ and 
$0 \leq i \leq n$. This is clear from the definition of $E_{n}$, when $i=0$ or $i=n$, since in these two cases $d_{0}$ and $d_{n}$ on $\mathcal{N}_{n} \mathcal{X}$ are restrictions of the obvious projections $p_{0}, p_{n}:\left(X_{1}\right)^{n} \rightarrow\left(X_{1}\right)^{n-1}$. So assume $0<i<n$, and consider the map

$$
T: X_{n} \stackrel{\left(e_{0}^{n}, \ldots, e_{i-2}^{n}, t, e_{i+1}^{n}, \ldots, e_{n-1}^{n}\right)}{\longrightarrow}\left(X_{1}\right)^{i-1} \times X_{2} \times\left(X_{1}\right)^{n-i-1},
$$

where $t$ is induced by the morphism [2] $\rightarrow[n]$ with image $\{i-1, i, i+1\}$. Then $E_{n}=\left(\mathrm{Id},\left(d_{2}, d_{0}\right), \mathrm{Id}\right) \circ T$, while $E_{n-1} \circ d_{i}=\left(\mathrm{Id}, d_{1}, \mathrm{Id}\right) \circ T$. In other words, for each $x \in X_{n}$ with $E_{n}(x)=\left(f_{1}, \ldots, f_{n}\right)$, we have $E_{n-1}\left(d_{i} x\right)=\left(f_{1}, \ldots, f_{i-1}, f_{i}\right.$ 。 $\left.f_{i+1}, \ldots, f_{n}\right)=d_{i}\left(E_{n}(x)\right)$.

This proves that $E$ commutes with face maps, and it is easily seen (by the choice of identity morphisms) to commute with degeneracies. Since all of the $E_{i}$ are bijections, $E$ is an isomorphism of simplicial sets.

For any extension pair $U=(\hat{\Gamma}, \tau)$ for $\mathcal{L}$ and $G$, we let pr: $\mathcal{L}_{U} \rightarrow \mathcal{B}(G)$ be the functor defined by setting $\operatorname{pr}(\llbracket \varphi, \gamma \rrbracket)=\rho(\gamma)$.

Proposition 6.5 Fix a linking triple $(S, \mathcal{F}, \mathcal{L})$, a finite group $G$, and an extension pair $U=(\widehat{\Gamma}, \tau)$ for $\mathcal{L}$ and $G$ with a regular section $t_{U}: G \rightarrow \widehat{\Gamma}$. Let $\phi=\phi_{U}$ be the associated twisting function, as in Proposition 6.2(a). Then $\mathcal{N} \mathcal{L}_{U} \cong E(\phi)$, via a simplicial isomorphism which commutes with the projections to $\mathcal{N B}(G)$. In particular,

$$
\left|\mathcal{L}_{U}\right| \stackrel{\left|\mathrm{pr}_{U}\right|}{\longrightarrow} B G
$$

is a fibre bundle over $B G$ with fibre $|\mathcal{L}|$ and structure group $\left|\mathcal{A} u t_{\text {typ }}(\mathcal{L})\right|$.

Proof We first claim, for each $n \geq 2$, that the map

$D_{n}=\left(d_{2}^{n-1}, d_{0}\right): E(\phi)_{n}$

$$
\longrightarrow\left\{\left(\eta_{1}, \eta_{2}\right) \in E(\phi)_{1} \times E(\phi)_{n-1} \mid d_{0} \eta_{1}=d_{1}^{n-1} \eta_{2} \in E(\phi)_{0}\right\}
$$

is a bijection. For $\boldsymbol{\xi}=\left(P_{0} \stackrel{\varphi_{1}}{\longleftarrow} P_{1} \leftarrow \cdots \leftarrow P_{n}\right)$ and $\boldsymbol{g}=\left[g_{1}|\cdots| g_{n}\right]$,

$$
\begin{aligned}
D_{n}(\boldsymbol{\xi}, \boldsymbol{g})=\left(\left(P_{0} \stackrel{\varphi_{1}}{\longleftarrow} P_{1},\left[g_{1}\right]\right),\left(\phi_{n}(\boldsymbol{g})^{-1} \cdot d_{0} \boldsymbol{\xi},\left[g_{2}|\cdots| g_{n}\right]\right)\right), \\
d_{1}^{n-1}\left(\phi_{n}(\boldsymbol{g})^{-1} \cdot d_{0} \xi,\left[g_{2}|\cdots| g_{n}\right]\right)=\left(\phi_{1}\left(d_{2}^{n-1} \boldsymbol{g}\right)^{-1} \cdot d_{1}^{n-1} d_{0} \xi, *\right) \\
=\left(\phi_{1}\left(\left[g_{1}\right]\right)^{-1} P_{1}, *\right)=d_{0}\left(P_{0} \stackrel{\varphi_{1}}{\longleftarrow} P_{1},\left[g_{1}\right]\right) .
\end{aligned}
$$

For fixed $\boldsymbol{g}, D_{n}$ restricts to a function from $\mathcal{N}_{n} \mathcal{L}$ to the set of pairs $\left(\xi_{1}, \xi_{2}\right) \in$ $\mathcal{N}_{1} \mathcal{L} \times \mathcal{N}_{n-1} \mathcal{L}$ such that

$$
\phi_{1}\left(\left[g_{1}\right]\right)^{-1}\left(d_{0} \xi_{1}\right)=d_{1}^{n-1} \xi_{2}
$$


This function is in fact a bijection, since by (6.14), the last term of $\boldsymbol{\xi}_{2}$ is the first term of $\phi_{1}\left(\left[g_{1}\right]\right)^{-1}\left(d_{0} \xi_{1}\right)$, and since $\phi_{1}\left(\left[g_{1}\right]\right)$ is invertible. Since $\boldsymbol{g}=\left[g_{1}|\cdots| g_{n}\right]$ is determined by $\left[g_{1}\right]$ and $d_{0} g=\left[g_{2}|\cdots| g_{n}\right]$, it follows that $D_{n}$ is a bijection.

By Lemma 6.4, $E(\phi) \cong \mathcal{N} \mathcal{E}(\phi)$, where $\mathcal{E}(\phi)$ is the category with

$$
\operatorname{Ob}(\mathcal{E}(\phi))=E(\phi)_{0}=\operatorname{Ob}(\mathcal{L}) \times\{*\} \quad \text { and } \quad \operatorname{Mor}(\mathcal{E}(\phi))=E(\phi)_{1}=\operatorname{Mor}(\mathcal{L}) \times G,
$$

with source and target defined by $d_{0}$ and $d_{1}$, respectively, with $\operatorname{Id}_{(P, *)}=s_{0}(P, *)=$ $\left(\operatorname{Id}_{P}, 1\right)$, and with composition defined by $d_{1} \circ D_{2}^{-1}$. Let $t$ and $\chi$ be the functions associated to $U$ and $t_{U}$ via (6.7) (we do not use $v$ here). For each $\varphi \in \operatorname{Mor}_{\mathcal{L}}(P, Q)$ and $g \in G$,

$$
\begin{aligned}
& d_{0}(Q \stackrel{\varphi}{\leftarrow} P,[g])=\left(\phi_{1}([g])^{-1}(P), *\right)=\left(t(g)^{-1}(P), *\right), \\
& d_{1}(Q \stackrel{\varphi}{\leftarrow} P,[g])=(Q, *),
\end{aligned}
$$

so $(\varphi, g) \in \operatorname{Mor}_{\mathcal{E}(\phi)}\left(\left(t(g)^{-1}(P), *\right),(Q, *)\right)$.

We next describe composition in $\mathcal{E}(\phi)$. For each

$$
\eta=(R \stackrel{\varphi}{\leftarrow} Q \stackrel{\lambda}{\leftarrow} P,[g \mid h]) \in E(\phi)_{2},
$$

$d_{1} \eta=(\varphi \circ \lambda,[g h])$ and $d_{2} \eta=(\varphi,[g])$, while

$$
\begin{aligned}
d_{0} \eta & =\left(\phi_{2}([g \mid h])^{-1}(Q \stackrel{\lambda}{\leftarrow} P),[g]\right) \\
& =\left(\left(t(g) \stackrel{\chi(g, h)}{\longleftarrow} t(g h) t(h)^{-1}\right)^{-1} \cdot(Q \stackrel{\lambda}{\leftarrow} P),[g]\right) \\
& =\left(t(g)^{-1}(Q) \stackrel{\psi}{\leftarrow} t(h) t(g h)^{-1}(P),[g]\right)
\end{aligned}
$$

where by formula (6.3) for the action of $\operatorname{Mor}\left(\mathcal{A} u t_{\text {typ }}(\mathcal{L})\right)$ on $\operatorname{Mor}(\mathcal{L})$,

$$
\lambda=\left(t(g h) t(h)^{-1} \stackrel{\chi(g, h)}{\longrightarrow} t(g)\right) \cdot \psi=t(g)(\psi) \circ \chi(g, h) .
$$

In other words, composition in $\mathcal{E}(\phi)$ satisfies

$$
(\varphi, g) \circ(\psi, h)=(\varphi \circ \lambda, g h)=(\varphi \circ t(g)(\psi) \circ \chi(g, h), g h)
$$

It remains to show that $\mathcal{E}(\phi) \cong \mathcal{L}_{U}$. By construction, each morphism in $\mathcal{L}_{U}$ has the form $\llbracket \varphi, t_{U}(g) \rrbracket$ for some unique $\varphi$ and $g$. Define $\omega: \mathcal{L}_{U} \rightarrow \mathcal{E}(\phi)$ by setting $\omega(P)=(P, *)$ and $\omega\left(\llbracket \varphi, t_{U}(g) \rrbracket\right)=(\varphi, g)$. By $(6.15)$, this preserves composition, and hence is an isomorphism of categories. 


\section{The proof of Theorem A}

Before proving Theorem A, we need one more result, which allows us to compare fibrations with fibre $|\mathcal{L}|_{p}^{\wedge}$ and fibre bundles with fibre $|\mathcal{L}|$.

Proposition 7.1 For each $p$-local compact group $(S, \mathcal{F}, \mathcal{L})$ and each finite group $G$, there is a bijection $\Phi$ from the set of equivalence classes of fibre bundles over $B G$ with fibre $|\mathcal{L}|$ and structure group $\left|\mathcal{A} u t_{\text {typ }}(\mathcal{L})\right|$ to the set of equivalence classes of fibrations over $B G$ with fibre homotopy equivalent to $|\mathcal{L}|_{p}^{\wedge}$ : a bijection which sends the class of a fibre bundle to the equivalence class of its fibrewise $p$-completion.

Proof For any space $X$, let $\operatorname{Aut}(X)$ denote the space of its self homotopy equivalences. Let

$$
\Omega:\left|\mathcal{A} u t_{\text {typ }}(\mathcal{L})\right| \longrightarrow \operatorname{Aut}(|\mathcal{L}|) \quad \text { and } \quad \hat{\Omega}:\left|\mathcal{A} u t_{\text {typ }}(\mathcal{L})\right| \longrightarrow \operatorname{Aut}\left(|\mathcal{L}|_{p}^{\wedge}\right)
$$

be the homomorphisms induced by the evaluation functor $\mathcal{A} u t_{\text {typ }}(\mathcal{L}) \times \mathcal{L} \rightarrow \mathcal{L}$ and by $p$-completion.

By Barratt, Gugenheim and Moore [3, Theorem 5.6], equivalence classes of fibrations over $B G$ with fibre $|\mathcal{L}|_{p}^{\wedge}$ are in one-to-one correspondence with the set of homotopy classes of pointed maps $\left[B G, B \operatorname{Aut}\left(|\mathcal{L}|_{p}^{\wedge}\right)\right]_{*}$. Also, equivalence classes of $\left|\mathcal{A} u t_{\text {typ }}(\mathcal{L})\right|-$ bundles over $B G$ with fibre $|\mathcal{L}|$ are in one-to-one correspondence with the set of homotopy classes of pointed maps $\left[B G, B\left|\mathcal{A} u t_{\text {typ }}(\mathcal{L})\right|\right]_{*}$.

If a map

$$
f: B G \rightarrow B\left|\mathcal{A} u t_{\text {typ }}(\mathcal{L})\right|
$$

classifies an $\left|\mathcal{A} u t_{\text {typ }}(\mathcal{L})\right|$-bundle $\xi_{f}$ with fibre $|\mathcal{L}|$, then $B \widehat{\Omega} \circ f$ classifies the fibrewise $p$-completion $\hat{\xi}_{f}$ of $\xi_{f}$. So we must show that the map

$$
\Phi:\left[B G, B\left|\mathcal{A} u t_{\mathrm{typ}}(\mathcal{L})\right|\right]_{*} \stackrel{B \hat{\Omega}_{\circ}-}{\longrightarrow}\left[B G, B \operatorname{Aut}\left(|\mathcal{L}|_{p}^{\wedge}\right)\right]_{*}
$$

is a bijection.

By [9, Theorem 7.1], $\widehat{\Omega}$ induces a homotopy equivalence after (componentwise) $p-$ completion. Hence $B \operatorname{Aut}\left(|\mathcal{L}|_{p}^{\wedge}\right)$ is the fibrewise $p$-completion of $B \mathcal{A} u t_{\text {typ }}(\mathcal{L})$ over $B \operatorname{Out}\left(|\mathcal{L}|_{p}^{\wedge}\right) \cong B \operatorname{Out}_{\text {typ }}(\mathcal{L})$, and there is a map of fibration sequences

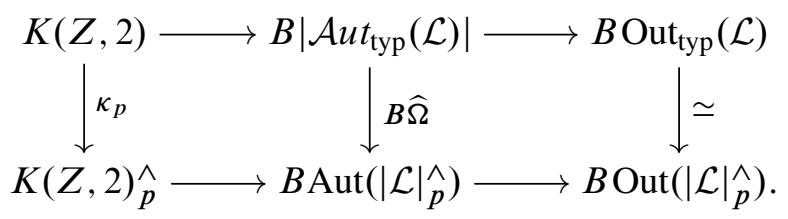


Here, $Z=$ Aut $_{\mathcal{A u t} t_{\text {typ }}(\mathcal{L})}(\mathrm{Id})$, and by (6.1) in Section 6, it can be identified as a subgroup of $Z(S)$. (In fact, $Z=Z(\mathcal{F})$ : the center of the fusion system $\mathcal{F}$.) Hence it is an abelian discrete $p$-toral group of the form $Z \cong\left(\mathbb{Z} / p^{\infty}\right)^{r} \times A$ for some finite abelian $p$-group $A$.

By [4, Proposition VI.5.1], $K\left(\mathbb{Z} / p^{\infty}, 2\right)_{p}^{\wedge} \simeq K\left(\mathbb{Z}_{p}, 3\right), K\left(\mathbb{Q}_{p}, 2\right)_{p}^{\wedge} \simeq *$ and $K\left(\mathbb{Z}_{p}, 2\right)$ is $p$-complete. Hence by the fibre completion lemma [4, Lemma II.4.8], there is a map of fibration sequences

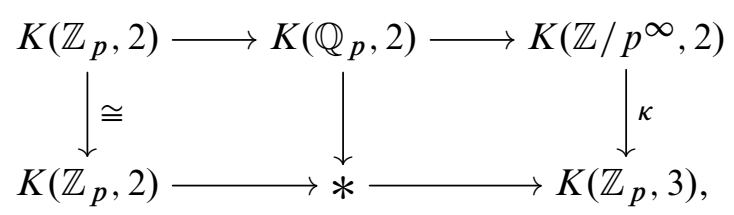

and so hofibre $(\kappa) \simeq K\left(\mathbb{Q}_{p}, 2\right)$. Thus $K(Z, 2)_{p}^{\wedge} \simeq K\left(\left(\mathbb{Z}_{p}\right)^{r}, 3\right) \times K(A, 2)$, and the homotopy fibre of $B \hat{\Omega}$ is equivalent to hofibre $\left(\kappa_{p}\right) \simeq K\left(\left(\mathbb{Q}_{p}\right)^{r}, 2\right)$.

Since $G$ is finite, $H^{i}\left(G ;\left(\mathbb{Q}_{p}\right)^{r}\right)=0$ for all $i \geq 1$. Thus $\Phi$ is a bijection by obstruction theory.

Remark 7.2 In particular, we have shown that each class of fibrations over $B G$ with fibre $|\mathcal{L}|_{p}^{\wedge}$ has a representative which is actually an $\left|\mathcal{A} u t_{\text {typ }}(\mathcal{L})\right|$-bundle. In other words, the structure group of a fibration can always be reduced to $\left|\mathcal{A} u t_{\mathrm{typ}}(\mathcal{L})\right|$.

We are now ready to prove Theorem A, in the following slightly more precise form.

Theorem 7.3 Assume $f: X \rightarrow Y$ is a finite regular covering space with group of deck transformations $G$, where $X \simeq|\overline{\mathcal{L}}|_{p}^{\wedge}$ is the classifying space of a $p$-local compact group $(\bar{S}, \overline{\mathcal{F}}, \overline{\mathcal{L}})$. Then $Y_{p}^{\wedge}$ is the classifying space of a $p$-local compact group $(S, \mathcal{F}, \mathcal{L})$. Furthermore, there is a transporter system $\mathcal{T}$ associated to $\mathcal{F}$ and $\mathcal{L}$ such that $\overline{\mathcal{L}} \unlhd \mathcal{T}$, and such that $\operatorname{Aut}_{\mathcal{T}}(\bar{S}) / \operatorname{Aut}_{\overline{\mathcal{L}}}(\bar{S}) \cong G$.

Proof By Proposition 7.1, there is a fibre bundle $|\overline{\mathcal{L}}| \rightarrow E \rightarrow B G$ with structure group $\left|\mathcal{A} u t_{\text {typ }}(\overline{\mathcal{L}})\right|$ whose fibrewise $p$-completion is equivalent up to homotopy to the fibration $X \rightarrow X \times{ }_{G} E G \rightarrow B G$. This bundle is classified by a map $\varphi$ from $B G$ to $B\left|\mathcal{A} u t_{\text {typ }}(\overline{\mathcal{L}})\right|=\left|\bar{W} \mathcal{A} u t_{\text {typ }}(\overline{\mathcal{L}})\right|$. Since $\bar{W} \mathcal{A} u t_{\text {typ }}(\overline{\mathcal{L}})$ is fibrant (cf [13, Corollary V.6.8]), we can assume that $\varphi$ is the realization of a simplicial map, and hence is determined by a twisting function $\phi$.

By Proposition 6.2(b), $\phi=\phi_{U}$ for some extension pair $U=(\widehat{\Gamma}, \tau)$ with regular section $t_{U}: G \rightarrow \widehat{\Gamma}$. By Proposition 6.5, $\left|\overline{\mathcal{L}}_{U}\right| \cong E$ as bundles over $B G$. In particular, there is a mod $p$ equivalence from $\left|\overline{\mathcal{L}}_{U}\right|$ to $X \times_{G} E G \simeq Y$. 
By Theorem 5.4, there is a saturated fusion system $\mathcal{F}$ over a discrete $p$-toral group $S$, and a transporter system $\mathcal{T}$ associated to $\mathcal{F}$, such that $\overline{\mathcal{L}} \unlhd \mathcal{T}$, $\operatorname{Aut}_{\mathcal{T}}(\bar{S}) / \operatorname{Aut}_{\overline{\mathcal{L}}}(\bar{S}) \cong G$, and such that $|\mathcal{T}|$ contains $\left|\overline{\mathcal{L}}_{U}\right|$ as deformation retract. Hence $|\mathcal{T}|_{p}^{\wedge} \simeq\left|\overline{\mathcal{L}}_{U}\right|_{p}^{\wedge} \simeq Y_{p}^{\wedge}$. Let $\mathcal{T}_{1} \subseteq \mathcal{T}$ be the full subcategory whose objects are the objects of $\mathcal{T}$ which are $\mathcal{F}-$ centric. Then $\mathrm{Ob}\left(\mathcal{T}_{1}\right)$ is invariant under $\mathcal{F}$-conjugacy, closed under overgroups, and contains all subgroups which are $\mathcal{F}$-centric and $\mathcal{F}$-radical. Hence $\left|\mathcal{T}_{1}\right|_{p}^{\wedge} \simeq|\mathcal{T}|_{p}^{\wedge}$ by Proposition A.9(a). By Proposition A.6, there is a linking system $\mathcal{L}_{1}$ associated to $\mathcal{T}_{1}$ and to $\mathcal{F}$, with $\operatorname{Ob}\left(\mathcal{L}_{1}\right)=\operatorname{Ob}\left(\mathcal{T}_{1}\right)$, and such that $\left|\mathcal{L}_{1}\right|_{p}^{\wedge} \simeq\left|\mathcal{T}_{1}\right|_{p}^{\wedge}$. By Proposition A.12, there is a centric linking system $\mathcal{L}$ associated to $\mathcal{F}$ which contains $\mathcal{L}_{1}$ as a full subcategory, and $|\mathcal{L}|_{p}^{\wedge} \simeq\left|\mathcal{L}_{1}\right|_{p}^{\wedge}$ by Corollary A.10. So $|\mathcal{L}|_{p}^{\wedge} \simeq Y_{p}^{\wedge}$, and this finishes the proof of the theorem.

\section{Appendix: Transporter systems over discrete $p$-toral groups}

Transporter systems are a generalization of linking systems, which were first defined (over finite $p$-groups) in [17]. We need them here in order to state our main theorem on extensions of fusion and linking systems (Theorem 5.4) in sufficient generality. In the first half of this section, we define and prove the basic properties of transporter systems over discrete $p$-toral groups; especially those properties needed to prove and apply Theorem 5.4.

Afterwards, we give some conditions under which an inclusion of transporter or linking systems (one a full subcategory of the other) induces an equivalence or $\bmod p$ equivalence of geometric realizations. We also prove that every linking system all of whose objects are centric can be embedded in a centric linking system. When it is a question of adding only finitely many conjugacy classes, these results can be proven using arguments similar to those already used in [5; 17] for linking and transporter systems over finite $p$-groups. What is new here (and makes the proofs harder) is the necessity of handling infinitely many classes at a time.

Let $G$ be a group, and let $\mathcal{H}$ be a family of subgroups of $G$ which is invariant under $G$-conjugacy and overgroups. The transporter system of $G$ with respect to $\mathcal{H}$ is the category $\mathcal{T}_{\mathcal{H}}(G)$ with object set $\mathcal{H}$, and which has morphism sets

$$
\operatorname{Mor}_{\mathcal{T}_{\mathcal{H}}(G)}(P, Q)=T_{G}(P, Q):=\left\{x \in G \mid x P x^{-1} \leq Q\right\}
$$

(the transporter set) for each pair of subgroups $P, Q \in \mathcal{H}$.

Definition A.1 Let $\mathcal{F}$ be a fusion system over a discrete $p$-toral group $S$. A transporter system associated to a fusion system $\mathcal{F}$ is a nonempty category $\mathcal{T}$ such that 
$\mathrm{Ob}(\mathcal{T}) \subseteq \mathrm{Ob}(\mathcal{F})$ is closed under $\mathcal{F}$-conjugacy and overgroups, together with a pair of functors

$$
\mathcal{T}_{\mathrm{Ob}(\mathcal{T})}(S) \stackrel{\varepsilon}{\longrightarrow} \mathcal{T} \stackrel{\rho}{\longrightarrow} \mathcal{F},
$$

satisfying the following conditions.

(A1) The functor $\varepsilon$ is the identity on objects and the functor $\rho$ is the inclusion on objects.

(A2) For each $P, Q \in \mathrm{Ob}(\mathcal{T})$, the kernel

$$
E(P):=\operatorname{Ker}\left[\rho_{P}: \operatorname{Aut}_{\mathcal{T}}(P) \longrightarrow \operatorname{Aut}_{\mathcal{F}}(P)\right]
$$

acts freely on $\operatorname{Mor}_{\mathcal{T}}(P, Q)$ by right composition, and $\rho P, Q: \operatorname{Mor}_{\mathcal{T}}(P, Q) \rightarrow$ $\operatorname{Hom}_{\mathcal{F}}(P, Q)$ is the orbit map for this action. Also, $E(Q)$ acts freely on the set $\operatorname{Mor}_{\mathcal{T}}(P, Q)$ by left composition.

(B) For each $P, Q \in \mathrm{Ob}(\mathcal{T}), \varepsilon_{P}, Q: N_{S}(P, Q) \rightarrow \operatorname{Mor}_{\mathcal{T}}(P, Q)$ is injective, and the composite $\rho_{P, Q} \circ \varepsilon_{P, Q}$ sends $g \in N_{S}(P, Q)$ to $c_{g} \in \operatorname{Hom}_{\mathcal{F}}(P, Q)$.

(C) For all $\varphi \in \operatorname{Mor}_{\mathcal{T}}(P, Q)$ and all $g \in P$, the diagram

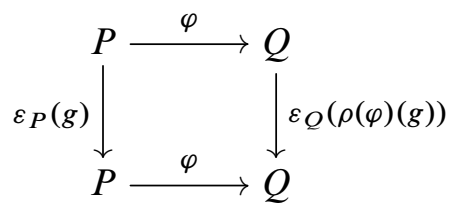

commutes in $\mathcal{T}$.

(I) Each $\mathcal{F}$-conjugacy class of subgroups in $\mathrm{Ob}(\mathcal{T})$ contains a subgroup $P$ such that $\varepsilon_{P}\left(N_{S}(P)\right) \in \operatorname{Syl}_{p}\left(\operatorname{Aut}_{\mathcal{T}}(P)\right)$; ie such that $\left[\operatorname{Aut}_{\mathcal{T}}(P): \varepsilon_{P}\left(N_{S}(P)\right)\right]$ is finite and prime to $p$.

(II) Let $\varphi \in \operatorname{Iso}_{\mathcal{T}}(P, Q), P \unlhd \bar{P} \leq S$, and $Q \unlhd \bar{Q} \leq S$ be such that $\varphi \circ \varepsilon_{P}(\bar{P}) \circ \varphi^{-1} \leq$

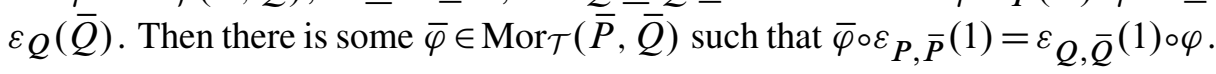

(III) Assume $P_{1} \leq P_{2} \leq P_{3} \leq \cdots$ in $\mathrm{Ob}(\mathcal{T})$ and $\psi_{i} \in \operatorname{Mor}_{\mathcal{T}}\left(P_{i}, S\right)$ are such that for all $i \geq 1, \psi_{i}=\psi_{i+1} \circ \varepsilon_{P_{i}, P_{i+1}}(1)$. Set $P=\bigcup_{i=1}^{\infty} P_{i}$. Then there is $\psi \in \operatorname{Mor}_{\mathcal{T}}(P, S)$ such that $\psi \circ \varepsilon_{P_{i}, P}(1)=\psi_{i}$ for each $i$.

When $P \leq Q$ are both in $\operatorname{Ob}(\mathcal{T})$, we write $\iota_{P, Q}=\varepsilon_{P}, Q(1)$, considered to be the "inclusion" of $P$ into $Q$. By axiom (B), $\rho$ sends $\iota_{P}, Q \in \operatorname{Mor}_{\mathcal{T}}(P, Q)$ to incl ${ }_{P}^{Q} \in$ $\operatorname{Hom}_{\mathcal{F}}(P, Q)$ (the inclusion in the usual sense).

Proposition A.2 The following hold for any transporter system $\mathcal{T}$ associated to a fusion system $\mathcal{F}$ over a discrete $p$-toral group $S$. 
(a) Fix morphisms $\varphi \in \operatorname{Hom}_{\mathcal{F}}(P, Q)$ and $\psi \in \operatorname{Hom}_{\mathcal{F}}(Q, R)$, where $P, Q, R \in$ $\mathrm{Ob}(\mathcal{T})$. Then for any pair of liftings

$$
\tilde{\psi} \in \rho_{Q, R}^{-1}(\psi) \text { and } \widetilde{\psi \varphi} \in \rho_{P, R}^{-1}(\psi \varphi),
$$

there is a unique lifting $\tilde{\varphi} \in \rho_{P, Q}^{-1}(\varphi)$ such that $\tilde{\psi} \circ \tilde{\varphi}=\widetilde{\psi} \varphi$.

(b) For every morphism $\psi \in \operatorname{Mor}_{\mathcal{T}}(P, Q)$, and every $P_{*}, Q_{*} \in \operatorname{Ob}(\mathcal{T})$ such that $P_{*} \leq P, Q_{*} \leq Q$, and $\rho(\psi)\left(P_{*}\right) \leq Q_{*}$, there is a unique morphism $\psi_{*} \in$ $\operatorname{Mor}_{\mathcal{T}}\left(P_{*}, Q_{*}\right)$ such that $\psi \circ \iota_{P, P_{*}}=\iota_{Q, Q_{*}} \circ \psi_{*}$.

(c) For each $\psi \in \operatorname{Mor}(\mathcal{T}), \psi$ is an isomorphism in $\mathcal{T}$ if and only if $\rho(\psi)$ is an isomorphism in $\mathcal{F}$.

(d) All morphisms in $\mathcal{T}$ are monomorphisms and epimorphisms in the categorical sense.

Proof Point (a) follows from [17, Lemma A.7(a)], and (b) is a special case of (a). All morphisms in $\mathcal{T}$ are monomorphisms by [17, Lemma A.7(b)] and since morphisms in $\mathcal{F}$ are monomorphisms.

If $\psi \in \operatorname{Mor}_{\mathcal{T}}(P, Q)$ is such that $\rho(\psi) \in \operatorname{Iso}_{\mathcal{F}}(P, Q)$, then by (a), there are $\psi^{\prime} \in$ $\operatorname{Mor}_{\mathcal{T}}(Q, P)$ and $\psi^{\prime \prime} \in \operatorname{Mor}_{\mathcal{T}}(P, Q)$ such that $\psi \circ \psi^{\prime}=\operatorname{Id}_{Q}$ and $\psi^{\prime} \circ \psi^{\prime \prime}=\operatorname{Id}_{P}$. Then $\psi=\psi \psi^{\prime} \psi^{\prime \prime}=\psi^{\prime \prime}$ is an isomorphism in $\mathcal{T}$ with $\psi^{\prime}$ as inverse. This proves (c).

It remains to prove that all morphisms in $\mathcal{T}$ are epimorphisms. Fix $\psi \in \operatorname{Mor}_{\mathcal{T}}(P, Q)$ and $\varphi_{1}, \varphi_{2} \in \operatorname{Mor}_{\mathcal{T}}(Q, R)$ such that $\varphi_{1} \circ \psi=\varphi_{2} \circ \psi$; we must show that $\varphi_{1}=\varphi_{2}$. Since $\psi$ is the composite of an isomorphism followed by an inclusion by (b) and (c), it suffices to prove this when $P \leq Q$ and $\psi=\iota_{P, Q}$ is the inclusion.

Assume we can show that $\rho\left(\varphi_{1}\right)=\rho\left(\varphi_{2}\right)$. By axiom (A2), $\varphi_{2}=\varphi_{1} \circ \alpha$ for some $\alpha \in E(Q)$. Hence

$$
\varphi_{1} \circ \alpha \circ \iota_{P, Q}=\varphi_{2} \circ \iota_{P, Q}=\varphi_{1} \circ \iota_{P, Q},
$$

so $\alpha \circ \iota_{P, Q}=\iota_{P, Q}$ by (a), and $\alpha=\operatorname{Id}_{Q}$ since $E(Q)$ acts freely on $\operatorname{Mor}_{\mathcal{T}}(P, Q)$ (axiom (A2)). Thus $\varphi_{1}=\varphi_{2}$.

To complete the proof, we need to show that $\rho\left(\varphi_{1}\right)=\rho\left(\varphi_{2}\right)$. Assume otherwise, and let $\bar{P}<Q$ be the subgroup of all $g \in Q$ such that $\rho\left(\varphi_{1}\right)(g)=\rho\left(\varphi_{2}\right)(g)$. Then $P<N_{Q}(\bar{P})$ by Lemma 1.12 , and

$$
\left.\rho\left(\varphi_{1}\right)\right|_{N_{Q}(\bar{P})} \neq\left.\rho\left(\varphi_{2}\right)\right|_{N_{Q}(\bar{P})}
$$

by definition of $\bar{P}$. So upon replacing $P$ by $\bar{P}$ and $Q$ by $N_{Q}(\bar{P})$, we can arrange that $P \unlhd Q$. 
Set $P^{\prime}=\rho\left(\varphi_{1}\right)(P)=\rho\left(\varphi_{2}\right)(P)$. By (b), $\varphi_{1} \circ \iota_{P, Q}=\varphi_{2} \circ \iota_{P, Q}$ has a unique restriction $\beta=\left.\varphi_{1}\right|_{P, P^{\prime}}=\left.\varphi_{2}\right|_{P, P^{\prime}} \in \operatorname{Iso}_{\mathcal{T}}\left(P, P^{\prime}\right)$. Fix $x \in Q$, set $y_{i}=\rho\left(\varphi_{i}\right)(x)$, and consider the following two squares for each $i=1,2$ :

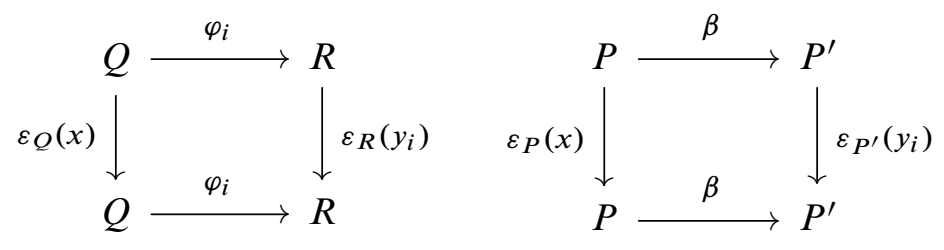

The first square commutes by axiom $(\mathrm{C})$, and the second square is defined to be a restriction of the first. Note that $\varepsilon_{P}(x)$ sends $P$ to itself because $P \unlhd Q^{\prime}$. Note also that $\varepsilon_{P}(x)$ is the restriction of $\varepsilon_{Q^{\prime}}(x)$ since $\varepsilon$ is a functor (and since $\iota_{U, V}=\varepsilon_{U, V}(1)$ for all $U \leq V$ in $\operatorname{Ob}(\mathcal{T}))$. Hence the second square commutes by the uniqueness of restriction morphisms (point (b)). Thus $\varepsilon_{P^{\prime}}\left(y_{1}\right)=\varepsilon_{P^{\prime}}\left(y_{2}\right)=\beta \circ \varepsilon_{P}(x) \circ \beta^{-1}$. Since $\varepsilon_{P^{\prime}}$ is injective (axiom (B)), this shows that $y_{1}=y_{2}$. Since this holds for all $x \in Q, \rho\left(\varphi_{1}\right)=\rho\left(\varphi_{2}\right)$, contradicting our assumption that they are distinct.

In the situation of Proposition A.2(b), we write $\psi_{*}=\left.\psi\right|_{P_{*}, Q_{*}} \in \operatorname{Mor}_{\mathcal{T}}\left(P_{*}, Q_{*}\right)$, and regard it as the restriction of $\psi$.

Proposition A.3 For any fusion system $\mathcal{F}$ over a discrete $p$-toral group $S$, and any transporter system $\mathcal{T}$ associated to $\mathcal{F}, \mathcal{F}$ is $\mathrm{Ob}(\mathcal{T})$-saturated.

Proof Set $\mathcal{H}=\operatorname{Ob}(\mathcal{T})$ for short. By axiom (I) for $\mathcal{T}$, each subgroup in $\mathcal{H}$ is $\mathcal{F}-$ conjugate to some $P$ such that $\varepsilon_{P}\left(N_{S}(P)\right) \in \operatorname{Syl}_{p}\left(\operatorname{Aut}_{\mathcal{T}}(P)\right)$. Hence

$$
\operatorname{Aut}_{S}(P) \in \operatorname{Syl}_{p}\left(\operatorname{Aut}_{\mathcal{F}}(P)\right) \quad \text { and } \quad \varepsilon_{P}\left(C_{S}(P)\right) \in \operatorname{Syl}_{p}(E(P)) \text {, }
$$

where as usual, $E(P)=\operatorname{Ker}\left[\operatorname{Aut}_{\mathcal{T}}(P) \stackrel{\rho_{P}}{\rightarrow} \operatorname{Aut}_{\mathcal{F}}(P)\right]$. In particular, $P$ is fully automized in $\mathcal{F}$.

We claim that $P$ is also receptive. Fix any $Q \in P^{\mathcal{F}}$ and $\varphi \in \operatorname{Iso}_{\mathcal{F}}(Q, P)$. By axiom (A2), there is $\psi \in \operatorname{Iso}_{\mathcal{T}}(Q, P)$ such that $\rho_{Q, P}(\psi)=\varphi$. Let $N_{\varphi} \leq N_{S}(Q)$ be the subgroup of all $g \in N_{S}(Q)$ such that $\varphi c_{g} \varphi^{-1} \in \operatorname{Aut}_{S}(P)$. Then $\psi \varepsilon_{Q}\left(N_{\varphi}\right) \psi^{-1} \leq$ $\varepsilon_{P}\left(N_{S}(P)\right) \cdot E(P)$. Since $\varepsilon_{P}\left(C_{S}(P)\right) \in \operatorname{Syl}_{p}(E(P))$, we have that $\varepsilon_{P}\left(N_{S}(P)\right) \in$ $\operatorname{Syl}_{p}\left(\varepsilon_{P}\left(N_{S}(P)\right) \cdot E(P)\right)$. So there is $\chi \in E(P)$ such that

$$
(\chi \psi) \varepsilon_{Q}\left(N_{\varphi}\right)(\chi \psi)^{-1} \leq \varepsilon_{P}\left(N_{S}(P)\right) .
$$

Axiom (II) now implies that there is $\bar{\psi} \in \operatorname{Mor}_{\mathcal{T}}\left(N_{\varphi}, S\right)$ such that $\bar{\psi} \circ \iota_{Q, N_{\varphi}}=\iota_{P, S} \circ \chi \psi$, and so $\rho(\bar{\psi}) \in \operatorname{Hom}_{\mathcal{F}}\left(N_{\varphi}, S\right)$ is an extension of $\varphi=\rho(\psi)=\rho(\chi \psi)$. 
It remains to prove that axiom (III) (for a fusion system) holds for all subgroups in $\mathcal{H}$. So assume $P_{1} \leq P_{2} \leq P_{3} \leq \cdots$ are subgroups in $\mathcal{H}, P=\bigcup_{i=1} P_{i}$, and $\varphi \in \operatorname{Hom}(P, S)$ is such that $\varphi_{i}:=\left.\varphi\right|_{P_{i}} \in \operatorname{Hom}_{\mathcal{F}}\left(P_{i}, S\right)$ for each $i$. For each $i, E\left(P_{i}\right)$ contains a discrete $p$-toral group with index prime to $p$, and thus is an extension of a discrete $p$-torus by a finite group. Also, restriction defines a homomorphism from $E\left(P_{i+1}\right)$ to $E\left(P_{i}\right)$, and this is injective by Proposition A.2(d). We can thus regard the $E\left(P_{i}\right)$ as a decreasing sequence of discrete- $p$-toral-by-finite groups, and any such sequence becomes constant, since discrete $p$-toral groups are Artinian (see [9, Proposition 1.2]). In other words, there is $N$ such that for all $j>i \geq N$, the restriction of $E\left(P_{j}\right)$ to $E\left(P_{i}\right)$ is an isomorphism.

For each $i \leq N$, choose $\psi_{i}^{\prime} \in \operatorname{Hom}_{\mathcal{T}}\left(P_{i}, S\right)$ such that $\varphi_{i}=\rho_{P_{i}, S}\left(\psi_{i}^{\prime}\right)$. Set $\psi_{N}=\psi_{N}^{\prime}$. By (A2), for each $i>N$, there is $\chi_{i} \in E\left(P_{N}\right)$ such that $\psi_{N}=\left.\psi_{i}^{\prime}\right|_{P_{N}, S} \circ \chi_{i}$. We just saw that there is $\bar{\chi}_{i} \in E\left(P_{i}\right)$ such that $\left.\bar{\chi}_{i}\right|_{P_{N}}=\chi_{i}$. So if we set $\psi_{i}=\psi_{i}^{\prime} \circ \bar{\chi}_{i}$, then $\left.\psi_{i}\right|_{P_{N}, S}=\left.\psi_{i}^{\prime}\right|_{P_{N}, S} \circ \chi_{i}=\psi_{N}$. Since morphisms in $\mathcal{T}$ are epimorphisms by Proposition A.2(d), $\left.\psi_{i+1}\right|_{P_{i}, S}=\psi_{i}$ for each $i>N$, and by axiom (III) for $\mathcal{T}$, there is $\psi \in \operatorname{Mor}_{\mathcal{T}}(P, S)$ such that $\left.\psi\right|_{P_{i}, S}=\psi_{i}$ for each $i \geq N$. Then $\left.\rho(\psi)\right|_{P_{i}, S}=\varphi_{i}$ for each $i$, so $\varphi=\rho(\psi) \in \operatorname{Hom}_{\mathcal{F}}(P, S)$.

We next show that linking systems over saturated fusion systems are transporter systems and characterize linking systems among transporter systems. This is a generalization to linking systems over discrete $p$-toral groups of [16, Proposition 4]. We will first list the properties of linking systems that we need as a separate proposition that might be useful for future reference and then state the result as a corollary.

Proposition A.4 The following hold for any linking system $\mathcal{L}$ associated to a saturated fusion system $\mathcal{F}$ over a discrete $p$-toral group $S$.

(a) For each $P, Q \in \operatorname{Ob}(\mathcal{L})$, the subgroup $E(P):=\operatorname{Ker}\left[\operatorname{Aut}_{\mathcal{L}}(P) \rightarrow \operatorname{Aut}_{\mathcal{F}}(P)\right]$ acts freely on $\operatorname{Mor}_{\mathcal{L}}(P, Q)$ via right composition, and $\pi_{P, Q}$ induces a bijection

$$
\operatorname{Mor}_{\mathcal{L}}(P, Q) / E(P) \stackrel{\cong}{\longrightarrow} \operatorname{Hom}_{\mathcal{F}}(P, Q) .
$$

(b) The functor $\delta$ is injective on all morphism sets.

(c) The action of $E(Q)$ on $\operatorname{Hom}_{\mathcal{F}}(P, Q)$ via left composition is free.

(d) For every morphism $\psi \in \operatorname{Mor}_{\mathcal{L}}(P, Q)$, and every $P_{*}, Q_{*} \in \operatorname{Ob}(\mathcal{L})$ such that $P_{*} \leq P, Q_{*} \leq Q$, and $\pi(\psi)\left(P_{*}\right) \leq Q_{*}$, there is a unique morphism $\left.\psi\right|_{P_{*}, Q_{*}} \in$ $\operatorname{Mor}_{\mathcal{L}}\left(P_{*}, Q_{*}\right)$ (the "restriction" of $\psi$ ) such that $\psi \circ \iota^{P_{*}, P}=\iota^{\imath} Q_{*},\left.Q \circ \psi\right|_{P_{*}, Q_{*}}$.

(e) If $P \in \operatorname{Ob}(\mathcal{L})$ is fully normalized in $\mathcal{F}$, then $\delta_{P}\left(N_{S}(P)\right) \in \operatorname{Syl}_{p}\left(\operatorname{Aut}_{\mathcal{L}}(P)\right)$. 
(f) Let $P \unlhd \bar{P} \leq S$ and $Q \leq \bar{Q} \leq S$ be objects in $\mathcal{L}$. Let $\psi \in \operatorname{Mor}_{\mathcal{L}}(P, Q)$ be such that for each $g \in \bar{P}$, there is $h \in \bar{Q}$ satisfying

$$
\iota^{\imath}, \bar{Q} \circ \psi \circ \delta_{P}(g)=\delta_{Q, \bar{Q}}(h) \circ \psi .
$$

Then there is a unique morphism $\bar{\psi} \in \operatorname{Mor}_{\mathcal{L}}(\bar{P}, \bar{Q})$ such that $\left.\bar{\psi}\right|_{P, Q}=\psi$.

(g) All morphisms in $\mathcal{L}$ are monomorphisms and epimorphisms in the categorical sense.

(h) Assume $P_{1} \leq P_{2} \leq P_{3} \leq \ldots$ in $\operatorname{Ob}(\mathcal{L})$ and $\psi_{i} \in \operatorname{Mor}_{\mathcal{L}}\left(P_{i}, S\right)$ are such that for all $i \geq 1, \psi_{i}=\left.\psi_{i+1}\right|_{P_{i}, S}$. Set $P=\bigcup_{i=1}^{\infty} P_{i}$. Then there is $\psi \in \operatorname{Mor}_{\mathcal{L}}(P, S)$ such that $\left.\psi\right|_{P_{i}, S}=\psi_{i}$ for each $i$.

Proof Points (a) and (b) are exactly the same as points (a) and (c), respectively, in [16, Proposition 4], and the proofs go through unchanged. The proof of (c) is contained in that of [16, Proposition 4(f)], again with no modification necessary. We prove the remaining points.

(d) This is a special case of [9, Lemma 4.3(a)] (which is Proposition A.2(a) for linking systems).

(e) For each $P \in \mathrm{Ob}(\mathcal{L})$ which is fully centralized in $\mathcal{F}$,

$$
\operatorname{Aut}_{\mathcal{F}}(P) \cong \operatorname{Aut}_{\mathcal{L}}(P) / \delta_{P}\left(C_{S}(P)\right) \text { and } \operatorname{Aut}_{S}(P) \cong N_{S}(P) / C_{S}(P) .
$$

Hence $\left[\operatorname{Aut}_{\mathcal{F}}(P): \operatorname{Aut}_{S}(P)\right]=\left[\operatorname{Aut}_{\mathcal{L}}(P): \delta_{P}\left(N_{S}(P)\right)\right]$, since $\delta_{P}$ is injective. If $P \in \mathrm{Ob}(\mathcal{L})$ is fully normalized in $\mathcal{F}$, then $\operatorname{Aut}_{S}(P) \in \operatorname{Syl}_{p}\left(\operatorname{Aut}_{\mathcal{F}}(P)\right)$, and so $\delta_{P}\left(N_{S}(P)\right) \in \operatorname{Syl}_{p}\left(\operatorname{Aut}_{\mathcal{L}}(P)\right)$.

(f) The proof of existence of an extension $\bar{\psi}$ is identical in our case to the proof of the corresponding statement [16, Propostion 4(e)]. It remains to prove uniqueness.

Assume $\bar{\psi}_{1}, \bar{\psi}_{2} \in \operatorname{Iso}_{\mathcal{L}}(\bar{P}, \bar{Q})$ are two extensions of $\psi$. We must show that $\bar{\psi}_{1}=\bar{\psi}_{2}$. It suffices to do this when $\psi$ is an isomorphism, and also (after composing by an isomorphism, if necessary) when $\bar{P}$ is fully centralized in $\mathcal{F}$.

Fix $x \in \bar{P}$, set $y_{i}=\pi\left(\bar{\psi}_{i}\right)(x)$, and consider the following two squares of morphisms in $\mathcal{L}$ :

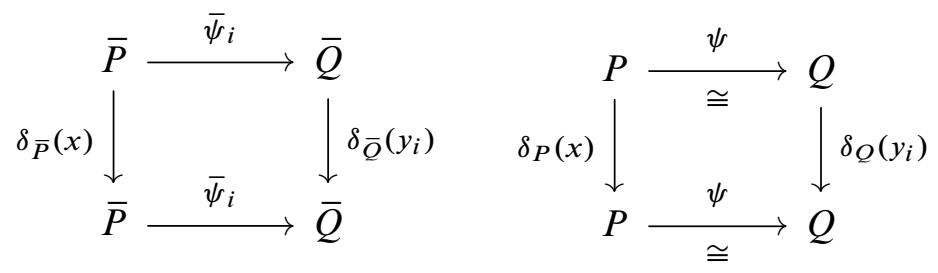


The first square commutes by axiom $(\mathrm{C})$, and the second square is defined to be a restriction of the first. Note that $\delta_{P}(x)$ is the restriction of $\delta_{\bar{P}}(x)$ since $\delta$ is a functor (and since $\iota_{U, V}=\delta_{U, V}(1)$ for all $U \leq V$ in $\operatorname{Ob}(\mathcal{L})$ ). Hence the second square commutes by the uniqueness of restriction morphisms (point (d)). Thus $\delta_{Q}\left(y_{1}\right)=$ $\psi \circ \delta_{P}(x) \circ \psi^{-1}=\delta_{Q}\left(y_{2}\right)$. Since $\delta_{Q}$ is injective by (b), this shows that $y_{1}=y_{2}$. Since this holds for all $x \in \bar{P}, \pi\left(\bar{\psi}_{1}\right)=\pi\left(\bar{\psi}_{2}\right)$.

By axiom (A) (and since $\bar{P}$ is fully centralized), $\bar{\psi}_{2}=\bar{\psi}_{1} \circ \delta_{\bar{P}}(a)$ for some $a \in C_{S}(\bar{P})$. Hence

$$
\begin{aligned}
{ }^{\iota} Q, \bar{Q}^{\circ} \circ=\left.\bar{\psi}_{2}\right|_{P, \bar{Q}}=\left.\left(\bar{\psi}_{1} \circ \delta_{\bar{P}}(a)\right)\right|_{P, \bar{Q}} & =\bar{\psi}_{1} \circ \delta_{P, \bar{P}}(a) \\
& =\left.\bar{\psi}_{1}\right|_{P, \bar{Q}} \circ \delta_{P}(a)={ }^{\iota} Q, \bar{Q} \circ \psi \circ \delta_{P}(a),
\end{aligned}
$$

so $\delta_{P}(a)=1$ since $E(P)$ acts freely on $\operatorname{Mor}_{\mathcal{L}}(P, \bar{Q})$ by (a) (note that $a \in C_{S}(P)$ ). Then $a=1$ since $\delta_{P}$ is injective by (b), and so $\bar{\psi}_{1}=\bar{\psi}_{2}$.

(g) By [17, Lemma A.6], for each $\varphi \in \operatorname{Mor}(\mathcal{L}), \varphi$ is an isomorphism in $\mathcal{L}$ if and only if $\pi(\varphi)$ is an isomorphism in $\mathcal{F}$. Hence by (d), each morphism in $\mathcal{L}$ is the composite of an isomorphism followed by an inclusion. So it suffices to prove that inclusions are monomorphisms and epimorphisms. That they are monomorphisms follows from the uniqueness of restrictions in (d).

By the uniqueness of extensions in (f), any inclusion $\iota_{P, Q}$ for $P \unlhd Q$ is an epimorphism. Since a composite of epimorphisms is an epimorphism, this proves that $\iota_{P, Q}$ is an epimorphism if $P<Q$ with finite index.

Assume this is not true in general. Then there are $\psi_{1}, \psi_{2} \in \operatorname{Iso}_{\mathcal{L}}(P, Q)$ and $P_{*}<P$ such that $\psi_{1} \neq \psi_{2}$ and $\left.\psi_{1}\right|_{P_{*}}=\left.\psi_{2}\right|_{P_{*}}$. Choose $P_{*}<P_{1} \leq P$ which is minimal among subgroups of $P$ containing $P_{*}$ for which $\left.\psi_{1}\right|_{P_{1}} \neq\left.\psi_{2}\right|_{P_{1}}$ ( $S$ is Artinian by [9, Proposition 1.2]). There is no proper subgroup $P_{2}<P_{1}$ of finite index which contains $P_{*}$ (otherwise $\left.\psi_{1}\right|_{P_{2}}=\left.\psi_{2}\right|_{P_{2}}$ and the result follows from (f)), so $P_{1}$ is the union of its proper subgroups which contain $P_{*}$. In particular, $\left.\pi\left(\psi_{1}\right)\right|_{P_{1}}=\left.\pi\left(\psi_{2}\right)\right|_{P_{1}}$. The rest of the argument to show that $\left.\psi_{1}\right|_{P_{1}}=\left.\psi_{2}\right|_{P_{1}}$ (thus giving a contradiction) goes through exactly as in the last paragraph of the proof of (f). (Note that we can easily arrange for $P$ to be fully centralized.)

(h) Assume $P_{1} \leq P_{2} \leq P_{3} \leq \cdots$ in $\operatorname{Ob}(\mathcal{L})$ and $\psi_{i} \in \operatorname{Mor}_{\mathcal{L}}\left(P_{i}, S\right)$ are such that for each $i \geq 1, \psi_{i}=\psi_{i+1} \mid P_{i}, S$. Set $P=\bigcup_{i=1}^{\infty} P_{i}$, and set $\varphi_{i}=\pi\left(\psi_{i}\right) \in \operatorname{Hom}_{\mathcal{F}}\left(P_{i}, S\right)$ for each $i$. Then $\left.\varphi_{i+1}\right|_{P_{i}}=\varphi_{i}$ for each $i$, so the union of the $\varphi_{i}$ is a homomorphism $\varphi \in \operatorname{Hom}(P, S)$. By axiom (III) in Definition 1.4, $\varphi \in \operatorname{Hom}_{\mathcal{F}}(P, S)$.

By Lemma 3.2(d), there is $N$ such that $P_{i}^{\bullet}=P^{\bullet}$ for each $i \geq N$. Let $Q_{N} \in\left(P_{N}\right)^{\mathcal{F}}$ be fully centralized in $\mathcal{F}$, fix $v \in \operatorname{Iso}_{\mathcal{F}}\left(P_{N}, Q_{N}\right)$, and set $Q=v^{\bullet}(P)$. Thus $E\left(Q_{N}\right)=$ 
$\delta_{Q_{N}}\left(C_{S}\left(Q_{N}\right)\right)$, each element of $C_{S}\left(Q_{N}\right)$ also centralizes $Q_{N}^{\bullet}$ by the uniqueness of extensions to $Q_{N}^{\bullet}$, so each element of $E\left(Q_{N}\right)$ extends to an element of $E(Q)$, which implies that each element of $E\left(P_{N}\right)$ extends to an element of $E(P)$.

Choose $\psi^{\prime} \in \operatorname{Mor}_{\mathcal{L}}(P, S)$ such that $\pi\left(\psi^{\prime}\right)=\varphi$. By axiom (A), there is $\chi_{N} \in E\left(P_{N}\right)$ such that $\psi_{N}=\left.\psi^{\prime}\right|_{P_{N}, S} \circ \chi_{N}$. Let $\chi \in E(P)$ be such that $\left.\chi\right|_{P_{N}}=\chi_{N}$, and set $\psi=\psi^{\prime} \circ \chi$. Then $\left.\psi\right|_{P_{N}, S}=\left.\psi^{\prime}\right|_{P_{N}, S} \circ \chi_{N}=\psi_{N}$, and $\left.\psi\right|_{P_{i}, S}=\psi_{i}$ for each $i$ : via composition with inclusions when $i \leq N$, and by (g) (morphisms in $\mathcal{L}$ are epimorphisms) when $i>N$.

We are now ready to show that all linking systems are transporter systems.

Corollary A.5 Fix a saturated fusion system $\mathcal{F}$ over a discrete $p$-toral group $S$. Then each linking system $\mathcal{L}$ associated to $\mathcal{F}$ is also a transporter system. Conversely, a transporter system $\mathcal{T}$ associated to $\mathcal{F}$ which contains all $\mathcal{F}$-centric $\mathcal{F}$-radical subgroups as objects, and such that the kernel subgroups $E(P)$ are all discrete $p$-toral groups, is a linking system.

Proof Assume $\mathcal{L}$ is a linking system associated to $\mathcal{F}$. Axiom (A2) in Definition A.1 follows from Proposition A.4(a),(c), (B) and (C) follow from the corresponding axioms in Definition 1.9, and (I), (II), and (III) follow from points (e), (f), and (h), respectively, in Proposition A.4. Thus $\mathcal{L}$ is a transporter system.

Assume now that $\mathcal{T}$ is a transporter system associated to $\mathcal{F}$ such that $\operatorname{Ob}(\mathcal{T})$ contains all $\mathcal{F}$-centric $\mathcal{F}$-radical subgroups of $S$. By axiom (A2), $\rho_{P, Q}$ sends $\operatorname{Mor}_{\mathcal{T}}(P, Q)$ surjectively onto $\operatorname{Hom}_{\mathcal{F}}(P, Q)$ for each $P, Q \in \mathrm{Ob}(\mathcal{T})$, so every object of $\mathcal{T}$ is isomorphic in $\mathcal{T}$ to an object which is fully centralized. If $P$ is fully centralized, then $\varepsilon_{P}$ sends $C_{S}(P)$ injectively to a Sylow $p$-subgroup of $E(P)$, and hence $E(P)=$ $\varepsilon_{P}\left(C_{S}(P)\right)$ in that case since we are assuming that $E(P)$ is discrete $p$-toral. It follows that $\mathcal{T}$ is a linking system associated to $\mathcal{F}$.

Proposition A.6 Let $\mathcal{F}$ be a saturated fusion system over a discrete $p$-toral group $S$. Let $\mathcal{T}$ be a transporter system associated to $\mathcal{F}$, all of whose objects are $\mathcal{F}$-centric, which contains all $\mathcal{F}$-centric $\mathcal{F}$-radical subgroups of $S$. Then for every $P \in \mathrm{Ob}(\mathcal{T})$, $E(P) \cong E_{0}(P) \times Z(P)$ for some $E_{0}(P) \unlhd E(P)$ which is finite of order prime to $p$ and normal in $\operatorname{Aut}_{\mathcal{T}}(P)$. There is a linking system $\mathcal{L}$ associated to $\mathcal{F}$, defined by setting $\operatorname{Ob}(\mathcal{L})=\operatorname{Ob}(\mathcal{T})$ and

$$
\operatorname{Mor}_{\mathcal{L}}(P, Q)=\operatorname{Mor}_{\mathcal{T}}(P, Q) / E_{0}(P),
$$

and the natural functor $\mathcal{T} \rightarrow \mathcal{L}$ induces a mod $p$ equivalence between the geometric realizations and hence a homotopy equivalence $|\mathcal{T}|_{p}^{\wedge} \simeq|\mathcal{L}|_{p}^{\wedge}$. 
Proof By axiom $(\mathrm{C})$, for all $P \in \mathrm{Ob}(\mathcal{T}), E(P)$ commutes with $\varepsilon_{P}(P)$ in the group $\operatorname{Aut}_{\mathcal{T}}(P)$. Hence $\varepsilon_{P}(Z(P))=\varepsilon_{P}(P) \cap E(P)$ is central in $E(P)$, and it has finiteindex prime to $p$ by axiom (I) and since $P$ is $\mathcal{F}$-centric. So $E(P) \cong Z(P) \times E_{0}(P)$ for some unique $E_{0}(P) \unlhd E(P)$ which is finite of order prime to $p$.

It is now straightforward to check that $\mathcal{L}$, when defined as above, is a quotient category of $\mathcal{T}$ (ie composition is well defined). Axioms (A1), (A2), (B) and (C) for a transporter system imply that $\mathcal{L}$ satisfies the corresponding axioms for a linking system, and thus is a linking system associated to $\mathcal{F}$. The induced map of spaces $|\mathcal{T}| \rightarrow|\mathcal{L}|$ is a mod $p$ equivalence (and hence $|\mathcal{T}|_{p}^{\wedge} \simeq|\mathcal{L}|_{p}^{\wedge}$ ) by the authors [7, Lemma 1.3].

We next define normal transporter subsystems. Recall that for any fusion system $\mathcal{F}$ over a discrete $p$-toral group $S$, a normal subgroup $\bar{S} \unlhd S$ is weakly closed in $\mathcal{F}$ if for each $g \in \bar{S}, g^{\mathcal{F}} \subseteq \bar{S}$. In other words, no element of $\bar{S}$ is $\mathcal{F}$-conjugate to any element of $S \backslash \bar{S}$.

Definition A.7 Fix a pair of saturated fusion systems $\overline{\mathcal{F}} \subseteq \mathcal{F}$ over discrete $p$-toral groups $\bar{S} \unlhd S$, and let $\overline{\mathcal{T}} \subseteq \mathcal{T}$ be associated transporter systems. Then $\overline{\mathcal{T}}$ is normal in $\mathcal{T}(\overline{\mathcal{T}} \unlhd \mathcal{T})$ if:

(i) $\bar{S}$ is strongly closed in $\mathcal{F}$ and $\mathrm{Ob}(\overline{\mathcal{T}})=\{P \cap \bar{S} \mid P \in \mathrm{Ob}(\mathcal{T})\}$.

(ii) For all $P, Q \in \operatorname{Ob}(\overline{\mathcal{T}})$ and $\psi \in \operatorname{Mor}_{\mathcal{T}}(P, Q)$, there are morphisms $\gamma \in \operatorname{Aut}_{\mathcal{T}}(\bar{S})$ and $\psi_{*} \in \operatorname{Mor}_{\overline{\mathcal{T}}}(\gamma(P), Q)$ such that $\psi=\left.\psi_{*} \circ \gamma\right|_{P, \gamma(P)}$.

(iii) For all $P, Q \in \operatorname{Ob}(\overline{\mathcal{T}}), \psi \in \operatorname{Mor}_{\overline{\mathcal{T}}}(P, Q)$, and $\gamma \in \operatorname{Aut}_{\mathcal{T}}(\bar{S}),\left.\gamma\right|_{Q, \gamma}(Q) \circ \psi \circ$ $\left.\gamma\right|_{P, \gamma(P)} ^{-1}$ is in $\operatorname{Mor}_{\overline{\mathcal{T}}}(\gamma(P), \gamma(Q))$.

Here, in (ii) and (iii), we write $\gamma(P)=\rho(\gamma)(P)$ and $\gamma(Q)=\rho(\gamma)(Q)$ for short. In this situation, we define

$$
\mathcal{T} / \overline{\mathcal{T}}=\operatorname{Aut}_{\mathcal{T}}(\bar{S}) / \operatorname{Aut}_{\overline{\mathcal{T}}}(\bar{S})
$$

Let $\mathcal{T}$ be a transporter system. A subgroup $P \in \mathrm{Ob}(\mathcal{T})$ is defined to be $\mathcal{T}$-radical if $O_{p}\left(\operatorname{Aut}_{\mathcal{T}}(P)\right)=\varepsilon_{P}(P)$. When $\mathcal{T}$ is a linking system associated to $\mathcal{F}$, then $P$ is $\mathcal{T}$-radical if and only if it is $\mathcal{F}$-centric and $\mathcal{F}$-radical. If $\mathcal{T}=\mathcal{T}_{S}(G)$ for a finite group $G$ and $S \in \operatorname{Syl}_{p}(G)$, then $P \leq S$ is $\mathcal{T}$-radical if and only if it is a radical $p$-subgroup of $G$ in the usual sense.

Lemma A.8 Let $\mathcal{F}$ be a saturated fusion system over a discrete $p$-toral group $S$, and let $\mathcal{T}$ be a transporter system associated to $\mathcal{F}$. Let $P \in \mathrm{Ob}(\mathcal{T})$ be a minimal object, let $\mathcal{P}$ be the $\mathcal{F}$-conjugacy class of $P$, and let $\mathcal{T}_{0} \subseteq \mathcal{T}$ the full subcategory with object set $\mathrm{Ob}(\mathcal{T}) \backslash \mathcal{P}$. Assume that: 
- $P$ is fully normalized.

- $N_{S}(P) / P$ is finite.

- $\quad P$ is either not $\mathcal{F}$-centric or not $\mathcal{T}$-radical.

Then the inclusion of $\left|\mathcal{T}_{0}\right|$ into $|\mathcal{T}|$ induces an isomorphism in mod $p$ cohomology.

Proof Let $\Phi: \mathcal{T}$ op $\rightarrow \mathbb{Z}_{(p)}-\bmod$ be the functor which sends objects in $\mathcal{P}$ to $\mathbb{F}_{p}$ and other objects to 0 , regarded as a subfunctor of the constant functor $\underline{F}_{p}$. By the minimality of $P$, and since the quotient functor $\underline{\mathbb{F}}_{p} / \Phi$ vanishes on $\mathcal{P}$, it follows that

$$
H^{*}\left(\mathcal{T}, \underline{\mathbb{F}}_{p} / \Phi\right) \cong H^{*}\left(\mathcal{T}_{0}, \underline{\mathbb{F}}_{p}\right) \cong H^{*}\left(\left|\mathcal{T}_{0}\right|, \mathbb{F}_{p}\right)
$$

where the second isomorphism holds because $\mathbb{F}_{p}$ is constant on $\mathcal{T}_{0}$. Upon applying $H^{*}(\mathcal{T},-)$ to the short exact sequence of functors associated to the inclusion of $\Phi$ in $\underline{\mathbb{F}}_{p}$, and using the above isomorphism, we conclude that $H^{*}(\mathcal{T}, \Phi) \cong H^{*}\left(|\mathcal{T}|,\left|\mathcal{T}_{0}\right|, \mathbb{F}_{p}\right)$.

Let $\mathcal{O}(\mathcal{T})$ denote the orbit category associated to $\mathcal{T}$ : the category with the same objects as $\mathcal{T}$, and with morphism sets $\operatorname{Mor}_{\mathcal{O}(\mathcal{T})}(P, Q)=\operatorname{Mor}_{\mathcal{T}}(P, Q) / Q$, where $Q \cong \varepsilon_{Q}(Q)$ acts freely by left composition. The projection $\mathcal{T} \rightarrow \mathcal{O}(\mathcal{T})$ is target regular in the sense of [17, Definition A.5(b)], so by [17, Proposition A.11], there is a spectral sequence

$$
E_{2}^{i j}=H^{i}\left(\mathcal{O}(\mathcal{T}) ; H^{j}(-; \Phi(-))\right) \Longrightarrow H^{i+j}(\mathcal{T} ; \Phi)
$$

Since $N_{S}(P) / P$ is finite, and $\left[\operatorname{Aut}_{\mathcal{T}}(P): \varepsilon_{P}\left(N_{S}(P)\right)\right]<\infty$ by axiom (I) (recall $P$ is fully normalized), $\operatorname{Aut}_{\mathcal{T}}(P) / \varepsilon_{P}(P)$ is also finite. By [9, Proposition 5.4], and since $\Phi(Q)=0$ for $Q \notin \mathcal{P}$,

$$
\begin{aligned}
H^{i}\left(\mathcal{O}(\mathcal{T}) ; H^{j}(-; \Phi(-))\right) & \cong \Lambda^{i}\left(\operatorname{Aut}_{\mathcal{T}}(P) / \varepsilon_{P}(P) ; H^{j}(P ; \Phi(P))\right) \\
& \cong \Lambda^{i}\left(\operatorname{Aut}_{\mathcal{T}}(P) / \varepsilon_{P}(P) ; H^{j}\left(P ; \underline{\mathbb{F}}_{p}\right)\right)
\end{aligned}
$$

for each $i$ and $j$. If $P$ is not $\mathcal{F}$-centric (and since it is fully normalized), there is $g \in C_{S}(P) \backslash P$, and the class of $\varepsilon_{P}(g) \neq 1$ in $\operatorname{Aut}_{\mathcal{T}}(P) / \varepsilon_{P}(P)$ acts trivially on $H^{j}\left(P ; \underline{\mathbb{F}}_{p}\right)$. If $P$ is not $\mathcal{T}$-radical, then $O_{p}\left(\operatorname{Aut}_{\mathcal{T}}(P) / \varepsilon_{P}(P)\right) \neq 1$. In either case, by Jackowski, McClure and the third author [14, Proposition 6.1(ii)], we have that $\Lambda^{i}\left(\operatorname{Aut}_{\mathcal{T}}(P) / \varepsilon_{P}(P) ; H^{j}\left(P ; \underline{\mathbb{F}}_{p}\right)\right)=0$ for each $i$ and $j$, so

$$
H^{*}\left(|\mathcal{T}|,\left|\mathcal{T}_{0}\right| ; \mathbb{F}_{p}\right) \cong H^{*}(\mathcal{T} ; \Phi)=0
$$

Thus the inclusion $\left|\mathcal{T}_{0}\right| \rightarrow|\mathcal{T}|$ induces an isomorphism on mod $p$ cohomology as claimed. 
Lemma A.8 gives us a tool for proving that under certain conditions, adding or subtracting one conjugacy class in a transporter system does not change the ( $\bmod p)$ homotopy type of its realization. However, more is needed when dealing with infinitely many conjugacy classes. One problem when doing this is that the "bullet functor" $P \mapsto P^{\bullet}$ need not lift to a functor on a transporter system. This is why we need to assume the existence of a normal linking subsystem in the following proposition.

Proposition A.9 Let $\mathcal{F}$ be a saturated fusion system over a discrete $p$-toral group $S$, and let $\mathcal{T}$ be a transporter system associated to $\mathcal{F}$. Let $\mathcal{T}_{0} \subseteq \mathcal{T}$ be a full subcategory which is a transporter subsystem associated to a full subcategory $\mathcal{F}_{0} \subseteq \mathcal{F}$. Assume there is a normal linking subsystem $\overline{\mathcal{L}} \unlhd \mathcal{T}$ over a subgroup $\bar{S} \leq S$ of finite index, and let $\overline{\mathcal{F}} \subseteq \mathcal{F}$ be the corresponding fusion subsystem. Assume also that either:

(a) All objects in $\overline{\mathcal{L}}$ are $\overline{\mathcal{F}}$-centric and $\operatorname{Ob}\left(\mathcal{T}_{0}\right) \supseteq \operatorname{Ob}(\mathcal{T}) \cap \mathrm{Ob}\left(\mathcal{F}^{c}\right)$.

(b) Each object in $\operatorname{Ob}(\mathcal{T}) \backslash \mathrm{Ob}\left(\mathcal{T}_{0}\right)$ is $\mathcal{F}$-centric and not $\mathcal{T}$-radical.

Then the inclusion of $\left|\mathcal{T}_{0}\right|$ in $|\mathcal{T}|$ is a mod $p$ homology equivalence.

Proof For each $P \leq S$, we write $\bar{P}=P \cap \bar{S}$, and set $P^{\vee}=P \bar{P}^{\bullet}$ (where (-) means the bullet construction for $\mathcal{F})$. By Lemma 3.2(b), $N_{S}(\bar{P}) \leq N_{S}\left(\bar{P}^{\bullet}\right)$, and since $\bar{P} \unlhd P$, it follows that $P$ normalizes $\bar{P}^{\bullet}$ (so $P^{\vee}$ is a subgroup). Also, since $\bar{P}^{\bullet} \leq \bar{S}$, $\overline{P^{\vee}}=\bar{P} \cdot \bar{P}^{\bullet}=\bar{P}^{\bullet}$, and so $\left(P^{\vee}\right)^{\vee}=P^{\vee}$.

For each $P, Q \in \operatorname{Ob}(\mathcal{T})$ and each $\psi \in \operatorname{Mor}_{\mathcal{T}}(P, Q)$, let $\bar{\psi}=\left.\psi\right|_{\bar{P}}, \bar{Q}$. Then $\bar{\psi}$ extends to $\psi \in \operatorname{Mor}_{\mathcal{T}}(P, Q)$ and to $\bar{\psi}^{\bullet} \in \operatorname{Mor}_{\mathcal{T}}\left(\bar{P}^{\bullet}, \bar{Q}^{\bullet}\right)$ (Lemma 3.5(a)), and hence by axiom (II) to $\psi^{\vee} \in \operatorname{Mor}_{\mathcal{T}}\left(P^{\vee}, Q^{\vee}\right)$. By the uniqueness of these extensions, $\left.\psi^{\vee}\right|_{P}=\psi$, and thus $(-)^{\vee}$ defines an idempotent functor from $\mathcal{T}$ to itself.

Let $\mathcal{T}^{\vee} \subseteq \mathcal{T}$ be the full subcategory whose objects are those $P$ such that $P^{\vee}=P$ (and use the same notation for the corresponding subcategory of any subtransporter system of $\mathcal{T}$ ). For each $P \leq S, P^{\vee} / \bar{P}^{\bullet}$ is a subgroup of order at most $|S / \bar{S}|$ in $N_{S}\left(\bar{P}^{\bullet}\right) / \bar{P}^{\bullet}$, and by [9, Lemma 1.4(a)], there are only finitely many conjugacy classes of such subgroups. Since $\mathcal{F}^{\bullet}$ has finitely many $S$-conjugacy classes of objects, $\mathcal{T}^{\vee}$ also has finitely many $S$-conjugacy classes of objects.

Let $\mathfrak{H}$ be the set of all transporter subsystems $\mathcal{T}^{\prime} \subseteq \mathcal{T}$ such that:

- $\mathcal{T}^{\prime} \supseteq \mathcal{T}_{0}$.

- $\mathcal{T}^{\prime}$ is a full subcategory of $\mathcal{T}$.

- The inclusion $\left|\mathcal{T}_{0}\right| \subseteq\left|\mathcal{T}^{\prime}\right|$ is a mod $p$ homology equivalence in case (a), or a homotopy equivalence in case (b).

We must show that $\mathcal{T} \in \mathfrak{H}$. 
Assume otherwise, and choose $\mathcal{T}_{1} \in \mathfrak{H}$ for which $\mathrm{Ob}\left(\mathcal{T}_{1}\right) \cap \mathrm{Ob}\left(\mathcal{T}^{\vee}\right)$ contains the largest possible number of $\mathcal{F}$-conjugacy classes. Let $\mathcal{T}_{2} \subseteq \mathcal{T}$ be the full subcategory with $\mathrm{Ob}\left(\mathcal{T}_{2}\right)=\left\{P \in \mathrm{Ob}(\mathcal{T}) \mid P^{\vee} \in \mathrm{Ob}\left(\mathcal{T}_{1}\right)\right\}$. By the above discussion, $\left|\mathcal{T}_{1}^{\vee}\right|=\left|\mathcal{T}_{2}^{\vee}\right|$ is a strong deformation retract of $\left|\mathcal{T}_{1}\right|$ and of $\left|\mathcal{T}_{2}\right|$, so $\left|\mathcal{T}_{1}\right| \simeq\left|\mathcal{T}_{2}\right|$, and $\mathcal{T}_{2} \in \mathfrak{H}$.

Since $\mathcal{T}_{2} \varsubsetneqq \mathcal{T}$ by assumption, $\mathrm{Ob}\left(\mathcal{T}^{\vee}\right) \nsubseteq \mathrm{Ob}\left(\mathcal{T}_{2}\right)$. Let $P$ be maximal among objects in $\mathcal{T}^{\vee}$ not in $\mathcal{T}_{2}$. By definition of $\mathrm{Ob}\left(\mathcal{T}_{2}\right), P$ is maximal among all objects of $\mathcal{T}$ not in $\mathcal{T}_{2}$. Let $\mathcal{T}_{3} \subseteq \mathcal{T}$ be the full subcategory with $\mathrm{Ob}\left(\mathcal{T}_{3}\right)=\mathrm{Ob}\left(\mathcal{T}_{2}\right) \cup P^{\mathcal{F}}$.

Now, $N_{S}(P) / P C_{S}(P) \cong \operatorname{Out}_{S}(P)$ is finite by [9, Lemma 2.5]. In case (a), the group $P C_{S}(P) / P$ is finite since $\bar{P}$ is $\overline{\mathcal{F}}$-centric and $[S: \bar{S}]<\infty$, while $C_{S}(P) \leq P$ in case (b). So $\left|N_{S}(P) / P\right|<\infty$ in either case.

By assumption, $P$ is either not $\mathcal{F}$-centric (case (a)) or not $\mathcal{T}$-radical (case (b)). So by Lemma A.8, the inclusion of $\left|\mathcal{T}_{2}\right|$ into $\left|\mathcal{T}_{3}\right|$ is a $\bmod p$ equivalence. Hence $\mathcal{T}_{3} \in \mathfrak{H}$, contradicting our maximality assumption on $\mathcal{T}_{1}$.

Corollary A.10 Let $(S, \mathcal{F}, \mathcal{L})$ be a linking triple, and let $\mathcal{L}_{0} \subseteq \mathcal{L}$ be a full subcategory which is a linking subsystem associated to some $\mathcal{F}_{0} \subseteq \mathcal{F}$. Then the inclusion of $\left|\mathcal{L}_{0}\right|$ into $|\mathcal{L}|$ is a $\bmod p$ homology equivalence.

Proof Since $\mathcal{L}$ is a linking system, a subgroup $P \leq S$ is $\mathcal{L}$-radical exactly when it is $\mathcal{F}$-centric and $\mathcal{F}$-radical. So by definition, $\mathcal{L}_{0}$ contains all $\mathcal{L}$-radical subgroups. Hence the lemma is a special case of Proposition A.9(b).

It remains to look at the problem of constructing a centric linking system which contains as full subcategory a linking system over a smaller set of objects. As usual, we first check what happens when we add one conjugacy class of objects.

Lemma A.11 Let $\mathcal{F}$ be a saturated fusion system over a discrete $p$-toral group $S$, and let $\mathcal{F}^{c}$ be the full subcategory of $\mathcal{F}$-centric objects. Let $\mathcal{F}_{0} \subseteq \mathcal{F}_{1} \subseteq \mathcal{F}^{c}$ be full subcategories such that $\mathrm{Ob}\left(\mathcal{F}_{0}\right)$ and $\mathrm{Ob}\left(\mathcal{F}_{1}\right)$ are invariant under $\mathcal{F}$-conjugacy and closed under overgroups, $\mathcal{F}_{0}$ contains all $\mathcal{F}$-centric $\mathcal{F}$-radical subgroups of $S$, and $\mathrm{Ob}\left(\mathcal{F}_{1}\right)=\mathrm{Ob}\left(\mathcal{F}_{0}\right) \cup \mathcal{P}$ for some $\mathcal{F}$-conjugacy class $\mathcal{P}$. If $\mathcal{L}_{0}$ is a linking system associated to $\mathcal{F}_{0}$, then there is a linking system $\mathcal{L}_{1}$ associated to $\mathcal{F}_{1}$ such that $\mathcal{L}_{0}$ is isomorphic as a linking system to the full subcategory of $\mathcal{L}_{1}$ with same set of objects as $\mathcal{L}_{0}$.

Proof This follows by the same proof as in [2, Proposition III.4.8] (Steps 1-3), together with Lemma 2.4. 
Alternatively, let $\Phi$ be the functor $\Phi(P)=Z(P)$ when $P \in \mathcal{P}$, and $\Phi(P)=0$ when $P \in \mathrm{Ob}\left(\mathcal{F}_{0}\right)$. For $P \in \mathcal{P}, \Lambda^{*}\left(\operatorname{Out}_{\mathcal{F}}(P) ; \Phi(P)\right)=0$ by [14, Proposition 6.1(ii)] and since $O_{p}\left(\operatorname{Out}_{\mathcal{F}}(P)\right) \neq 1$. Hence by $\left[9\right.$, Proposition 5.4], $H^{3}\left(\mathcal{O}\left(\mathcal{F}_{1}\right) ; \Phi\right)=0$. By an argument similar to that used by the authors to prove [8, Proposition 3.1], the obstruction to extending $\mathcal{L}_{0}$ to $\mathcal{L}_{1}$ lies in $H^{3}\left(\mathcal{O}\left(\mathcal{F}_{1}\right) ; \Phi\right)$, and hence $\mathcal{L}_{1}$ does exist.

This is now generalized as follows.

Proposition A.12 Let $\mathcal{F}$ be a saturated fusion system over a discrete $p$-toral group $S$, and let $\mathcal{F}^{c}$ be the full subcategory of $\mathcal{F}$-centric objects. Let $\mathcal{F}_{0} \subseteq \mathcal{F}^{c}$ be a full subcategory such that $\operatorname{Ob}\left(\mathcal{F}_{0}\right)$ is invariant under $\mathcal{F}$-conjugacy and closed under overgroups, and contains all $\mathcal{F}$-centric $\mathcal{F}$-radical subgroups of $S$. If $\mathcal{L}_{0}$ is a linking system associated to $\mathcal{F}_{0}$, then there is a centric linking system $\mathcal{L}$ associated to $\mathcal{F}$ (associated to $\mathcal{F}^{c}$ ) which contains $\mathcal{L}_{0}$ as a linking subsystem.

Proof Let $\mathfrak{H}$ be the set of all families of subgroups $\mathcal{H} \subseteq \mathrm{Ob}\left(\mathcal{F}^{c}\right)$ such that:

- $\mathcal{H} \supseteq \operatorname{Ob}\left(\mathcal{F}_{0}\right)$.

- $\mathcal{H}$ is invariant under $\mathcal{F}$-conjugacy and closed under overgroups.

- There is a linking system with object set $\mathcal{H}$ which contains $\mathcal{L}_{0}$ as a linking subsystem.

We must show that $\mathrm{Ob}\left(\mathcal{F}^{c}\right) \in \mathfrak{H}$.

Choose $\mathcal{H}_{1} \in \mathfrak{H}$ for which $\mathcal{H}_{1} \cap \mathcal{F}^{\bullet}$ contains the largest possible number of $\mathcal{F}$ conjugacy classes. Set $\mathcal{H}_{2}=\left\{P \in \mathrm{Ob}\left(\mathcal{F}^{c}\right) \mid P^{\bullet} \in \mathcal{H}_{1}\right\}$, and let $\mathcal{F}_{1} \subseteq \mathcal{F}_{2} \subseteq \mathcal{F}$ be the full subcategories with $\operatorname{Ob}\left(\mathcal{F}_{i}\right)=\mathcal{H}_{i}$. Let $\mathcal{L}_{2}$ be the pullback of $\mathcal{L}_{1}$ and $\mathcal{F}_{2}$ via the functors

$$
\mathcal{L}_{1} \stackrel{\pi_{1}}{\longrightarrow} \mathcal{F}_{1} \stackrel{(-)^{\bullet}}{\longleftarrow} \mathcal{F}_{2}
$$

Then $\mathcal{L}_{2}$ is a linking system associated to $\mathcal{F}_{2}$ (recall that $Z(P)=Z\left(P^{\bullet}\right)$ ), and hence $\mathcal{H}_{2} \in \mathfrak{H}$.

Assume $\operatorname{Ob}\left(\left(\mathcal{F}^{c}\right)^{\bullet}\right) \backslash \mathcal{H}_{1} \neq \varnothing$, and let $P$ be maximal among subgroups in this set. Then $P$ is maximal among subgroups in $\operatorname{Ob}\left(\mathcal{F}^{c}\right) \backslash \mathcal{H}_{2}$, and so $\mathcal{H}_{2} \cup P^{\mathcal{F}} \in \mathfrak{H}$ by Lemma A.11, and this contradicts the choice of $\mathcal{H}_{1}$. Hence $\operatorname{Ob}\left(\left(\mathcal{F}^{c}\right)^{\bullet}\right) \subseteq \mathcal{H}_{1}$, and so $\mathcal{H}_{2}=\mathrm{Ob}\left(\mathcal{F}^{c}\right) \in \mathfrak{H}$. 


\section{References}

[1] K K S Andersen, B Oliver, J Ventura, Reduced, tame and exotic fusion systems, Proc. Lond. Math. Soc. 105 (2012) 87-152 MR2948790

[2] M Aschbacher, R Kessar, B Oliver, Fusion systems in algebra and topology, London Math. Soc. Lecture Note Series 391, Cambridge Univ. Press (2011) MR2848834

[3] M G Barratt, V K A M Gugenheim, J C Moore, On semisimplicial fibre-bundles, Amer. J. Math. 81 (1959) 639-657 MR0111028

[4] A K Bousfield, D M Kan, Homotopy limits, completions and localizations, Lecture Notes in Math. 304, Springer (1972) MR0365573

[5] C Broto, N Castellana, J Grodal, R Levi, B Oliver, Subgroup families controlling p-local finite groups, Proc. London Math. Soc. 91 (2005) 325-354 MR2167090

[6] C Broto, N Castellana, J Grodal, R Levi, B Oliver, Extensions of p-local finite groups, Trans. Amer. Math. Soc. 359 (2007) 3791-3858 MR2302515

[7] C Broto, R Levi, B Oliver, Homotopy equivalences of p-completed classifying spaces of finite groups, Invent. Math. 151 (2003) 611-664 MR1961340

[8] C Broto, R Levi, B Oliver, The homotopy theory of fusion systems, J. Amer. Math. Soc. 16 (2003) 779-856 MR1992826

[9] C Broto, R Levi, B Oliver, Discrete models for the p-local homotopy theory of compact Lie groups and p-compact groups, Geom. Topol. 11 (2007) 315-427 MR2302494

[10] C Broto, R Levi, B Oliver, The rational cohomology of a p-local compact group, Proc. Amer. Math. Soc. 142 (2014) 1035-1043 MR3148537

[11] E B Curtis, Simplicial homotopy theory, Advances in Math. 6 (1971) 107-209 MR0279808

[12] W G Dwyer, C W Wilkerson, Homotopy fixed-point methods for Lie groups and finite loop spaces, Ann. of Math. 139 (1994) 395-442 MR1274096

[13] P G Goerss, J F Jardine, Simplicial homotopy theory, Progress in Mathematics 174, Birkhäuser, Basel (1999) MR1711612

[14] S Jackowski, J McClure, B Oliver, Homotopy classification of self-maps of BG via G-actions, I, Ann. of Math. 135 (1992) 183-226 MR1147962

[15] F Junod, R Levi, A Libman, Unstable Adams operations on p-local compact groups, Algebr. Geom. Topol. 12 (2012) 49-74 MR2889545

[16] B Oliver, Extensions of linking systems and fusion systems, Trans. Amer. Math. Soc. 362 (2010) 5483-5500 MR2657688

[17] B Oliver, J Ventura, Extensions of linking systems with p-group kernel, Math. Ann. 338 (2007) 983-1043 MR2317758 
[18] K Roberts, S Shpectorov, On the definition of saturated fusion systems, J. Group Theory 12 (2009) 679-687 MR2554760

Departament de Matemàtiques, Universitat Autònoma de Barcelona

E-08193 Bellaterra, Spain

Institute of Mathematics, University of Aberdeen

Fraser Noble Building 138, Aberdeen AB24 3UE, UK

Sorbonne Paris Cité, LAGA, UMR 7539 du CNRS, Université Paris 13

99, Avenue J-B Clément, 93430 Villetaneuse, France

broto@mat.uab.cat, r.levi@abdn.ac.uk, bobol@math.univ-paris13.fr

Received: 13 August 2013 Revised: 27 February 2014 
